ABSTRACT Of COVARIANCE AND RELATIVITY

by

Roger B. Angel, Department of Philosophy, Doctor of Philosophy.

Following the inception of General Relativity, a dialogue ensued between A. Einstein and E. Kretschmann concerning the status of the Principle of General Covariance. Einstein supposed it to have physical content whereas Kretschmann proved that all lawlike statements of physics are expressible in generally covariant form. Einstein finally concurred with Kretschmann. Subsequently, M. Bunge argued that all covariance principles are metatheoretical, i.e. their referents are not physical patterns but theories. However, recently, J.I. Anderson argued that the imposition of covariance requirements restricts the possible forms of the laws of a given theory, indicating that they do have physical content. We show that there is an element of truth in both positions. However, the dispute has rested on a confusion between principles of covariance and principles of relativity, the former being formal and the latter being physical. We distinguish between the covariance group and the relativity group of several theories in support of our contention. 
Title which is to appear on the spine of the bound copy:

RELATIVITY AND COVARIANCE - Roger B. Angel. 


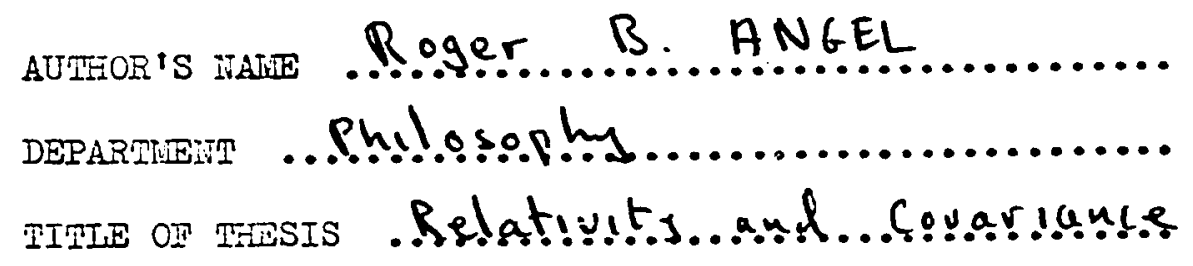

Authorization is iereby given to lickill University to make this thesis available to readers either in a ifoill University Library, or elsewhere, either in its present Iorm or in reproduction. The autior reserves other publication rights, and neither the thesis nor extensive extracts from it nay be printed or otherwise reproauced without the author's written permission.

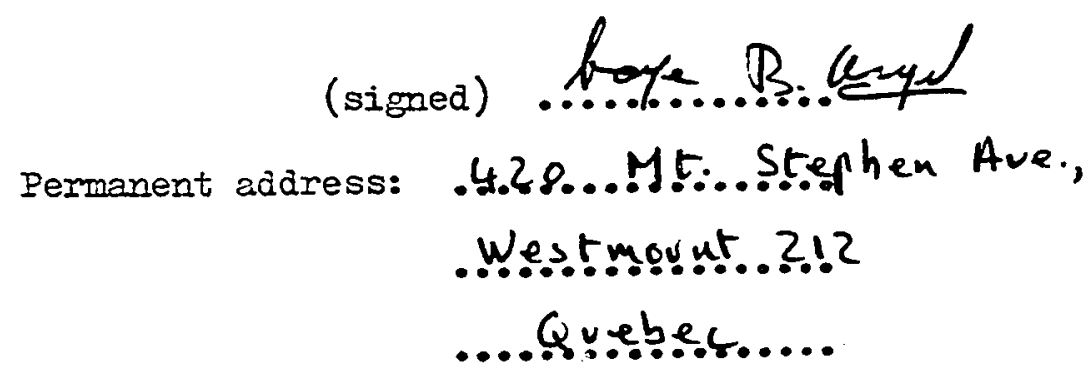

Dated ... November 20.1. 
ABSTRACT Of COVARIANCE AND RELATIVITY

by

Roger B. Angel,

Department of Philosophy,

Doctor of Philosophy.

Following the inception of General Relativity, a dialogue ensued between $A$. Einstein and E. Kretschmann concerning the status of the Principle of General

Covariance. Einstein supposed it to have physical content whereas Kretschmann proved that all lawlike statements of physics are expressible in generally covariant form. Einstein finally concurred with Kretschmann. Subsequently, M. Bunge argued that all covariance principles are metatheoretical, i.e. their referents are not physical patterns but theories. However, recentIy, J.I. . Anderson argued that the imposition of covariance requirements restricts the possible forms of the laws of a given theory, indicating that they do have physical content. We show that there is an element of truth in both positions. However, the dispute has rested on a confusion between principles of covariance and principles of relativity, the former being formal and the latter being physical. We distinguish between the covariance group and the relativity group of several theories in support of our contention. 
Title which is to appear on the spine of the bound copy: RELATIVITY AND COVARIANCE - Roger B. Angel. 


\section{RELATIVITY AND COVARIANCE}

\section{by}

Roger B. Angel, M.A.

A dissertation submitted to the Faculty of Graduate studies and Research in partial fulfilment of the requirements for the degree of Doctor of Philosophy.

Department of Philosophy, McGill University, Montreal. 
to

M. B.

Plato amicus, sed magis amica veritas. 


\section{TABLE OF CONTENTS}

$\begin{array}{ll}\text { PREFACE } & 1\end{array}$

$\begin{array}{lll}\text { CHAPTER I INTRODUCTION } & 6\end{array}$

CHAPTER II TECHNICAL PREIIMINARIES 15

CHAPTER III IAWS AND METAIAWS 46

CHAPTER IV THE PRINCIPLE OF CLASSICAL RELATIVITY 58

CHAPTER V SPECIAL RELATIVITY KINEMATICS 83

CHAPTER VI SPECIAL RELATIVITY MECHANICS 112

CHAPTER VII ELECTRODYNAMICS 131

CHAPTER VIII THE PRINCIPLE OF GENERAI RELATIVITY 163

$\begin{array}{ll}\text { APPENDIX TO CHAPTER VII } & 217\end{array}$

BIBLIOGRAPHY $\quad 222$ 


\section{PREFACE}

The author hopes that this dissertation

on the philosophy of physics will prove to be of interest to both philosophers and physicists. However, in addressing himself to this dual audience, he has been presented with the difficult task of composing a work which would be relatively intelligible to both. In fact, any thoughtful physicist should have little difficulty in appreciating the significance of this work. On the other hand, however, the phiosopher who lacks training in applied mathematics may encounter some difficulty. Accordingly, certain compromises have been made in the mode of presentation. The author has presupposed only a minimal knowledge of physics and applied mathematics on the part of his philosophical reader. All of the more advanced technical apparatus has been explained, although sketchily, as it is needed. Of course, we could not start from zero in a work of this nature. However, we presuppose that the philosopher has onl $\ddot{y}$ a knowledge of the rudiments of the differential and integral calculus, elementary vector analysis including the theorems of Gauss and Stokes, a passing acquaintance with the theory of matrices, an acquaintance with the algebraic concept of a group, and as much physics as 
would be covered in a first year university course. The writer has made an effort to introduce the more advanced topics step by step and as painlessly as possible.

Having been trained in philosophy, but not in mathematical physics, the writer knows how useful this particular form of sympathy can be.

The notation employed is largely that of standard mathematics. In the case of Cartesian vectors and tensors, no distinction is made between the covariant and contravariant components, both of which are represented by superscript indices, with the exception of the metric coefficients which are denoted by subscripts. $\boldsymbol{d}_{\boldsymbol{m}}$ is the Kronecker symbol, and the Einstein summation conventicn is used throughout. In place of the common practice of using primes to denote transformed variables, etc., we have preferred to place a bar over the transformed variable, so that primes and tensor indices do not get confused. This practice also makes for a more tidy appearance. Other notational devices and abbreviations which are less standard are the following:

$\leftrightarrow$ : material equivalence; if and only if; the necessary and sufficient condition.

$=d f$. : equivalent by definition.

=s. : semantically equivalent; having the same meaning; having the same factual content.

$t(1)$ : the transform of 1 by the element ' $t$ ' of a given group of transformations.

w.r.t. : with respect to. 
All other notational conventions are explained as they are introduced.

The primary original contribution of this dissertation is briefly the following. Most, if not all, physical theories are taken to satisfy a principle of relativity of some kind. This property of theories is generally held to be reflected in their mathematical or formal structure which will be such that the fundamental laws of the theory of interest will be covariant under a particular group of coordinate transformations. In general, physicists tend to regard the covariance principle as nothing but the mathematical expression of the relativity principle which is satisfied by the theory. However, this virtual identification of covariance and relativity principles has led to a perplexing problem. The determination of the covariance properties of a theory is a purely formal operation (Bunge, 1961). On the other hand, many physicists would regard the expression of a relativity principle as a statement about physical reality(e.g., Anderson, 1967). Thus, depending on the point of view, principles of relativity are taken to be either metatheoretical statements, devold of physical content, or object statements whose referent is the physical world. Clearly, these points of view are incompatible.

We resolve the difficulty by introducing the notion of the relativity group of a theory as distinct 
from the covariance group of a theory. On the basis of this distinction, we show that covariance principles differ fundamentally from relativity principles. In support of this position we formulate the various principles of relativity in a manner which makes manifest their status as object statements of physical theories. In the course of the argument we endeavour to sharpen the concept of inertial frame of reference and to clarify the distinction between the inertial frames of different theories, although the latter merely supplements the work of our research director, who has already pointed out that the concept of inertial frame varies from one theory to another (Bunge, 1967a). Finally, the work of this dissertation strongly supports the broader moral that an essential prerequisite to the understanding of any physical theory is the recognition of the distinction between the properties of the mathematical formalism which happens to be employed to express that theory and the physical content of the theory, which should be independent of the choice of formal1sm.

The author particularly wishes to acknowledge the profound value of the supervision of Professor Mario Bunge, who made him aware that philosophy is not a gentlemanly game but an earnest quest for knowledge and understanding, which may only be achleved by travelling paths which are both arduous and rigorous. If this 
dissertation has any intellectual merit, it is due solely to his strict guidance and uncompromising desire for truth. 


\section{CHAPTER I}

\section{INTRODUCTION}

An interesting dispute has been simmering in the philosophy of physics for the last fifty years. Recently, fresh attempts have been made to settle it. In his founding pap?r, Einstein(1916) gave the clear impression that the Principle of General Relativity led to new physics. Briefly, he argued that if we adopt this principle on independent epistemological grounds and require a field theory of gravitation to satisfy it, the resulting field equations will entail physical consequences which are significantly different from those of the classical theory of gravitation. The most striking example, perhaps, of the fecundity of this principle was the direct prediction of the precession of the perihelion of Mercury. Thus, it seemed clear that the Principle of General Relativity must have physical content, on the formal ground that in conjunction with certain physical assumptions it entails factual consequences which are not entalled by those assumptions alone.

However, shortly after the publication of Einstein's paper, an article appeared by Kretschmann(1917) which convincingly demonstrated that any putative law, e.g. Newton's laws of motion, could be formulated in a 
generally covariant way, thus satisfying the Principle of General Relativity. Einstein(1918) concurred with the view of Kretschmann, and since that time the bulk of orthodox opinion has followed a similar line.

M. Bunge(e.g., 1961) has frequently argued in favour of the same view on independent logical grounds. Briefly, Bunge's position is to the effect that it is essential to distinguish between the axioms of a theory, e.g. its basic physical hypotheses, and those other lawlike principles which are to be associated with a theory but which actually belong to the corresponding metatheory. In particular, there are principles which he calls laws of physical laws or metalaws whose referents are not the objective patterns of nature but the lawlike descriptions of them. In short, metalaws are not statements about the world but statements about physical laws. According to Bunge, the Principle of General Covariance and, in fact, all covariance principles(Galilei, Lorentz, canonical, etc.) clearly belong to the class of metalaws. on this view, it would be patently absurd to assert that physical reality is generally covariant. Clearly, one should and would say that the basic laws of this or that theory are generally covariant.

A position diametrically opposed to that of Bunge has recently recelved an exposition by J. I. Anderson (1967) in a text-book on relativity physics. Anderson 
argues in considerable detail that the imposition of a covariance requirement(or of a related symmetry requirement) has definite physical consequences. Specifically, he claims to show that when a theoretical description of a physical system is required to admit a specific group of transformations, the choice of basic laws is severely delimited and, in certain cases, may even be uniquely determined.

Anderson disposes of Kretschmann's argument by toeing an empiricist line that any term which occurs in a physical formula must allow of a specific physical and observational interpretation. He argues that Kretschmann was able to render any arbitrary lawlike formula generally covariant by importing terms which model no aspect of the world. In other words, his criticism amounts to the accusation that Kretschmann's argument depended on an ingenious but irrelevant 'cooking of the results.' It is clear that the treatments of Bunge and Anderson have much wider significance than those of Einstein and Kretschmann. Whereas the latter were concerned specifically with the status of general covariance, the former are directed at all covariance principles. Thus, what they have to say has implications for Classical physics and Special Relativity physics as much as for the Theory of General Relativity. Accordingly, we shall examine the role of covariance principles in pre-relativity 
physics, in special Relativity and, only thereafter, in the Theory of General Relativity.

A prima facie indication of the factual content of covariance principles is that the commonly accepted interpretation of the Principle of special Relativity is to the effect that the covariance group of electrodynamics must contain the invariant velocity $\underline{c}$ which is interpreted as the velocity of electromagnetic propagation. But since the only invariant velocity of the Galilei group is an infinite velocity, it is obvious, on experimental grounds, that electromagnetic theory is not Galilei covariant. Thus, it would seem that there may be at least indirect experimental confutation of a covariance principle. of course, it is still logically possible to follow the line advocated by Bunge which would presumably consist in arguing that to say that Maxwell's theory is not Galilei covariant is obviously to say something about Maxwell's theory but to say nothing about the world. However, Maxwell's laws are not formulae that have been picked from a hat but purport to be reasonably accurate descriptions of something called the electromagnetic field. It might make a difference to say that a purportedly true description of reality is not Galllei covariant. Surely that is to say something, at least indirectly, about the world. In any event, the invariance of $c$ under velocity mappings is surely a brute fact, and no theory 
which incorporates that fundamental fact can admit the Galilei group of transformations.

Actually, it is not our intention to engage in polemics. On the contrary, the purpose of this dissertation is to argue for a middle ground between the two opposed points of view, each of which contains part of the truth. The general Iine which we intend to pursue will be clarified in a cursory way in the remainder of this chapter.

The Principle of General Covariance asserts that any genuine fundamental law of nature must hold regardless of the coordinatization which we happen to adopt to describe the physical system of interest. A physical theory should be as indifferent to the coordinate system as it is to the language of its author. Accordingly, a well-formed basic law should retain its form under an extremely wide class of coordinate transformations. For obvious reasons, such transformations must be differentiable and with a non-vanishing Jacobian. Apart from the latter requirements, they are quite arbitrary. It is easily shown that such a class of mathematically admissible coordinate transformations is the realization of a group. We may call it the general covariance group or, following Anderson, the manifold mapping group. The requirement that a theory be covariant under the manifold mapping group is the Principle of General Covariance, in a nutshell. 
It is a most fortunate aspect of the wedding of physics and mathematics that there is a class of geometrical objects, namely tensors, which form a basis for the realization of the manifold mapping group. Accordingly, the requirement that the basic laws (postulates) of a theory be expressible as tensor equations is equivalent to the requirement of general covariance. But the essential meaning of the general covariance of a law is that it be covariant under any mathematically admissible substitution, as defined above. Hence, it would appear to follow that Maxwell's laws, which are expressible as tensor equations, are covariant under the group of Galilei transformations - a result which is

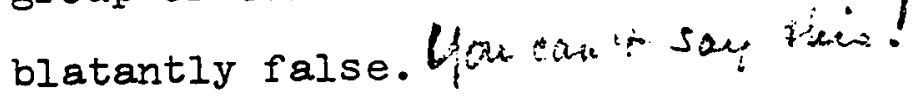

The germ of the solution to this paradox is actually hinted at in Einstein's original paper of 1916 in which he had the insight to draw a distinction between the Principle of General Covariance and the Principle of General Relativity. Since Kretschmann's paper, however, everyone seems either to have ignored the distinction completely or, at least, to have treated the Principle of General Covariance as the mathematical model of the physical Principle of General Relativity. However, even the latter is a serlous mistake. Bunge, in his Foundations of Physics, takes considerable pains to emphasize the distinction between a coordinate system,

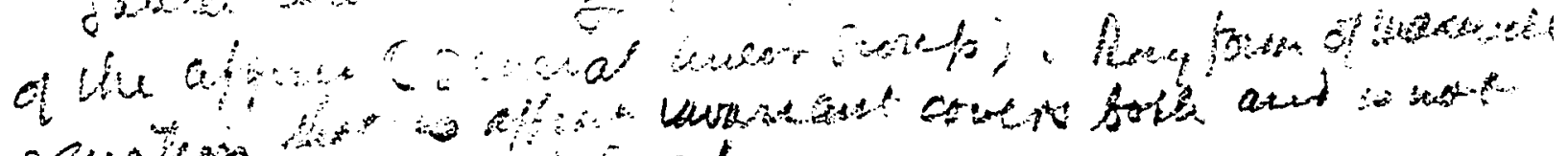

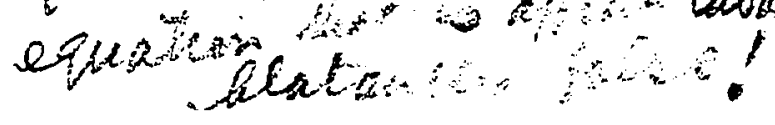


which is a conceptual object of mathematics, and a frame of reference, which is a physical object such as the walls of a laboratory. Roughly, the distinction between covariance and reliativity corresponds to that between coordinate systems and frames of reference. The Principle of General Relativity is a physical hypothesis which may be stated in the negative form that there is no privileged frame of reference to be found in nature or in the positive form that all frames of reference are dynamically equivalent. Since every frame of reference may be coordinatized, it follows that if a law satisfies the Principle of General Covariance it will, a fortiori, satisfy the Principle of General Relativity. However, the two principles are not to be confused. They are by no means coextensive.

The frequent references to general covariance in the literature of relativity physics is partly due to the availability of a mathematical formalism ideally suited to obtain generally covariant laws. However, this formalism - we naturally refer to tensor analysis - must be treated with great care or it will lead to a serious misunderstanding of relativity physics and the consequent controversies which this dissertation should serve to dispell. In any case, the physicist should not be concerned with general covariance for its own sake but only with those particular instances of covariance which have to do 
with possible frame transformations. These considerations are well exemplified by the case in hand of the generally covariant form of Maxwell's laws. The source of the mistake was to forget that the sole significance of the generally covariant formulation of a law for the physicist is to demonstrate that the law is independent of the frame of reference to which it happens to be referred. As we shall show, especially in Chapter VIII, the acceptance of a physically unrestricted general covariance for physical theories would permit the introduction of frames of reference which could have no counterpart in the physical world. It is precisely with reference to such frames, i.e. ones which contravene laws of physics, that Maxwell's equations could be Galilei cojariant.

our general conclusion will be that Bunge is formally correct in regarding covariance principles, per se, as devoid of factual content. In this context, they are nothing but metatheorems of pure mathematics. On the other hand, we shall argue that once we speak of the relationshtps which hold between frames of reference, whether inertial or arbitrary, we have moved from the realm of pure mathematics to that of physics. When we make assertions about the group properties of frame transformations, we are referring to concrete groups which are realized in the physical world. Such assertions certainly 
do have a factual content. This dissertation will consist in the elaboration of the fundamental topics which have been alluded to in this introductory chapter. 


\section{CHAPTER II}

TECHNICAI PRELIMINARIES

The purpose of this chapter is twofold. In the first place it should serve to introduce the reader whose training is primarily philosophical to some of the most basic notions of applied mathematics and theoretical physics which happen to play a fundamental role in the ensuing discussions. However, this chapter should not be ignored by the scientific reader, since some of the fundamental concepts are obscured by ambiguity. The second function of this chapter is, accordingly, to state unequivocally what the present writer intends to signify by certain conceptual terms which will be employed throughout the remainder of this dissertation. 1. Coordinate Systems and Coordinate Transformations:

A very wide class of physical theories concerns the behaviour of physical systems, such as bodies and fields and a combination of the two, in space and time. We may designate this the class of space-time theorles. A law of space-time physics will describe the changing spatial configuration of a physical system in the course of time. Accordingly, it is necessary to label the position or configuration of a system at any point in time. In elementary physics, the standard method of labeling is to utilize a Cartesian coordinate system. 
This conceptual entity consists of three mutually orthogonal, rectilinear axes. Each axis is labelled by a variable. Thus, the three variables $\underline{x}, \underline{y}, \underline{z}$ may represent the spatial axes of a particular system of coordinates. In somewhat more advanced treatments a single letter with a subscript or superscript will perform the same function, viz., $x^{i}(1=1,2,3)$. The $x^{i}$ are called the coordinate variables. More precisely, a coordinate is a function $x^{i}$ on the given space to some field, usually the real line, such that $x^{i}\left(b^{j}\right)=\delta_{i j}$ where the $b^{j}$ are the base vectors and $\delta_{i j}$ is the Kronecker symbol. Clearly, every point of a three-dimensional manifold will be uniquely labelled by an ordered triple of three numbers specifying a particular value of each of the coordinate variables. When a manifold is thus associated with a set of coordinates, it is said to be coordinatized.

It is obvious that no coordinatization of a given manifold is unique. Given a particular Cartesian coordinate system, it is possible to produce an infinitude of further systems by means of a change of origin of the original system or by a rotation about the origin or by a combination of these. In general, such a mathematical operation will send every ordered triple of numbers associated with a point of the manifold into a distinct ordered triple assoclated with the same point. Such a 
change in the coordinatization of the manifold is called a coordinate transformation. The set of functions which maps the first set of triples onto the second set is called the transformation equations. In general, each member of the second or 'barred' triple is a function of every member of the first triple. Consider two given coordinatizations, $S$ and $\bar{S}$, of the manifold and a mapping from $S$ onto $\bar{S}$. Let $x, y, z$ be the spatial coordinates of a point with respect to $S$, and $\bar{x}, \bar{y}, \bar{z}$ be the spatial coordinates of the same point with respect to $\bar{S}$. Then, given that the transformation is linear, the transformation equations will be of the general form:

$$
\begin{aligned}
& x \rightarrow \bar{x}=c_{11} x+c_{12} y+c_{13} z+\bar{x}_{0} \\
& y \rightarrow \bar{y}=c_{21} x+c_{22} y+c_{23} z+\bar{y}_{0} \\
& z \rightarrow \bar{z}=c_{31} x+c_{32} y+c_{33} z+\bar{z}_{0}
\end{aligned}
$$

The $c_{i k}$ are the direction cosines between the various axes of the two systems of reference, and $\overline{\mathrm{x}}_{0}, \overline{\mathrm{y}}_{0}, \overline{\mathrm{z}}_{0}$ are the coordinates of the origin of $s$ with respect to $\bar{s}$. The class of coordinate transformations so far considered is actually in reality: very restricted. In addition to the transition from one Cartesian system to another, we must take account of transitions between arbitrary curvilinear systems, in which case the $c_{1 \mathrm{k}}$ may be very much more complicated. For typographical convenience in the remainder of this section the coordinate variables of the unbarred system will be denoted by $x^{1}$ and 
those of the barred system by $y^{i}$. We may also make the trivial generalization to an n-dimensional manifold. Accordingly, we now consider the class of time-independent, real, single-valued, reversible transformations of the form:

$$
T: x^{i} \rightarrow y^{i}=y^{i}\left(x^{1}, x^{2}, \ldots, x^{n}\right) \quad(i=1,2, \ldots, n)
$$

Since, for reasons which will shortly emerge, we are concerned only with those transformations which are realizations of a group, the assumption of reversibility is a necessary one, in which case we write the inverse transformation:

$$
T: y^{i} \rightarrow x^{i}=x^{1}\left(y^{1}, y^{2}, \ldots, y^{n}\right)
$$

The conditions which must be imposed on the functions $y^{i}(x)$ are, firstly, that they be first-order differentiable in a finite region $\mathrm{R}$ of the $\mathrm{n}$-dimensional manifold $v_{n}$, which we shall denote by saying that the $y^{1}(x)$ are of class $C^{1}$ in the region $R$. Secondly, we require that the functional determinant (Jacobian), $J=d f .\left|\frac{\partial y^{i}}{\partial x^{i}}\right|$, does not vanish at any point in $R$. This will suffice to ensure not only the existence of a single-valued inverse but also that the $x^{i}(y)$ are of class $c^{1}$ in $R$. If the manifold has a Euclidean structure, these requirements would not be restricted to a finite region but could easily be made to apply to the entire $V_{n}$ by the simple expedient of employing cartesian coordinate systems. All transformations, whether linear or not, which satisfy the foregoing conditions constitute the class of mathematically 
admissible transformations. However, the reader should be warned that the designation 'mathematically admissible' carries no physical warrant. We shall find in subsequent chapters that it is necessary to impose additional restrictions to define the class of physically admissible transformations which is, therefore, a subset of the set of transformations here defined.

We shall conclude this section with a fairly crude but adequate sketch of the proof that the set of mathematically admissible transformations form a group. It should be emphasized that this is not a trivial

conclusion, since there are distinguished subsets of the set of mathematically admissible transformations which lack the group property.

Lemma: If $\mathrm{J}$ and $\underline{K}$ are the Jacobians of $\underline{T}$ and $\underline{T}^{-1}$ respectively, then $\frac{\text { JK }}{1}=1$.

By definition of $T$ and $T^{-1}$, there follow the identities: $y^{i} \equiv y^{i}\left[x^{1}\left(y^{1}, y^{2}, \ldots, y^{n}\right), \ldots, x^{n}\left(y^{1}, y^{2}, \ldots, y^{n}\right)\right]$

Differentiating with respect to $\mathrm{y}^{j}$ :

$$
\frac{\partial y^{i}}{\partial y^{j}}=\frac{\partial y^{i}}{\partial x^{n}} \frac{\partial x^{n}}{\partial y^{j}}=\delta_{j}^{i} \text {, where } \delta_{j}^{i} \text { is the Kronecker symbol. }
$$
But

$$
\left|\frac{\partial y^{i}}{\partial x^{k}} \frac{\partial x^{k}}{\partial y^{k}}\right|=\left|\frac{\partial y^{i}}{\partial x^{k}}\right| \cdot\left|\frac{\partial x^{k}}{\partial y^{j}}\right|=\left|\frac{\partial y^{i}}{\partial x^{k}}\right| \cdot\left|\frac{\partial x^{i}}{\partial y^{k}}\right|=J K=\left|\delta_{j}^{i}\right|=1
$$

Lemma: If $T_{1}$ and $T_{2}$ are admissible transformations, the product ${ }_{2}{ }_{1}{ }_{1}$ is also an admissible transformation.

Consider two transformations:

$$
\begin{aligned}
& T_{1}: x^{1} \rightarrow y^{1}=y^{1}\left(x^{1}, x^{2}, \ldots, x^{n}\right) \\
& T_{2}: y^{1} \rightarrow z^{1}=z^{1}\left(y^{1}, y^{2}, \ldots, y^{n}\right)
\end{aligned}
$$




$$
\begin{aligned}
& T_{3}=T_{2} T_{1}: x^{i} \rightarrow z^{1}=z^{i}\left[y^{1}\left(x^{1}, x^{2}, \ldots, x^{n}\right), \ldots, y^{n}\left(x^{1}, x^{2}, \ldots, x^{n}\right)\right] \\
& J_{3}=\left|\frac{\partial z^{i}}{\partial y^{k}} \frac{\partial y^{k}}{\partial x^{j}}\right|=\left|\frac{\partial z^{i}}{\partial y^{k}}\right| \cdot\left|\frac{\partial y^{k}}{\partial x^{j}}\right|=\left|\frac{\partial z^{i}}{\partial y^{j}}\right| \cdot\left|\frac{\partial y^{i}}{\partial x^{j}}\right|=J_{2} J_{1}
\end{aligned}
$$

Theorem: The set of mathematically admissible transformations form a group.

(i) The product of two admissible transformations is an admissible transformation.

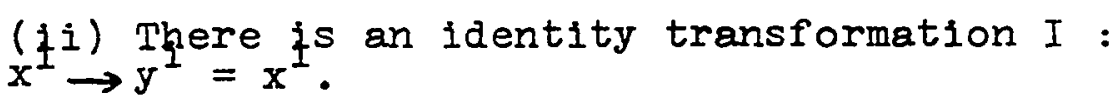

(iii) For any transformation there is an inverse such that $T^{-1} T=I$

$$
\text { (iv) } T_{3}\left(T_{2} T_{1}\right)=\left(T_{3} T_{2}\right) T_{1}
$$

2. Frames of Reference:

The distinction between frames of reference and coordinate systems tends too often to be either overlooked or forgotten by physicists. The blurring of this fundamental distinction can lead to errors of both fact and philosophy. In particular, we shall find that the significance of the various principles of relativity is apt to be misunderstood when this occurs.

A coordinate system is a purely conceptual object of mathematics which, like all such objects, is devold of intrinsic physical significance. It may, however, be assigned a physical meaning when it is employed as the conceptual model of a physical frame of reference.(Bunge, 1967a, pp. 103-105; Møller, 1952, pp. 234ff.) A frame of reference is a physical entity of some kind such as the earth, the sun, a laboratory or even the centre of mass of a system of material objects. (strictly speaking, the 
latter is just a reference point.)

Physical space has no intrinsic cartographic properties. Consequently, one cannot specify the absolute position of an object, i.e. its position vis à vis space. To specify a position, it is necessary to secure a point of reference which is arbitrarily treated as fixed. This fixed point may then be utilized as the origin of a physical frame. It is at this juncture that a coordinate system is usefully introduced as the mathematical model of the frame of reference. For example, if one decides to adopt a heliocentric frame of reference, one may then specify the position of an object with respect to the sun, but not literaliy with respect to the system of coordinates.

It follows from the foregoing remarks that the coordinatization of a reference frame is not unique. In principle, infinitely many systems of coordinates may be associated with a single frame of reference(Bunge, 1967a, p. 104). By the same token, the set of frame transformations is not coextensive with the set of coordinate transformations. It is frequently necessary in the practice of physics to refer a physical system to a new frame of reference. For example, it may be desired to effect a transition from a laboratory frame to a centre of mass frame, $0 i$ from a geocentric to a heliocentric frame. The mathematical procedure is, in principle, quite straightforward. A coordinate system which models the original 
frame of reference is first specified. Then the second reference frame is likewise modelled by a suitable system of coordinates. Finally, the set of transformation equations between the two sets of coordinates is determined. These transformation equations must model the relationship between the two frames of reference. Consequently, it would be correct to speak of them as transformation laws. The set of coordinate transformations which model frame transformations is a minute subset of the set of mathematically admissible coordinate transformations. Many types of coordinate transformations which are employed to achieve the optimum of mathematical tractability have nothing to do with frame transformations. We may, for example, transform the description of an electrostatic system from rectangular to spherical coordinates to facilitate the solving of Poisson's equation. In particular, transformations of this kind are purely mathematical and should not be regarded as laws in the physical sense. On the other hand, when we deal with the relationship between two distinct frames of reference, we are involved with the behaviour of two physical systems, In which case It is proper to speak of the laws which relate them. The mark of a physically significant frame transformation, as distinct from one which is a mere analytical aid, is the occurrence of the time parameter in the equations of transformation. In the equations(2.1), 
it was tacitly assumed that the coefficients $c_{i k}$ were constant. However, if these equations modelled the relationship between two frames of reference in relative motion, the $c_{i k}$ would obviously vary in time. Moreover, their explicit form would depend on the type of motion involved, e.8. rotational or translational, accelerated or uniform.

3. Scalars, Vectors and Tensors:

(i)scalars: A scalar is a one component geometrical object. Once a number has been assigned to a scalar magnitude on the basis of a particular scale, its value is fixed independently of the coordinate system. This notion may be immediately extended to that of a scalar field. The latter may be represented in explicit numerical form as, for example, in the case of a classical temperature distribution throughout a given region or, analytically, as a function of the coordinate variables. Probably, the most fundamental example of a scalar in space-time physics is the square of the line element, known as the interval: $(d s)^{2}=g_{1 k} d x^{1} d x^{k}$. It should not be supposed, however, that a scalar is an object without a transformation law. All geometrical objects are defined by the manner of their transformation. Moreover, not all one component objects are scalars. For example, the transformation law of $a$ pseudo-scalar differs from that of a scalar. Moreover, the individual components of vectors:and tiensors are not 
geometrical objects at all. Consequently, they lack invariant significance. Accordingly, it is desirable to express the transformation law of a scalar formally, although it may seem trivial to the novice:

$$
\mathrm{x} \rightarrow \overline{\mathrm{x}}=\mathrm{x}
$$

(ii) Contravariant Vectors: Take two infinitely close points whose coordinates are $x^{i}$ and $x^{i}+d x^{i}$ respectively, with respect to a given coordinate system $S$. The infinitesimal displacement between the two points in $S$ is given by $\mathrm{dx}^{i}$. Let us transform from $s$ to $\bar{s}$ by a mathematically admissible transformation:

$$
x^{i} \rightarrow \bar{x}^{i}=\bar{x}^{i}\left(x^{1}, x^{2}, \ldots, x^{n}\right)
$$

By differentiation of (2.2), we obtain:

$$
d \bar{x}^{i}=\frac{\partial \bar{x}^{i}}{\partial x^{j}} d x^{j}
$$

Equations (2.3) are the transformation law for coordinate differentials. In general, any object $A^{1}$ whose law of transformation is:

$$
\bar{A}^{j}=\frac{\partial \bar{x}^{j}}{\partial x^{i}} A^{i}
$$

will be called a contravariant vestor. It follows immediately from the linear and homogeneous character of the transformation (2.4) that if $A^{i}$ and $B^{1}$ are two contravariant vectors, then $\mathrm{pA}^{1}+\mathrm{qB}^{1}$ is also a contravariant vector, where $p$ and $q$ are arbitrary constants. (iii) Covariant Vectors: From a given scalar field $\phi\left(x^{i}\right)$, we may form the $n$ quantities $A_{i}$ by differentiation:

$$
A_{i}=\frac{\partial \phi}{\partial x^{i}}
$$


which is nothing but the n-dimensional generalization of the familiar operation of forming the gradient of a scalar field in three dimensions. By the product rule of differentiation, we may determine the transformation law of the $A_{1}$ :

$$
\bar{A}_{j}=\frac{\partial \rho}{\partial \bar{x}^{j}}=\frac{\partial x^{i}}{\partial \bar{x}^{j}} \frac{\partial \rho}{\partial x^{i}}=\frac{\partial x^{i}}{\partial \bar{x}^{j}} A_{i}
$$

In seneral, any quantity with the transformation law (2.5) is called a covariant vector. (iv) Tensors: Let us consider a product of arbitrary covariant and contravariant vectors, e.g. $A^{i} B^{j} C_{K}$. It is easily shown that the transformation law for this quantity from $S$ to $\bar{S}$ is a simple generalization of $(2.4)$ and (2.5).

$$
\bar{A}^{p} \bar{B}^{q} \bar{C}_{r}=\frac{\partial \bar{x}^{p}}{\partial x^{i}} \frac{\partial \bar{x}^{q}}{\partial x^{j}} \frac{\partial x^{k}}{\partial \bar{x}^{r}} A^{i} B^{j} C_{k}
$$

The quantity $A^{1} B^{j} C_{k}$ is a geometrical object which may be signified by $\mathrm{T}_{\mathrm{k}}^{i j}$. Thus, we may rewrite the transformation Law $(2.6)$ as :

$$
\bar{T}_{r}^{p q}=\frac{\partial \bar{x}^{p}}{\partial x^{i}} \frac{\partial \bar{x}^{q}}{\partial x^{j}} \frac{\partial x^{k}}{\partial \bar{x}^{r}} T_{k}^{i j}
$$

Any quantity with the transformation law (2.7) is said to be a tensor of the third rank, twice contravariant and once covariant. The generalization to tensors of any rank and type is obvious. A vector is a tensor of rank one and a scalar is a tensor of rank zero.

It is especially noteworthy that the transformation law of a tensor is linear and homogeneous. Accordingly, the algebra of tensors will have the usual properties associated with objects of that kind. The 
reader whose knowledge of mathematics is fairly elementary should note, in particular, that the linear character of the transformation coefficients holds even though the transformation of interest may, itself, be non-linear.

From the homogeneous character of tensors, it follows that if a tensorial quantity is zero in one system of coordinates, it will remain zero in every system of coordinates. The self-evident consequence of this is that a tensor equation which is valid in one system of coordinates must be valid in every system which is obtainable therefrom by a mathematically admissible transformation. Accordingly, a common stratagem of mathematical physics is to formulate a law as a tensor equation with respect to a system of coordinates in which it takes the simplest possible form. It then follows from the tensorial character of the equation that it will be valid for all admissible systems of coordinates. However, it is important to note that, in general, this mathematically privileged coordinate system only holds locally so that it will not normally represent a physical frame of reference. We shall appreciate the significance of the last remark in Chapter VIII. The great utility of the mathematical theory of tensors lies in its providing a means of writing laws of nature in a way that is independent of any particular system of coordinates. This, 
of course, is an extension of the elementary theory of Cartesian vectors on the basis of which it is possible to express laws of nature which are independent of any orthogonal system of coordinates. 4. Fundamental Laws:

The notion of a fundamental law is crucial to the elucidation of such concepts as those of covariance and inertial frame of reference. A commonly accepted definition of a fundamental law is one that contains no individual constants, i.e. names of particular individuals, in its formulation. While we accept this definition, we find it desirable to express it in terms which pertain directly to physical theory. The motivation of the definition is that a basic or fundamental law is of global significance, holding for all times and all regions. Thus, the occurrence of the name of a particular time or region or physical objecti in a statement precludes its having a fundamental character. It follows that the solutions of fundamental laws cannot, themselves, be fundamental sirce they depend essentialiy on initial and/or boundary conditions. Similarly, a law which singles out a particular frame of reference as essentially privileged would not be fundamental since it would contain, at least by implication, the name of the reference frame. On this bas1s, any law whlch refers essentially to a particular material medium wolild be of a non-fundamental kind. In an 
axiomatic development of a physical theory, laws in which names occur essentially would have the status of low level theorems. Thus, an axiomatic development of a theory is by no means arbitrary as to the choice of axioms. For example, the law of sound propagation depends essentially on the characteristics of the medium of propagation, e.8. the air, which serves as a privileged frame of reference, automatically precluding it from the class of fundamental laws.

By the same token, a law statement which contained terms which depended essentially on the state of motion of a particular frame of reference would be non-fundamental since it would presumably hold not globally but only in certain regions and at certain times which would have to be specified. 5. Invariance and Covariance:

A well-known property of groups in general is the existence of certain invariants which may serve to define a given group. Let $s$ be a set of objects which are operated on by the elements of a group $G$. Iet $x^{\prime}$ be the image of $x \in S$ under an arbitrary mapping of $G$. Let $F$ be a given function of $x$. Then the function $F$ is an invarlant of $G$ if and only if $F(x)=F\left(x^{\prime}\right)$. For example, $F(x, y, z)=x^{2}+y^{2}+z^{2}$ is an invariant of the group of spatial rotations, 1.e. the three-parameter orthogonal group. Alternatively, if $x$ is any object and $g(x)$ is an 
operation on $x$ by an arbitrary element of $G$, then $x$ is an invariant of $G$ if and only if $x-g(x)=0$.

The invariants which are associated with the various groups of transformations are of distinct physical and epistemolopol interest. In the first place, it is intuitively clear that any quantity that refers to an invariant property of the world should be independent of the choice of coordinates. A coordinate system is, figuratively speaking, a language employed for the space-time description of a physical system. Just as nature is indifferent to the choice of natural language which happens to be employed to describe it, so it should also be indifferent to the physicist's cholce of technical language, e.g. coordinate system. Now if a property of an object is independent of the coordinatization with respect to which it is expressed, it is, a fortiori, invariant under a group of coordinate transformations. We often refer to such invariant properties as the symmetries of the object. We conclude that the discovery of the symmetries or invariant properties of a physical system, e.8. the total electric charge, has: a fundamental significance in the theoretical descriptions of objective reality.

Closely allied to the concept of invariance, but on no account to be confused with 1t, is that of the covariance of lawlike statements. A physical law may be 
regarded as an hypothesis which purports to describe relationships or patterns which hold objectively between given physical magnitudes. Such magnitudes are not, in general, invariant. That is to say, they will have distinct numbers assigned to them by distinct coordinatizations. Nevertheless, the pattern of the relationship is still objective. For example, an interparticle distance may be expressed in the form: $s^{i j}=\left|x^{i}-x^{j}\right|$, where the $x^{i}, x^{j}$ are the cartesian spatial components of the two particles. Under a coordinate rotation, the numbers assigned to the $x^{1}, x^{j}$ will change but the quantity $s^{i j}$ will be invariant.

Bunge has drawn the useful distinction between laws $_{1}$ and laws $_{2}(e .8 .1963$, ch. 10). Laws 1 are the objective patterns of physical reality, while laws, are the scientific hypotheses which purport to represent them. Just as the magnitude of an invariant is independent of the coordinatization, so a law must be similarly independent. But if $a$ law $_{1}$ is independent of the cholce of the coordinate system, such independence must be mirrored by the corresponding ${ } \mathrm{aw}_{2}$.

Specifically, while the various terms which compose the law2 may change under a given group of transformations, the functional relationships holding between these terms should remain fixed as a reflection of the objectivity of the law $_{1}$. An added complication in 
the case of the covariance of laws is that the functions which occur in them, $\epsilon . g$. field variables, will also in general be transformed. Let us suppose that we are given a law of the form:

$$
F\left(\varphi, \psi, \ldots, \frac{\partial \varphi}{\partial x^{i}}, \frac{\partial \psi}{\partial x^{k}}, \ldots\right)=0
$$

Under a group of transformations $G$, not only will the $x^{i}$ undergo change but also the $\varphi, \psi, \ldots$, which may, as we said, be field variables. Now (2.8) is said to be covariant if and only if:

$$
F\left(\bar{\phi}, \bar{\psi}, \ldots, \frac{\partial \bar{\phi}}{\partial \bar{x}^{i}}, \frac{\partial \bar{\psi}}{\partial \bar{x}_{k}}, \ldots\right)=0
$$

That is to say, the transformed functions of the transformed arguments are isomorphic with the original functions of the original arguments. We shall subsequently have reason to question this account but it happens to be the standard one, and the reader will gain a better appreciation of the concept of covariance when he discovers what is wrong with it.

It is unfortunate that the subject of covariance is neither as straightforward nor as uniformly comprehended by physicists and philosophers as one might wish. At this point, we shall merely touch on some of the more obvious difficulties. Firstly, there is a fundamental ambivalence in the use of the word 'covariance', which appears to stem from what happens to be regarded as the basis for the requirement of covariance on physical laws. The philosopher and mathematician tend to 
view covariance as a sweeping epistemological desideratum to the effect that a genuine law of nature must be absolutely independent of its mode of description. Hence, any coordinate system, no matter how exotic, should be capable of serving as an adequate vehicle for a covariant space-time description of the world. The physicist, on the other hand, views the covariance of physical laws as both practically and theo retically desirable in order that they may be expressed in a form which is independent of the frame of reference. In short, while the philosophicomathematical interest in covariance tends to be focussed on coordinate transformations, that of the physicist, inadvertently or otherwise, has tended to be more concerned with frame transformations.

It is true that the general mathematical requirement may be achleved by writing laws in the tensor notation. But that highly abstract and compressed formalism may obscure more than it reveals if the physical bas is of the theory of interest is overlooked. A tensor equation may be factualiy false even though it is mathematically well-formed. In any event, covariance would have been a viable requirement even in the absence of the tensor calculus; and it is a fact that when more elementary mathematical tools are employed, the fundamental laws of physics fail to satisfy the requirement of general covariance. As we have already noted, coordinate 
transformations, per se, are normally employed from the relatively trivial motive of computational facility. While this matter deserves more discussion, we shall, for reasons which will emerge later, utilize the term 'covariance' to designate the formal requirement that the fundamental laws be independent of their coordinatization. The more specialized requirement that fundamental laws be independent of the frame of reference to which they are referred, which has nothing directly to do with the 'langlage' in which they are expressed, we shall call the principle of relativity, which is discussed in section? of this chapter. It is obvious that while frame transformations are complicated by their time-dependent nature, they need not involve a transition to a different kind of coordinate system, e.g. Cartesian to spherical. However, even when we agree to restrict the. requirement of covariance to the area of physically significant frame transformations, elements of ambiguity persist. Suppose that we take the requirement to mean simply that the form of a law be the same in every frame of reference. In Classical Mechanics as well as in Special Relativity Mechanics, covariance is supposed to pertain only to inertial frames of reference. Thus, if a fundamental law holds in a given frame of reference, it may not hold in a frame which is rotating with respect to the original frame. However, even in such cases, it will

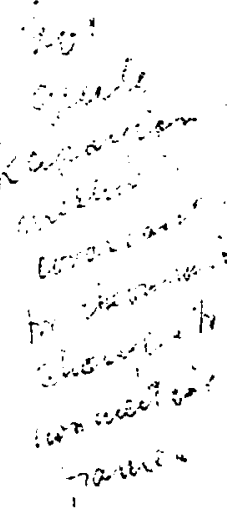


always be possible to preserve the form invariance of the law of interest in a formal or vacuous fashion. For example, if we consider a laboratory frame $K$ and a centre of mass frame $\bar{K}$, the expression for the total angular momentum of a system of particles is given in $\mathrm{K}$ by:

$$
\vec{L}=\vec{R} \times M \vec{v}+\sum_{i} \vec{r}_{i} \times \vec{P}_{i}
$$

where the first term on the right is the angular momentum of the centre of mass and the ith. term in the summation is the angular momentum of the ith. particle about the centre of mass. In terms of $\overline{\mathrm{K}}$, the first term will vanish, and the angular momentum will be given by:

$$
\overrightarrow{\bar{L}}=\sum_{i} \overrightarrow{\vec{r}}_{i} \times \overrightarrow{\bar{P}}_{i}
$$

However, if we so choose, we may retain the first term and claim that (2.10) is covariant under this particular transformation. Obviously, this would not have any effect on the results of our computations, since the first term would be identicaliy zero in the centre of mass frame. But there is nothing to prevent our adding any number of such terms to the expression of a physical law, so long as we are concerned only with the results of computations. Consider, for example, the general case of the equation of motion of a point-particle with respect to a frame which has both linear and angular acceleration with respect to an inertial frame. Let the position vector of the particle be $\vec{R}$ in the inertial frame and $\vec{r}$ in the non-inertial frame. Accelerations and velocities with 
respect to the non-inertial frame are enclosed in square brackets. A tedious but elementary calculation yields the following general equation of motion:

$$
m[\ddot{\vec{r}}]=\vec{F}-m \vec{A}_{0}-2 m \vec{\omega} \times[\dot{\vec{r}}]-m[\dot{\vec{\omega}}] \times \vec{r}-m \vec{\omega} \times(\vec{\omega} \times \vec{r})
$$

where $\vec{A}_{0}$ is the acceleration of the origin of the moving frame with respect to the inertial frame, and $\vec{F}=m \ddot{\vec{R}}$. The second term on the right hand side is due to the Iinear acceleration, while the succeeding terms are respectively the Coriolis, the transverse, and the centrifugal terms. Now, (2.12) is the law of motion of a particle in Newtonian Mlechanics, expressed in vectorial form, which is covariant under arbitrary frame transformations. In the more specialized cases, the various terms simply become zero.

Now the procedure that we have followed might be ruled out by the simple declaration that every term in a meaningful physical law must be non-vanishing under a coordinate transformation. But such a stratagem would be without any foundation. For example, continuity equations may be written in a form in which they assert that a certain quantity vanishes identically. A more tenable position would be to hold that (2.12) is not a fundamental law. The reader will recall that we stipulated that a fundamental law must not contain individual names, even covertly. But, clearly, every term on the right hand side of (2.12), with the exception of the first, refers 
specifically to the particular non-inertial frame. For this reason, we are obliged to discount (2.12) as an example of a covariant law of nature, since it describes the state of affairs in a particular frame of reference rather than a pattern of global significance. Accordingly, we shall keep in mind throughout this dissertation that all references to covariant and relativistic laws are to be understood as applicable to fundamental laws only(Cf. Bunge, 196?as pp. 86f.). As we have amply illustrated, in the absence of such a restriction, the entire discussion would be trivialized. 6. Inertial Frames of Reference:

In all space-time physical theories, with the possible exception of General Relativity, a certain class of reference frames plays a fundamental role. We refer, of course, to inertial frames of reference. The concept of inertial frame is familiar to every student of physics. It exemplifies those concepts which are intuitively clear yet often difficult to define with adequacy. Bergmann describes them as "frames in which the laws of nature are fundamentally simple, that is, in which the laws of nature contain fewer elements than they would otherwise."(1942, p. xiv) According to Møller, an inertial frame is one in which Newton's first law is valid(1952, p. 1). Fock seems to say the same thing in holding that it is a frame "in which the motion of a body 
is uniform and rectilinear, provided no forces act on 1t."(1964, p. 15) Anderson of ers a more sophisticated version of this, in defining an inertial frame as one in which the components of the affinity in the equation of motion of a free particle vanish(1967, p. 113). Bunge

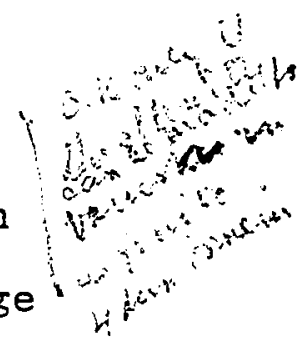
takes a rather different tack in defining an inertial frame with respect to a given theory as one that satisfies all of the axioms of that theory(1967a, p. 136). Thus, he would make a systematic distinction between a mechanical inertial frame, an electromagnetic inertial frame, and a gravitational inertial frame.

In the first place, we are bound to agree with Bunge, who appears to maintain tacitly that it is unsatisfactory to define an inertial frame in terms of a single law. It is clearly more realistic to define it in terms of the complete set of basic laws of a theory. In this way, for example, even the geocentric frame is not deprived of its local, quasi-inertial character by the presence of a gravitational field. Strictly, on the other definitions, an inertial frame would be infinitely distant from all matter and would, itself, be non-material in short, a pure abstraction: Nevertheless, Bunge's treatment of the concept of inertial frame is lacking in one important respect. Bunge treats of mechanical, electromagnetic and gravitational inertial frames but falls to deal with the general 
concept of inertial frame in which, clearly, neither a single law nor even a complete set of basic laws may occur essentially. To form an adequate appreciation of the role of inertial frames in various theories, it is first necessary to have a clear idea of the general concept, itself. In fact, this presents no great difficulty. Intuitively, an inertial frame is one which has no influence on the physical processes which occur in it or with respect to it. In more precise language, it is any frame of reference in respect of which the fundamental laws of a given theory contain no terms which depend essentially on 1t. To take the simplest of examples, by way of. illustration, Newton's second law has the well-known form, $\vec{F}=m \vec{a}$, in any inertial frame. If we refer the same mechanical process to a frame which has the linear acceleration $\vec{a}_{0}$ with respect to the first frame, the description of that process must be reformulated as:

$$
\vec{F}=m \vec{a}-m \vec{a}_{0}
$$

Clearly, the second term on the right hand side of (2.13) depends essentially on the motion of that particular frame of reference, whence that frame is non-inertial. In the second place, the reader should be reminded that, consonant with what was said about frames of reference in general, the concept of inertial frame is not a mathematical notion but a physical one. Given a frame of reference in which the basic laws of a theory 
have been corroborated, any other frame which is in motion with respect to the original one and with respect to which the basic laws continue to hold is to be regarded as a member of the equivalence class of inertial frames of that thecry. That each member of such an equivalence class, with the exception of the inertial frames of General Relativity, is unaccelerating with respect to every other member is to be regarded as a fact of nature and not as a mathematical necessity. Furthermore, in pre-reiativistic physics, it was assumed that the physical coordinates of an inertial frame were related to those of any other such frame by a Galliean transformation. The nature of this transformation made such an assumption appear to be purely arithmetic. We shall contend, however, that its physical character was revealed by the mere fact of its being rejected in the light of physical theory. In other words, by the same token, we shall argue for the physical character of the Lorentz frame transformations. 7. Principles of Relativity:

It is a curiosity, if not a disgrace, that so much confusion should reign in popular philosophy over the meaning of the concept of relativity in physics. We have in mind, for the present, not the particular principles of relativity, e.g. Classical, Special, etc., but the connotation of the general concept of relativity. In fact, it is possible to distinguish diametrically 
opposite accounts of the meaning of relativity - those which occur in several works of philosophy as against those which occur, albeit rather sparsely, in works of physical theory. It is no futile task merely to place the two accounts side by side in order to clarify the situation somewhat.

A popular philosophical conception of relativity principles is that they are assertions to the effect that the statement of a physical law or the assignment of a number to a physical magnitude is meaningless unless the context of that law or magnitude is simultaneously specified. Thus, it would be pointed out that Newton's first law is devold of content unless a frame of reference be specified, since it refers to rectilinear motion, a concept which has no significance in the absence of a frame of reference. For example, we may take the edge of a room as a reference line and propose that any body which moves in such a way that the perpendicular distance between it and the edge of the room is constant throughout the motion is undergoing a rectilinear translation. However, if the room is, itself, rotating with respect to some other frame of reference, say the sun, then the motion of the object will be curvilinear with respect to the second frame. Hence, it is meaningless to assert that an object is in a state of rectilinear translation, tout court. One must specify the frame relative to which the 
motion is referred. This would be taken as an instance of the relativity of motion. Similar examples abound anent relativity of position, velocity, weight, etc. Thus, in summary, the philosophico-popular conception of a principle of relativity is that it asserts that some specifled concept is one whose 'meaning' may be given only relative to a frame of reference. In embarrassingly many cases, this point of view is speciously extended to a form of subjective idealism (Eddingtonianism) in which 'frame of reference' is replaced by 'observer'. Then it is declared that the physical world which we perceive does not exist objectively but only in the minc of the beholder, or that there are as many worlds as there are observers. However, it is not our purpose to criticize this misguided brand of idealism but merely to contrast the conception of relativity principles on which it is puportedly based with that connotation of relativity principles which is normally accepted by the physicist.

Whereas the amateur philosopher associates 'relativity' with the changes that properties of a physical system undergo when referred to different frames of reference, the physicist, interestingly, stresses the very opposite, namely that a pattern of phenomena (not necessarily the phenomena themselves) is duplicated in different frames of reference. The larger the class of phenomena whose laws are unchanged when referred to 
different frames, the broader the corresponding principle of relativity. Thus, the Principle of Special Relativity of Einstein is broader than the Principle of Classical Relativity of Newton, since it encompasses both mechanical and eleotrodynamical laws. Similarly, the wider the class of frames to which a principle of relativity refers, the deeper the principle is taken to be. Thus, the Principle of General Relativity is regarded as having deeper significance than elther of the restricted principles since it pertains to all frames of reference as distinct from the very restricted class of inertial frames. In fine, to the physicist, a principle of relativity is the expression of a common objective reality, independent of its context, to a greater or lesser degree, and certainly independent of any minds that happen to be in the vicinity. Apart from the discrepancy which exists between the common philosophical conception of the principles of relativity and that of the physicists, a more significant confusion exists in physics itself between a principle of relativity and a principle of covariance. A covariance principle asserts that the form of an equation remains the same under a specified group of transformations. A relativity principle asserts that the content of a physical law is unchanged with respect to a specific class of reference frames. What is the relation between the two? Too often, it is made to appear that if they are not 
identical, then the covariance principle entails the corresponding relativity principle. But this cannot be the case since, as we have already remarked, covariance is a formal condition, while a relativity principle has factual content.

The justification for the acceptance of the aforementioned entailment is that if a law $I$ is covariant under a group of transformations $G$, it must retain its form under the subgroup of $G$ corresponding to possible frame transformations. But while $L$, as stated, may have the covariance property $G$ and, therefore, suggest the existence of a corresponding relativity principle, it may actually turn out that the elements which compose the formal statement of $L$ have been endowed with the incorrect geometrical properties. Thus, a quantity which has been treated as a scalar in pre-relativistic physics may turn out to be a component of a four-vector in relativity physics. Such mistaken ascription of geometrical properties is certainly possible. Hence, we are not licensed to claim that given a law with certain covariance properties, it must conform, a priori, to a certain relativity principle. The latter is physical and testable, while the former is formal and, at most, a consistency requirement. A relativity principle could only be entailed by a covariance principle if, per impossibile, the law of interest were absolutely incorrigible. We shall now 
attempt a general definition of a principle of relativity. However, we stress that we are now defining only the general concept. The formal statements of the various particular principles of relativity will be substantially different from the following.

If (1) $I_{1}, \ldots, I_{n}$ are the basic laws of a theory $T$.

(11) A set of permissible frame transformations is the realization of a group $G$.

Then: $T$ is G-relativistic if and only if the content of $L_{1}, \ldots . .-\frac{1}{I_{n}}$ is unaltered by any transformation which is an element of $G$.

By the 'content' of a law, we intend to signify the objective state of affairs which is conveyed by its statement. In the present context, that of basic laws, such states of affairs are global patterns. In the case of low level laws, they would be frame-dependent states of affairs, and in the case of the substitution instances of the latter, they would be particular facts.

It should be noted that neither the concept of inertial frame nor that of covariance occurs in this definition of a principle of relativity. The concept of inertial frame is excluded for the following reasons. Firstiy, if the concept were understood in the commonly accepted sense, then the definition would be too restrictive, since $G$ would be limited to a group of linear transformations, which pertain only to restricted principles of relativity. Secondly, if the concept were understood in 
the more general sense, which we advocated in the previous section, in which linearity is taken to be a factual. rather than a mathematical aspect, then particularization to inertial frames would be redundant. On the other hand, It would have been possible to include the concept of covariance in our definition. However, this would have entailed a much lengthier and more cumbersome statement than is suitable for a definition, since covariance, as we understand it in this dissertation, tends to be tied to a particular mathematical formalism; and as we have seen, the mathematics is apt to be confused with the physics. 
CHAPTER III

LAWS AND METALAWS

As we noted in the introductory chapter, the fundamental issue of this dissertation hinges on the distinction between laws and metalaws. Actually, the distinction between object statements and metastatements has long been common coin in mathematical logic and philosophy. In the technical vocabulary of logic, the distinction between metastatement and object statement is a relative one. We express a statement $S$ in a language L. When we wish to make a statement about $S$ or about I as a whole, we must resort to a second language $I_{m}$ which is the metalanguage with respect to which $L$ is the object language. But, in turn, a statement about $I_{m}$ would belong to a different metalanguage, with respect to which $I_{m}$ would be the object language. In what follows, we depart somewhat from this usage in treating the two levels of discourse as absolute. That is to say, the language of science which refers to the world of objective facts will be called the object language not only in the technical sense but also in respect of the function which it performs. Thus, the metalanguage will signify the language we employ to talk about the scientific language, or that part of the latter in which Bunge's laws 2 are expressed. 
This distinction has been exploited by Bunge in its application to the subject of laws and metalaws. In fact, Bunge distinguishes several kinds of metalaw statements, and 1t would be advisable to spend a little time in exploring some of these refinements, since an adequate explication of the nature and role of metalaws in science is the most crucial philosophical prolegomenon to a satisfactory resolution of the problem before us. Since Bunge has been tilling virtually virgin soil, we shall begin with an exposition of his views which adheres very closely to his own writings(1959, Ch. 4; 1961; 1963, Ch. 12; 19670, V. I, Ch. 6, sec. 7).

The class of metalaw statements is obviously of extremely wide extension. It comprises any statement whose referent is a law or set of laws. Two of Bunge's examples serve to exhibit their diversity: "Maxwell's equations are invariant under space inversion."(1961, p. 519) and "Electricians employ Kirchhoff's laws."(Ibid.) However, the subclass of metalaws which is of particular concern to philosophers and physicists consists of lawlike metalaw statements, 1.e. laws of scientific laws, for which Bunge has coined the expression metanomological statement. It is Bunge's contention that since metanomological statements have as their referents laws rather than objective patterns of nature or, in his own Idiom, laws 2 rather than laws, they are not to be regardid 
as statements with factual content in the obvious sense. They refer not to the world but to our modes of representing the world.

Bunge distinguishes three principal kinds of metanomological statements, which he calls factual, normative and methodological. Additionally, factual metanomological statements are grouped into two varieties according to whether their truth value is mathematically demonstrable - the analytic variety, or decidable, a posterior1, on the basis of the actual features of the laws of the theory to which they are applied - the synthetic variety. The following diagram schematizes Bunge's classification of metalaw statements.

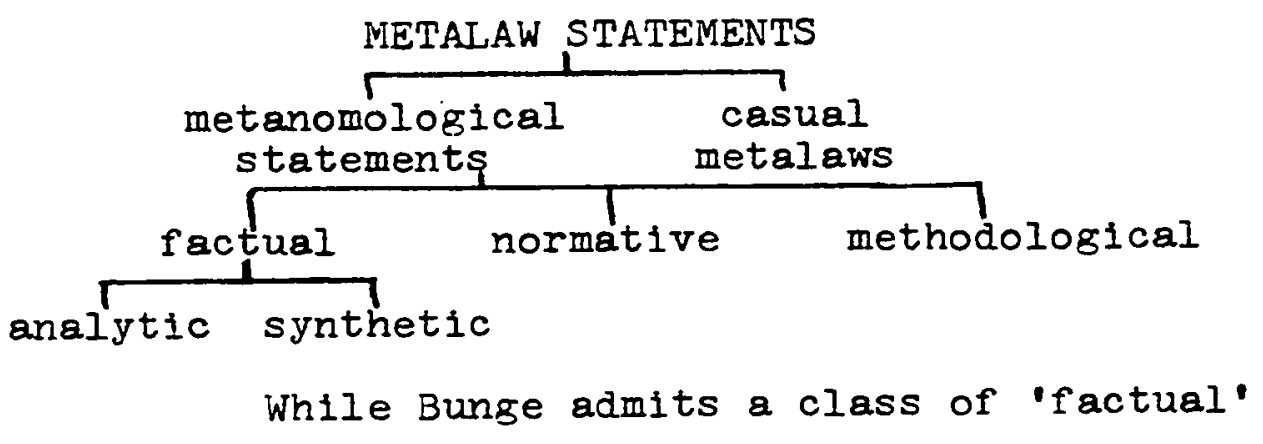
metanomological statements into his scheme, he cautions us not to be misled by such terminology. The facts to which such statements refer are not the physical facts to which scientific theories ultimately allude. For example,

"One of the possible descriptions of the positron happens to be the time-and-charge mirror image of our usual description of the electron; this, far from pointing to a symmetry in nature, shows that our equations are ambivalent. And it cautions 
us not to mistake properties of signs

for properties of their referents.

"As can be seen, the mere awareness of the existence of metanomological statements constitutes an effective reminder that not every scientific statement refers to facts."(1961, p. 521)

Again,

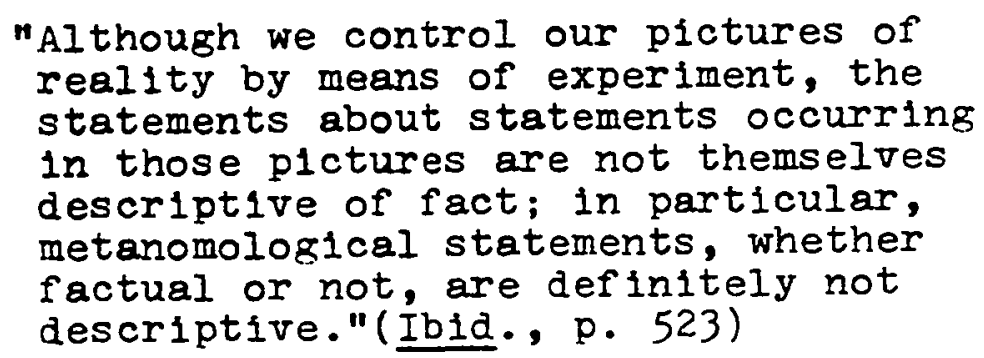

Let us now consider the distinction between

factual metanomological statements that are analytic and those that are synthetic. As an example of the analytic variety, Bunge cites the principle of CTP invariance. This, he argues, is nothing but a truth of mathematics. It is not by laboratory experiment but with the aid of paper and pencil that one determines that the equations of field theories are invariant under combined charge, time and space sign reversal. It tells us nothing about the nature of the fundamental particles to which the theory refers. Hence, one would not subject the CTP theorem to experimental test but only the law statements to which it happens to apply. Thus, while the theorem is analytic, per se, it is justifiable to regard it as factual insofar as it stands or falls with the laws $_{2}$ which are its referents. The factual metanomological statements of the 
synthetic variety may be similar in mathematical kind to those of the analytic variety. However, whereas one of the analytic variety refers to a definite theory or $l_{a} w_{2}$, those of the synthetic variety are inductive generalizations from the properties of a more or less determinate body of laws to laws in general. Thus, the simple parity principle is similar in mathematical kind to the CTP theorem. However, its use in science was as a synthetic law (or metalaw), since it is expressible in the form, 'All physical laws are invariant under space inversion.' Clearly, the hand of God would be of greater utility than that of paper and pencil in demonstrating the truth of the latter. At this point, we shall leave Bunge's analysis with a final quotation which expresses the heart of his distinction in a nutshell: he sums it up succinctly to the effect that, "...metanomological statements,... while complying with all the requisites of lawfulness do not reproduce real patterns at the conceptual level but rather describe or prescribe basic traits of law formulas." (1967b, v. I, P. 364. our italics)

We heartily agree with the last declaration. However, Bunge tends to blur its application in allowing that some metalaws may be stated linguistically as object laws and conversely. Significantly for our purposes, one of the examples which he offers is that of the principle of Classical kelativity. In the form of an object statement 
It is, 'All inertial systems are equivalent.' In the form of a metastatement it is, 'Newton's laws of motion hold In all inertial systems.' By the same token, he admits that a postulate of a theory may be dropped from the theory proper if, after appropriate rephrasing, it is introduced into the corresponding metatheory.

It is at this point that we must part company with Bunge. It is not that we disagree with his division of scientific statements into the two fundamental varieties object statement and metastatement. It is rather that we contend that the distinction needs to be sufficiently sharpened to preclude the possibility of linguistic games which permit the expression of equipolient propositions in either metastatement or object statement form, ad Iibitum. As Bunge would doubtless agree, the referents of object statements are utterly distinct from those of metastatements. The possible referents of object laws are all of the knowable properties of the objective patterns in physical reality, whereas the referents of metalaws are all of the knowable properties of laws. The latter would include their physical shape, the language in which they are expressed and, more significantly, the status which they occupy in a theory, e.s. postulate or derived theorem, and their mathematical properties, e.g. derivable from a variational principle, invariant under a certain group of transformations, etc. The point we wish 
to stress is that, speaking metaphorically, the world to which object statements refer is utterly distinct from the world to which metastatements refer; with the trivial exception that all statements,as physical entities, must obey the laws of physics.

The apparent equipollence of object statement and metastatement occurs in certain contexts where the meaning of the object statement happens to be well understood. The prototypical metastatement is of the form, "'s" is true," where $S$ is any object statement. However, in a given context an individual will either understand or fail to understand s. Clearly, if the former, then the metastatement conveys much more than if the latter be the case, but rather by association than by what is actualiy asserted in the metastatement. Consider the following metastatement: "The electromagnetic field is representable by an antisymmetric tensor of the second rank" is true." This is admittedly not a particularly rewarding example, per se, but serves admirably to illustrate our point. The metastatement is composed of everything between the double quotes. It asserts that the statement enclosed by single auotes is true, but nothing more. In other words, it is a very simple metastatement, which everyone should be capable of understanding. However, the statement enclosed by single quotes, the object statement, is fairly sophisticated. Only someone 
with a certain background of mathematical physics could be expected to understand it.

In actual fact, of course, the particular example of a metastatement which we chose would seldom occur. The object statement which is its referent, on the other hand, might very well be asserted during a lecture on electromagnetic theory. This example was deliberately chosen, however, to convey the point that the layman could understand the meaning of the metastatement, namely that the object statement has the property of being faithful to reality, just as well as the physicist. on the other hand, it is quite foreign to our intention to suggest that all metastatements are trivial. In a more serious vein, the metastatement that Newton's laws are Galilei covariant is definitely not trivial, since it conveys the information that there is a well-defined non-denumerably infinite class of spatial coordinates with respect to which the equations of motion may be formulated in a covariant manner. Notice that this is not a statement about physical reality but about the technical language which we employ to describe it. It is not equipollent to the Principle of Classical Relativity, since, among other things, the latter requires the existence of at least two frames of reference to be testable, whereas the metastatement is true independently of the existence of reference frames or anything else 
which is outside of the realm of mathematics.

Since the semantical status of the object

language is so different from that of its metalanguare,

it would probably be of some technical value to employ

entirely different symbols to distinguish laws from

metalaws. An examination of many of the treatises of

theoretical physics would surely reveal numerous examples

of the constant confounding of the two levels of discourse.

For this reason, the student of physics is sometimes apt

to be confused, not feeling certain at a particular point

in the course of a theoretical demonstration whether he

is doing physics or mathematics. However, it will suffice,

for our purposes, to keep the distinction constantly in

mind, and to resort to the familiar device of quotation

marks whenever it appears necessary.

We should perhaps add a few elementary remarks.

When a law statement is enclosed by single quotes, 'I',

the complete symbol including the quotation marks belongs to the metalanguage. It is not to be interpreted as a metalaw but as the name of a law of the object language. secondly, a metalaw must contain the name of at least one law or class of laws, e.g. 'Newton's first law', 'the laws of physics', etc. Any statement which contains the name of a law or class of laws is a metalaw statement. Consequently, no statement may belong to both the object language and the metalanguage. It would be easy to prove, 
although we omit to do so, that no object statement may be derived in the metalanguage. However, it is indeed possible to derive logical truths at each level of discourse which are isomorphic.

We have seen that it is always possible to frame an object statement $S$ in the metalanguage, the classic example being "'S' is true." We have also seen, however, that such a stratagem is specious, since it is context dependent. In particular, it depends on the knowledse of the individual to whom it is addressed. The technical language of science, however, must be context free. The meaning of a scientific statement, at any level of discourse, should have nothing to do with the politics, psychological constitution, or even the scientific training of the person to whom it is addressed. This is not a fact but the stipulation of an ideal for which the framers of scientific theories should strive.

on the basis of the foregoing considerations, we reject Bunge's claim, which on occasion he seems, himself, to reject, that an object statement may be equipollent to a metastatement, at least so far as such statements occur in scientific discourse. How, then, are we to handle the apparent instances which Bunge mentions? Since the primary function of scientific theory is the description of reality, we suggest that on methodological grounds alone it would be desirable to formulate a given 
law in the object language whenever possible. However, such a methodological rule is not, in fact, necessary if we are correct in our claim that the purported cases of equipolience are really specious. Therefore, we insist that if a statement may be formulated in the object language of science, then it is an object statement of science, tout court. It will still be useful, however, when considering the doubtful cases to investigate whether the law of interest is required as a postulate or is derivable as a theorem. Furthermore, it is important to consider whether it is deductively fertile, leading to further theorems or predictions of particular facts, or whether it may be dispensed with entirely.

So far, to make a point, we have stressed the gulf between laws and metalaws. However, the reader should not take this as an indication of the slightest disdain on the part of the writer for metalaws. On the contrary, they serve an extremely useful role in science, both heuristically and cognitively. The principle of covariance is possibly the outstanding example of the use to which metanomological statements may be put in the construction of physical theories. The role of metatheoretical analysis will be revealed to be of particular value in the field of General Relativity, which, despite its contentious status, appears to have the most richly developed metatheory of all physical science. In this case, we shall 
find the metatheory to be of the utmost heuristic value insofar as it yields theorems that are almost the analogues, formally speaking, of theorems or postulates of the object theory. Furthermore, the philosopher of physics, who takes physics, itself, as the object of study, is bound to be occupied with and learn much from the analysis of the metatheoretical structure of theories. In conclusion, we cannot resist the temptation to express the utterly extraneous wish that the practitioners of the various social sciences take heed of Bunge's distinction, which would, we strongly suspect, help them to find out what they are actually doing when they pretend to construct theories of social behaviour. 
CHAPTER IV

THE PRINCIPLE OF CLASSICAL RELATIVITY

The Principle of Classical Relativity is

applicable to several mechanical theories such as statics, rigid body dynamics, continuum mechanics, etc. However, it will suffice for our limited purposes to use only the elementary theory of point mechanics by way of illustration. Furthermore, we shall not attempt a rigorous axiomatization of this theory which, again, is not necessary for our limited objectives. However, the reader is advised to consult Bunge(1967a) for such an axiomatization. The reader should keep in mind that our purpose is neither to provide a compendium of physical theories nor, still less, to provide an axiomatization of the basic theories of physics, but merely to discuss one crucial problem which is connected with the foundations of all relativistic theories, in the broadest sense of the term relativity. From the standpoint of the worker in the axiomatic foundations of physical theories, we hope, this work of ours should be a useful prolegomenon.

We propose to begin with a straightforward and elementary discussion of the covariance properties of the physical theory of interest and then to deal with the Frinciple of Classical Relativity. We shall then proceed to take up the elementary theory of polnt mechanics once 
more but for the purpose of reaching one non-elementary result, namely of showing that the usual geometrical background may be dispensed with in favour of the less restrictive presuppositions of affine geometry. But let us now begin at kindergarten which, in this case, is simply to state Newton's three laws of motion for a system of point mechanics.

I In the absence of external forces, the equations of motion of a particle are:

$$
m \ddot{X}=0, m \ddot{Y}=0, m \ddot{Z}=0,
$$

or more succinctly, $m \ddot{X}^{\dot{I}}=0 \quad(1=1,2,3)$

II The motion of the ith. particle due to external and internal forces is given by:

$$
\sum_{j} \vec{F}_{j i}+F_{i}^{(e)}=m \ddot{X}^{i}=m \vec{a}
$$

III The internal force between the ith. and jth. particle is such that:

$$
\vec{F}_{i j}=-\vec{F}_{j i}
$$

These three postulates were regarded by Newton as the axiomatic basis of the object theory of elementary point mechanics. However, as has so of ten been pointed out, they lack physical content as they stand, quite apart from the fact that the actual differential equations of motion of a system of point mechanics could not be set up and solved without an explicit formulation of the force functions, viz. $F\left(X^{i}, \dot{x}^{1}, t, \ldots\right)=m \ddot{x}^{1}$. We refer, of course, to the lack of a frame of reference with respect to which these laws are supposed to hold. 
Accordingly, we add the additional axiom that there exists a frame of reference $K$ such that I, II, III hold with respect to $i t$. It should be noted that the $x^{i}$ refer to the physical coordinates of the point particles, which automatically puts the above axioms in the object language. We now turn immediately to the covariance properties of the fundamental laws: I, II, III. The elementary treatments of Newtonian Mechanics generally Iimit the discussion of the latter's covariance properties to the special, homogeneous Galilean group of transformations. That is to say, given a system $S$ of Cartesian coordinates $x^{i}$ and a time scale for $t$ with respect to which Newton's laws have the standard form, they will retain that form when expressed in terms of a second system $\overline{\mathrm{S}}$ with coordinate variables $\overline{\mathrm{x}}^{1}$, so chosen that the origin of $\bar{s}$ has the coordinate values $x^{i}=\bar{x}^{1}=0$ at $t=t_{0}=\bar{t}_{0}$, and are undergoing a change given by $\delta \bar{x}^{1}=\frac{d x^{\prime}}{d t}=\vec{V}=$ const., $\delta \bar{x}^{2}=\frac{\partial \bar{x}^{2}}{\partial x^{j}} d x^{j}=0, \delta \bar{x}^{3}=\frac{\partial \bar{x}^{3}}{\partial x^{j}} d x^{j}=0$. In words, at an arbitrary initial time, the origins of the two systems coincide while the variable $\bar{x}^{1}$ of $\bar{s}$ is undergoing a constant and continuous change of value with respect to $x^{1}$, and the variables $\bar{x}^{-1}$ have fixed values relative to $x^{2}$ and $x^{3}$. The values of the $\bar{x}^{i}$ are given in terms of the $x^{i}$ by the set of transformations: 


$$
\begin{aligned}
& x^{1} \rightarrow \bar{x}^{-1}=x^{1}-\vec{v} t \\
& x^{2} \rightarrow \bar{x}^{2}=x^{2} \\
& x^{3} \rightarrow \bar{x}^{3}=x^{3} \\
& t \rightarrow \bar{t}=t
\end{aligned}
$$

It is obvious that the mappings (4.4) form a group: the special, homogeneous Galilean group, which we shall label $G_{S}$.

In the more general case, however, each of the $\bar{x}^{1}$ will be undergoing a change of value with respect to all of the $x^{i}$. Nevertheless, any such relationship between two coordinate systems which preserves the form of Newton's laws may be made equivalent to the special Galilean relationship by a suitable transformation $\bar{S} \rightarrow \bar{s}$ in the form of a rotation combined with a change of origin. We call the latter transformation the inhomogeneous rotation group in three dimensions and use the label $\mathrm{R}_{13}$ : That $R_{13}$ is a group is as obvious as the case of $G_{S}$. It is easily shown that the direct product $R_{i 3} \times G_{s}$ is also a group of transformations.

Let us now apply these considerations to Newton's laws of motion. To signify that we are in the realm of metatheory, we shall employ the two notational devices of enclosing the statement of laws in single auotes and the more elegant one of employing lower case letters for coordinate variables as distinct from upper case letters for the physical coordinates of point particles. 
We begin by noting that since the special

Galilean transformation amounts to the addition or subtraction of a factor which is the product of a constant first derivative and the first power of the independent variable, any second or higher order derivatives will be unaffected by a transformation which is an element of $G_{S}$. The simplest general expressions which are covariant with respect to $G_{S}$ are, therefore, $k \frac{d^{2} x^{i}}{d t^{2}}=0$, where $k$ is any constant. Thus, a necessary condition for the covariance of 'Newton I' is that mass be a scalar quantity. Although the content of the preceding paragraph is almost childishly simple, it serves to convey the following useful philosophical message. The metatheoretical analysis of the Galilean group of transformations was devoid of physical content. It dealt only with the mathematical relationships holding between the coordinate variables of an infinite set of Cartesian coordinate systems under the condition of the covariance of a class of lawlike formulae. Nevertheless, these considerations gave rise to a result which, while not qualifying as a 'fact of naiure', had definite implications concerning the formulation of an object law, namely that if 'Newton I' is correct then the mass of a particle must be a scalar quantity. 'Ihus, we are obljged to add the further axiom to the effect that mass is a constant function from point particles into the real ine. In other words, this frament 
of metatheory decreed that whatever form our physical laws happen to take, the quantities which we employ to represent them must be 'well-behaved' geometrical objects; and it is the metatheory which yields the appropriate rules of behaviour. In this sense, it may be regarded as a type of syntax, which, like the syntax of ordinary English, at least until recently, cannot single out true sentences but can certainly reject non-sentences. However, as we proceed, we shall find that such an analogy is not the strongest possible one, since it tends to underplay the remarkable heuristic power of metatheoretical analysis. 'Turning now to Newton's second law, let us tentatively combine the internal and external forces acting on the ith. particle and rewrite it in the simple form:

$$
\sum_{j} F_{j}=\sum_{j} m_{j} \ddot{x}^{1}
$$

We have already determined that $m$ is a scalar and that $\ddot{x} \dot{I} \rightarrow \ddot{\bar{x}}^{i}=\ddot{x}^{i}$ under $G_{S}$. Consequently, the magnitude of $F$ must be preserved under $G_{S}$ in order that the equality expressed by 'Newton II' be preserved. But since F cannot be a scalar, the only object at our disposal is the three-vector. We conclude that a necessary condition for 'Newton II' is that force be represented by the type of geometrical object that we have called a vector. Since we have restricted the treatment to Cartesian coordinate systems, the distinction between contravariant and 
covariant vectors is superfluous.

To determine whether 'Newton II' is covariant,

under the seneral group of Galilean transformations $R_{13} \times G_{S}$, we must now investigate the group $\mathrm{R}_{i 3}$ or simply $\mathrm{R}_{3}$, the group of homogeneous proper rotations. It is an elementary theorem of mathematics that the principal invariant of $\mathrm{R}_{3}$ is that of coordinate distance. Thus, under the group $\mathrm{R}_{3}$, we may write the equality:

$$
\left(x_{i}-x_{j}\right)=\left(\bar{x}_{i}-\bar{x}_{j}\right)
$$

More generally, if $c_{i k}$ is the rotation matrix, then:

$$
\begin{gathered}
(d s)^{2}=\delta_{i k} d x^{i} d x^{k}=\delta_{i k} c_{i k} d x^{i} d x^{k}=\delta_{i k} d \bar{x}^{i} d \bar{x}^{k}=(d \bar{s})^{2} \quad(4.7) \\
\text { It is assumed that the interparticle forces }
\end{gathered}
$$
are a function of the straight line distance between the point particles. Consequently, they will not be affected in magnitude by a coordinate rotation. Furthermore, since the $c_{1 k}$ may be interpreted as the direction cosines between the coordinate axes, and the cosine is an even function, we are assured of the covariance of 'Newton III' under the group of spatial rotations $R_{3}$ and, intuitively without proof, under $\mathrm{R}_{i 3}$. Hence, 'Newton III' is covariant under the general Galilean group of transformations. So far, our main achievement has been to discuss informally the covariance properties of Newton's equations of motion without any reference to the Principle of Classical Relativity. This has served to illustrate Bunge's point that the determination of the covariance properties 
of a given theory is, indeed, a paper and pencil operation. But it has served the additional purpose of showing that the concepts of relativity and covariance are not synonymous but, in fact, belong to different levels of scientific discourse. Let us now turn to a consideration of the Principle of Classical Relativity. At the risk of seeming otiose, we wish, as a preliminary, to impress on the reader the distinction, which we have already lald bare in Chapter II, between coordinate transformations and frame transformations. Further, in elucidating the concept of relativity, we alluded to the physical content of law statements as distinct from their mere formal appearance. A perlisal of the literature of physics has convinced us that the distinction is ambiguous and fails to be grasped by many physicists. We may bring out this distinction more forcibly by remarking that it is by no means inconceivable that the physical content of a law could only be preserved under a given frame transformation by an alteration of its form while, conversely, its form could only be preserved by an alteration of its content. Whether this will actually occur will depend, in most cases, on the geometrical objects which are at our disposal for the formulation of such laws. We shall support this crucial point with a straightforward example from elementary point mechanics with its standard mathematical 
apparatus.

We consider the motion of a system of point particles with respect to an arbitrary frame of reference with origin 0 . The total angular momentum about 0 is given by:

$$
\vec{L}=\sum_{i} \vec{r}_{i} \times{\overrightarrow{p_{i}}}_{i}
$$

Now we signify the radius vector of the centre of mass of the system of particles by $\vec{R}$ and let $\overrightarrow{\vec{r}}_{i}$ be the radius vector from the centre of mass to the ith. particle. Ne then form the obvious frame transformation equations between the arbitrary system and the centre of mass system:

$$
\left.\begin{array}{l}
\vec{r}_{i}=\vec{r}_{i}+\vec{R} \\
\vec{v}_{i}=\vec{v}_{i}+\vec{v}
\end{array}\right\}
$$

We then rewrite $(4.8)$ as:

$$
\begin{aligned}
\vec{L} & =\sum_{i}\left(\vec{r}_{i}+\vec{R}\right) \times \sum_{i} \frac{d}{d t}\left[m_{i}\left(\vec{r}_{i}+\vec{R}\right)\right] \\
\text { or, } \vec{L} & =\sum_{i}\left(\vec{r}_{i}+\vec{R}\right) \times \sum_{i}\left(\frac{d}{d t} m_{i} \overrightarrow{\vec{r}}_{i}+m_{i} \vec{v}\right)
\end{aligned}
$$

Expanding and rearranging the terms of (4.11) we get:

$$
\vec{L}=\sum_{i} \vec{r}_{i} \times m_{i} \vec{v}_{i}+\sum_{i} m_{i} \overrightarrow{\vec{r}}_{i} \times \vec{v}+\vec{R} \times \frac{d}{d t} \sum_{i} m_{i} \overrightarrow{\vec{r}}_{i}+\vec{R} \times \sum_{i} m_{i} \vec{v} \quad \text { (4.12) }
$$

But $m_{i} \vec{r}_{i}$ is the radius vector of the centre of mass in the centre of mass frame of reference and must, therefore, vanish. Hence, (4.12) becomes:

$$
\vec{L}=\vec{R} \times M \vec{v}+\sum_{i} \overrightarrow{\vec{r}}_{i} \times{\overrightarrow{\vec{P}_{i}}}
$$

(4.13) is the expression for the angular momentum of the system of particles about the original origin 0 , when referred to the centre of mass coordinates. The second term on the right hand side of (4.13) is identical in 
form to the right hand side of the original expression (4.8). The first term on the right hand side of (4.12) is the angular momentum of the centre of mass with respect to the origin of the original coordinate system. Finally, since $\vec{R}$ is arbitrary, we may set it equal to zero and take the centre of mass as our new origin. Our new expression is then:

$$
\overrightarrow{\bar{L}}=\sum_{i} \overrightarrow{\vec{r}}_{i} \times \overrightarrow{\bar{D}}_{i}
$$

Now we wish to draw the reader's attention to the comparison of both (4.13) and (4.14) with (4.8). (4.13) is a more complex expression than (4.8) which results from a new coordinatization of the numerically identical frame of reference. It has the same physical content as (4.8) but a different form. On the other hand, $(4.14)$ has precisely the same form as (4.8) but a different physical content. Whereas (4.8) expresses the angular momentum of a system of point particles about an arbitrary point, (4.14) expresses the angular momentum of the same system of point particles about a particular point, namely the centre of mass of the system of particles. By now, the point has been sufficiently laboured, and we turn directly to the Principle of Classical Relativity.

In studying our formulation of the Principle of Classical Relativity, the reader should first refer to the schema of Chapter II, sec. 7. Accordingly, we formulate the principle as follows: 
$\left\{I_{n}\right\} \equiv\left\{I_{n 1}, I_{n 2}, I_{n}\right\}$ is the set of axioms of Newtonian Point Mechanics.

$\{K\}$ is the set of possible frames of reference.

$\{T\}$ is the set of possible frame transformations modelled by elements of the transformation group $G_{S}$.

For any $I_{n} \in\left\{L_{n}\right\}$ and any $t \in\{T\}$, there exist at least two distinet $k_{i}, k_{j} \in\{k\}$, such that: $I_{n}$ w.r.t. $k_{i} \in\{k\}$ $\leftrightarrow t\left(I_{n}\right)=s . \bar{I}_{n}$ w.r.t. $k_{j} \in\{K\}$. When we interpret $\{K\}$ as the set of possible frames of reference, we intend to imply only technical feasibility. Thus, it is feasible to use a laboratory or a galaxy as a frame of reference but not a leaf falling, from a tree. On the other hand, when we interpret $\{T\}$ as "the set of possible frame transformations...", we rule out all those elements of the Galilean group which correspond to mere coordinate transformations. Wiost of the standard formulations of the Principle of Classical Relativity are high level theorems which follow from our own formulation. For example, it is sometimes stated in the form of a so-called 'principle of impotence', viz. 'It is impossible to detect an absolute velocity by mechanical means.' This is true but not especially enlightening, particularly for the mathematician. Fossibly the worst version of all is, 'All inertial frames are equivalent.' The latter is not only vague but also viciously circular.

The reader has doubtlessly already noted that
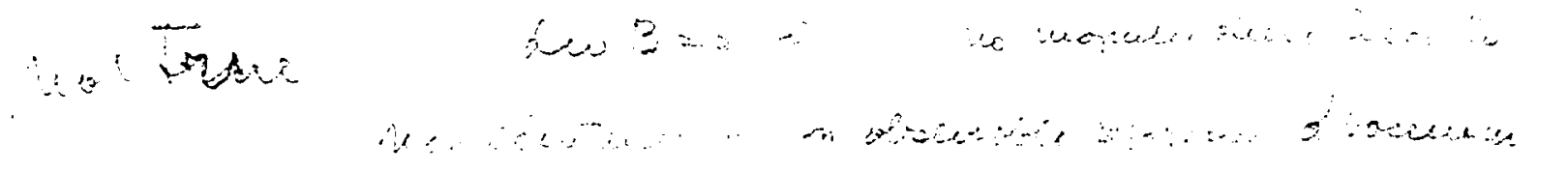
our formulation omits any explicit reference to the set $\{I\}$ of inertial frames. In fact, the concept is implicit in the above formulation, since the set of inertial frames $\{I\} \subset\{K\}$ is precisely the subset of frames of reference for which the Classical Principle holds. The advantage of our avoiding explicit reference to the set of inertial frames is that it precludes the possibility of misjudging the nature of the concept. Furthermore, it is an experimental fact that every $i_{j} \in\{I\}$ differs from every distinct $i_{k} \in\{I\}$ by a constant velocity. However, it would be possible, albeit redundant, to replace the $k_{j}$ of our principle by $i_{j} \in\{I\}$, if the reader should experience any discomfort in contemplating a formulation of a restricted principle of relativity which contains no explicit reference to an inertial frame. Our own particular stratagem does, however, have the virtue of achieving the maximum of logical generality.

An important point vis à vis the Classical Principle, whose significance will emerge in later chapters, is the following. The Galilei covariant theories are, in general, those which are broadly called mechanical. Crudely speaking, they pertain to the kinematics and dynamics of bits of matter in its various forms. Now a reference frame is preeminentiy a chunk of matter and, therefore, subject to the laws which describe the behaviour of such chunks. Consequently, the kinematical relationships 
between frames of reference and, in particular, inertial frames must be Galilei covariant. We may contrast this situation with that of the theory of electrodynamics. On the one hand, we are interested in the transformation properties of the field equations when referred to different frames of reference. But changing fields cannot serve as reference frames. Thus, there is a fundamental diversity between the principal subject matter of the theory and the frames of reference such as radar stations which are necessary to describe and to test its laws. The absence of such diversity in the case of elementary mechanics, including 'action at a distance' gravitational theory, is an obvious ground for the confusion which has existed between the covariance properties of the formal laws and the appropriate relativity principle. We have frequently alluded to the formal character of covariance principles in contrast to the factuality of relativity principles. However, apart from making the rather obvious point that frames of reference belong to the physical world, we have said little in justification of the second part of our thesis which will, however, gradually emerge in the course of this dissertation. Nevertheless, a simple example should serve temporarily to overcome this ommision. On the basis of the argument of the preceding paragraph, let us imagine two frames of reference which are in uniform parallel motion with respect 
to each other. At an arbitrary initial time $t_{0}$, a molecule of one frame located on the $X$-axis coincides with a molecule located on the $\bar{x}$-axis of the second frame. on the basis of the first of the equations (4.4), assuming a molecule to be a point particle, the kinematical relationship between the physical coordinates of the two molecules is given by:

$$
\bar{x}^{1}=x^{1}-\overrightarrow{v t}
$$

From (4.15), by differentiation, we obtain:

$$
\frac{d \bar{X}^{\prime}}{d t}=\frac{d x^{\prime}}{d t}-\vec{V}
$$

which is the well-known Galilean law of the composition of velocities. $(4.16)$ is an immediate consequence of the Principle of Classical Relativity and is, moreover, factual. It is tempting to regard $(4.16)$ as a law of arithmetic but this, again, we suspect is due to the special character of Newtonian Mechanics. Should the reader doubt the physical content of the law of the composition of velocities, let him consider the case of a bullet which leaves a rifle with a muzzle velocity $\vec{V}$ and travels with a velocity $\vec{V}+\vec{W}$ with respect to the earth, where $\vec{W}$ is the velocity of the rifle with respect to the earth. The latter certainly satisfies a sufficient condition of factuality, viz. it is both logically consistent and false, i.e. an approximation of the purportedly true law of velocity composition of special Relativity physics. In fine, it is a claim about the physical union of two velocities, 
which is quite different from a formal proposition about the addition of two numbers(Cf. Bunge, 1967a, p. 110f.). In accordance with the position which we upheld in Chapter III, since the Frinciple of Classical Relativity entails an object law of physics, it must belong to the object theory. Wany other examples abound of the factual consequences of Classical Relativity. We merely selected the simplest and most direct for the sake of brevity. (The philosophical reader is advised to gloss over the following part of this chapter and to return to it after he has read Chapter VIII.) In the preceding discussion of Newtonian Mechanics, we took for granted the usual geometrical background of that theory as presented in the various classical treatises, namely that the Euclidean metric is globally applicable to physical space and that physical time has the metric of a Euclidean straight Iine. For both cognitive and pedagogical reasons, it is instructive to develop the theory on the basis of the less restricted background of affine geometry. Among other things, this will serve to bring into sharper relief the distinction between the formalism and the physical content of a theory. However, since this is primarily a philosophical dissertation, it will suffice to treat a tiny fragment of Newtonian Mechanics, namely Newton's first law of motion for a point particle. The mathematical justification for the following development 
is to be found in schrodinger (1950, Ch. 5,6 \& 7 , passim). Our primitive background will be the usual simply-connected, differentiable space-time manifold, $V_{4}$. We shall endeavour to show how such a manifold may be coordinatized independently of the standard Euclidean metric. we first consider a non-denumerably infinite family of non-intersecting hypersurfaces of $V_{4}$ which are characterized by a single parameter $t=t\left(x^{\mu}\right) .(\mu=0,1,2,3)$ Intuitively, for any $t=$ const. hypersurface, we have a subspace in which every point is connected to every other point by the relationship of simultaneity. Thus, the parameter $t$ corresponds to the absolute time of Newton. We now introduce a three-parameter congruence of curves with the single restriction that each curve of the congruence will intersect a given hypersurface of simultaneity at one and only one point. It follows that no two points on a given curve may be simultaneous. Thus, $t$ may serve as a path parameter for the curves of the congruence. Intuitively, each curve represents a spatial point which endures through time. The entire congruence is the counterpart of Newton's absolute space. Any spatial point will be given by the equation:

$$
s^{i}=s^{i}\left(x^{\mu}\right)
$$

We may now coordinatize the manifold by choosing coordinates such that $t=x^{0}$ and $s^{i}=x^{i}$. Thus, we may write:

$$
x^{\mu}=x^{\mu}\left(s^{i}, t\right)
$$


We now construct a field of normals to the hypersurfaces given by:

$$
N_{\mu}=\frac{\partial t}{\partial x^{\mu}}
$$

and $a$ field of tangents to the curves of the congruence given by:

$$
u^{\mu}=\frac{\partial x^{\mu}}{\partial t}
$$

It is obvious that at any point of the manifold, the relationship between the normal field and the tangent field will be given by:

$$
\mathrm{N}_{\boldsymbol{\mu}} \mathrm{U}^{\mu}=1
$$

In particular, with the appropriate coordinatization, there would always be possible a mapping such that at any point:

$$
\begin{aligned}
& N_{\mu}=(1,0,0,0) \\
& U^{\mu}=(1,0,0,0)
\end{aligned}
$$

We shall now, merely as an aid to intultion, make a brief mathematical detour by introducing a metric form as a temporary scaffolding. We define a temporal interval between the neighbouring points $x^{\mu}$ and $x^{\mu}+d x^{\mu}$ by :

$$
(d r)^{2}=N_{\mu} N_{v} d x^{\mu} d x^{\nu}
$$

It may be shown by standard mathematical means that the field of normals $N_{\mu}$ must be the gradient of a scalar field. Consequently, the above interval is integrable, and a finite time interval may be given by the difference between the two numbers characterizing the values of the 
parameter $t$ associated with two distinct hypersurfaces. In the same way, we introduce a spatial metric for two neighbouring curves of the congruence, $s^{i}$ and $s^{i}+d s^{i}$ (or $\mathrm{x}^{i}+d \mathrm{x}^{i}$ ). We then introduce the quadratic form which is equivalent to a locally Euclidean metric:

$$
(d s)^{2}=\delta_{i j} d s^{i} d s^{j}=\delta_{i j} d x^{i} d x^{j}
$$

This, again, can be shown to be integrable by standard mathematics. Given two neighbouring points of the manifold, we have:

$$
d s^{i}=\frac{\partial s^{i}}{\partial x^{\mu}} d x^{\mu}
$$

Now we introduce by definition:

$$
S_{\mu v}=d f \cdot \delta_{i j} \frac{\partial s^{i}}{\partial x^{\mu}} \frac{\partial s^{j}}{\partial x^{v}}
$$

$(4.25)$ and (4.26) enable us to write (4.24) in the form:

$$
(d s)^{2}=s_{\mu \nu} d x^{\mu} d x^{2}
$$

With the appropriate coordinatization, $S_{\mu r}=\operatorname{diag}(0,1,1,1)$, a result which we shall use in Chapter VIII.

$$
\text { we now return to our main argument. In place }
$$

of (4.27), which is a disguised Newtonian space-time metric, we are able to introduce its equivalent without reference to the familiar parameters $t$ and $s^{i}$. We impose on the manifold a flat, symmetric affinity which is, in general, almost arbitrary, such that under the conditions in which $N_{\mu}$ and $U^{\mu}$ would have components $(1,0,0,0)$, the affinity vanishes identically. The reader should note that an affinity behaves like a tensor under linear or affine transformations. That is to say, its transformation law 
is linear and homogeneous. On our affine manifold, the constancy of the $U^{\mu}$ and the $N_{\mu}$ is a covariant property. That is to say, their covariant derivatives vanish identically. On the basis of the choice of a fourdimensional Cartesian coordinate system, we have at our disposal the following geometrical objects: $N_{\mu}=U^{\mu}=(1,0,0,0)$ and $\Gamma_{\mu v}^{p}=0$. We now proceed to reconstruct Newton's first law. This will be followed by a further discussion of the Principle of Classical Relativity.

The physical coordinates of a particle may be represented by means of the path parameter $s$, namely:

$$
x^{\mu}=f^{\mu}(s)
$$

(4.28) are the equations for all of the trajectories of a particle that are kinematically conceivable. However, It is kinematically impossible for two points on the trajectory of a particle to be simultaneous, so that the path parameter could be time as measured by a standard clock. The velocity of the particle would be given by $\frac{d f^{*}}{d t}$ and would satisfy:

$$
\begin{aligned}
& N_{\mu}{\frac{d f^{M}}{d t}}^{M}=1 \\
& \text { In our system, } s \text {, or we may say } t \text {, is the }
\end{aligned}
$$
path parameter of an affine geodesic on the manifold given by $\Gamma_{\mu v}^{p}=0$. It should be noted that without recourse to metrical geometry it is possible to compare distances so long as they are on the same geodesic curve. The affinity is only defined up to a linear transformation with 
constant coefficients, so that one could not compare distances along two distinct geodesic curves. It should also be noted, for future reference, that the condition for the vanishing of the flat affinity is the vanishing

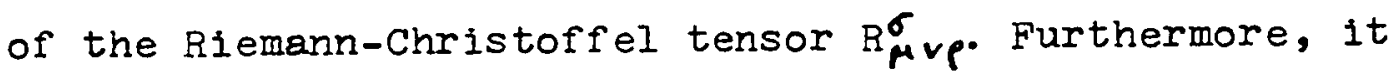
is shown in most of the standard texts that the RiemannChristoffel tensor is defined wholly in terms of affine symbols. The metric term $g_{\mu \nu}$ is only required for the formation of the completely covariant form of the RiemannChristoffel tensor. We merely mention this to preclude the reader's supposing that we have smuggled metrical geometry through the back door. This is a reasonable suspicion on the part of the reader who has been exposed to less rigorous treatments of manifold geometry or tensor analysis in which the affine basis is often disregarded and the Riemann-Christoffel tensor is defined in terms of the metric coefficients and their derivatives, i.e. the Christoffel symbols.

We are now prepared to write the equations of motion of a free particle in generally covariant form. The equations of motion of a point particle under the influence of no forces, corresponding to 'Newton $I$ ' are:

$$
\frac{\delta^{2} f^{M}}{\delta t^{2}}=\frac{d^{2} f^{M}}{d t^{2}}+\Gamma_{\rho^{\sigma}}^{\mu} \frac{d f^{\mu}}{d t} \frac{d f^{\sigma}}{d t}=0
$$

The most significant property of an affinity is that it is always possible to find a permissible mapping in which the components of the affinity vanish identically. We may 
single out the equivalence class of coordinate systems in which the affinity vanishes, and in terms of such coordinates rewrite $(4.30)$ in the form:

$$
\frac{\delta^{2} f^{M}}{\delta t^{2}}=\frac{d^{2} f^{M}}{d t^{2}}=0
$$

Integrating twice, we get:

$$
f^{\mu}=c^{\mu} t+k^{\mu}
$$

We may relate (4.32) to the equations of motion of a free particle in an inertial frame of reference by choosing a real clock with a time scale such that $c^{0}=1$ and $\mathrm{x}^{0}=0$. Then the $\mathrm{c}^{1}$ are the familiar components of the velocity of the point particle.

once again, we note that even following the choice of an equivalence class of coordinate systems in which the affinity vanishes, which are quite properly described as geodesic systems, there does not result a set of coordinate systems which is coextensive with the set of inertial frames of reference. When we speak of a reference frame in the context of four-dimensional spacetime, we refer not only to a physical scaffolding of some sort but also to a standard clock.

Conversely, we may choose coordinate systems in which the components of the affinity do not vanish identically. The particle trajectory would continue to be classifiable as free in accordance with (4.30), and certain of these coordinate systems would again serve as models of reference frames. However, one would now have 
to take account of inertial forces. In fine, we come to the same conclusion that we reached in dealing with Newtonian Mechanics from a completely elementary standpoint. The inertial forces are characteristic of theories that are associated with restricted principles of relativity. However, we have learned a new feature of the metatheoretical background of Newtonian Mechanics, namely that the standard Euclidean metric has no intrinsic significance for Newtonian Mechanics since it may be replaced by the less restrictive affine geometry. This, in itself, is an example of a significant proposition of the metatheory of Newtonian Mechanics. We refrain from following this development any further since it would involve the use of advanced mathematical techniques which we prefer to introduce gradually.

Insofar as the covariance group of a set of space-time equations is determined in part by the background geometry of the theory, it is clear that the covariance properties of a theory, per se, have no physical content. The covariance group of (4.30) is the most general real linear group in four dimensions. However, while the Galilean group is a subgroup of the fourdimensional affine group, it would be a logical mistake to modify the Principle of Classical Relativity on account of the preceding considerations. We suggest, therefore, 
that it would be useful to make a methodological distinction between the covariance group of a physical theory and the relativity group of the same theory. On this basis, instead of declaring Newtonian Mechanics to be Galilei covariant, which is an understatement, we would more aptly describe it as Galilei relativistic. The covariance group of elementary point mechanics is actually the direct product of three groups, namely the inhomogeneous, proper rotation group, the special Galilean group and the group of time translations: $R_{13} \times G_{S} \times T$. From the time of Newton until that of Einstein, $R_{13} \times T$ was regarded as expressing the universal symmetries of all physical theories. That is to say, space and time were regarded as absolutes. On the other hand, $G_{s}$ was regarded as the expression of the particular properties of Newton's laws of motion. Since $R_{13} \times T$ was the covariance group of all theories, it did not play any role in the formulation of the Principle of Classical Relativity. In semi-popular expositions, the interpretation of $R_{13} \times T$ is to the effect that if an experiment be performed at a certain place and at a particular time with apparatus which has a specific spatial orientation, then, ceteris paribus, the 'identical' experiment performed at a different place, a different time, and with a different spatial orientation would yield the identical result, i.e., from a physical standpoint, space is homogeneous and 
isotropic and time is homogeneous. While the foregoing is an example of a pedagogical fiction, it serves to iliustrate the outlook of the Newtonian era. On the other hand, the general Galilean group was obviously regarded as a specific property of Newtonian Mechanics. In fact, it is this group alone that is singled out in most textbooks as the covariance group of the theory; and the corresponding relativity principle is formulated in connection with the latter group alone. While the thinking is often confused, we are now in a position to offer some rational justification for the distinction.

$\mathrm{R}_{13} \times \mathrm{T}$, while a covariance group of Newtonian Wiechanics, is interpretable only in terms of alternative coordinatizations of the physical system of interest. on the other hand, $G_{S}$ is interpretable as the symmetry group of the equivalence class of inertial frames of reference. In our exposition of the metatheoretical background of Newtonian Mechanics, we introduced the vector $U^{\mu}$. This, of course, is directly related to the velocity $\frac{d x^{\mu}}{d t}$ of the object theory. Experiment revealed that $\frac{d x^{n}}{d t}=V^{\mu}$ is not an observable property, i.e. it is not an absolute like spatial distance. Since $U^{\mu}$ is not, in the foregoing sense, an absolute, it follows that there must be a relativity principle, namely that the velocity of a material object (any object under the purview of Classical Mechanics, which includes frames of reference) cannot be ascertained. 
In conclusion, we may summarize our argument that the covariance group of Newtonian liechanics, in its elementary form is $\mathrm{R}_{13} \times \mathrm{G}_{\mathrm{S}} \times \mathrm{T}$. On the assumption of an affine as distinct from a metric manifold, the covariance group of Newtonian Mechanics is the affine group in four dimensions. It is clear that both of these declarations pertain to the mathematical formalism of the theory. The Principle of Classical Relativity, to the effect that the inertial frames of Newtonian Mechanics are defined up to a Galilean transformation is an experimentally confirmed hypothesis which could, we suspect, be shown to serve as part of an alternative axiomatic basis for Newtonian Mechanics.

In passing, it may be mentioned that a rather conclusive piece of intuitive support for the essential distinction between the covariance group and the relativity group of Newtonian Mechanics is that $T$, the group of time translations, is a subgroup of the covariance group. But that is to say that the laws of Newtonian Mechanics have a formal structure which is invariant under time reversal, since every element of a group must have an inverse. But it does not follow from this that it is possible to find a frame of reference in which the order of physical events is reversed. Hence, $T$ cannot be a subgroup of the relativity group of Newtonian Mechanics. 


\section{CHAPTER V \\ SPECIAL RELATIVITY KINEMATICS}

It is customary to develop introductory expositions of special Relativity on the basis of the well-know kinematics of that theory. The shortcomings of this tradition are: (i) that it gives a misleading flavour to the theory by depriving it of most of its physical content; (ii) that it gives a superficial appearance of arbitrariness to the theory in the eyes of the student; (iii) that it leads the amateur, who often reads only the introductory chapters of textbooks on physics, to suppose that Special Relativity is nothing but a theory of kinematics; (iv) that, in particilar, it suggests to the philosopher that the Lorentz transformation is of purely kinematical significance, which has led many philosophers and, curiously, even eminent physicists such as Eddington, to suppose that it is a theory about the way in which we observe the world and, hence, subjectivistic. Experts in the foundations of physics, such as Bunge, argue, accordingly, that the theory should be developed on the basis of the structure of electrodynamics, which, logically speaking, is its legitimate parent. However, keeping such shortcomings in mind, we shall follow the traditional procedure of beginning with the kinematical part of the theory in virtue of its relative 
simplicity, which enables us to clarify its logical

structure and, in particular, to distinguish the object theory from the metatheory, which is a rather subtle problem in this context, while avoiding the additional mathematical and physical burdens of the rather complex theory of electrodynamics. Nevertheless, in deference to the demands of logical rigour, we shall refrain from making a formal statement of the Principle of special Relativity until we come to the chapter of this dissertation which is devoted to electrodynamics. We should also add that what folluws does not pretend to be an exhaustive treatment of special Relativity Kinematics. For example, the reader will find no reference to the relativistic Doppler effect, while the Lorentz-Fitzgerald contraction will simply be taken for granted. There are many excellent textbooks on the subject(e.8. Møller, 1952), and the interested lay reader should consult one of these for further information or read the appropriate section of Bunge(1967a).

The fundamental postulate of special Relativity Kinematics is the law of the constancy of the velocity of light. There is an abundance of experimental evidence in support of the factual hypothesis which asserts that, in an inertial frame of reference, in vacuo, the ratio between the distance traversed by a light ray travelling along a given path and the total time elapsed is equal to a 
universal constant c. However, we prefer to say that an inertial frame of special Relativity is one in which $c$ is a universal constant. In other words, we treat the law of Iight propagation as the fundamental law which is the basis for the selection of the equivalence class of inertial fremes of that theory. For the time being, at least, we may define an inertial frame of special Relativity physics as one in which the form of the law of light propagation contains no terms which depend essentially on the state of motion of the given frame. Our primary purpose in this chapter is to show that the principle of relativity which emerges from this physical hypothesis is, indeed, an object statement of the theory. In other words, in the sense of the preceding chapter, we shall argue that one is fully justified in speaking of Lorentz relativistic as distinct from Lorentz covariant theories. We also intend to shed some much needed light on the distinction between a so-called Galilean frame of reference and what we shall call a Lorentz frame of reference. Were we to follow the procedure of the previous chapter, we would begin with the equations of a relativistic theory and proceed to determine their covariance properties. However, since we have elected to follow a pedagogical route rather than a logical one, we shall begin by deriving the Lorentz transformation from a simple example. Our procedure is deliberately calculated to reveal the 
assumptions on which such a derivation may be based. We consider two inertial frames of reference, $K$ and $\bar{K}$, modelled by Cartesian coordinate systems, $S$ and $\bar{S}$, which are so oriented that their axes are parallel and their respective origins, 0 and $\bar{o}$, coincide at $t=\bar{t}=0$. In other words, we shall begin with the situation which corresponds to the restricted, homogeneous transformation of Newtonian Mechanics. Incidentally, we shall now use the term restricted in a different sense from that in which it is usually employed in the theory of groups of transformations. In that theory, restricted is taken to mean the exclusion of improper rotations, i.e. With a determinant equal to -1 . Such inversions, while interesting, play no significant role in any of the theories which we discuss in this dissertation. While the group of spatial inversions may be a covariance group of these theories, it is definitely not a relativity group of any of them since, apart from obvious grounds of intuition, it is non-continuous. Accordingly, we shall use the useful term restricted to apply to the case where the motion of one frame of reference with respect to a 'stationary' frame may be represented by a vector of which only one component differs from zero. Where all three components are generally non-vanishing, we shall employ the term general. From the principle of constancy of light propagation, it follows that the equation for a spherical 
wave front in $K$ emitted from $O$ has the form:

$$
x^{2}+y^{2}+z^{2}=c^{2} t^{2}
$$

$\bar{K}$ is, ex hypothesi, also an inertial frame. Accordingly, the form of the wave front equation must, with respect to $\overline{\mathrm{K}}$, be:

$$
\bar{x}^{2}+\bar{y}^{2}+\bar{z}^{2}=c^{2} E^{2}
$$

We first assure ourselves that the mapping between $S$ and $\bar{S}$ is not given by the restricted, homogeneous Galilean transformation (4.4). Performing the mapping (4.4) on (5.1) yields:

$$
x^{2}-2 x v t+v^{2} t^{2}+y^{2}+z^{2}=c^{2} t^{2}
$$

It is obvious that $(5.3)$ is inconsistent with $(5.1)$. Consequently, we make our first metatheoretical declaration, albeit negative, that 'the wave front equation' is not Galilei covariant.

To determine the appropriate transformation, we shall forsake mathematical rigour in favour of certain simplifying assumptions which happen, nevertheless, to be correct. Firstiy, considerations of symmetry and relativity will require for the restricted case the mappings:

$$
\begin{aligned}
& \mathrm{y} \rightarrow \overline{\mathrm{y}}=\mathrm{y} \\
& \mathrm{z} \rightarrow \overline{\mathrm{z}}=\mathrm{z}
\end{aligned}
$$

In the second place, considerations of relativity require that the uniformity of expansion of the wave front in one inertial frame must entail the uniformity of its expansion in every inertial frame. The alternative would 
be to abandon the concept of inertial frame. This implies the valuable clue that the transformation be linear in $x$ and $t$. This, in turn, entails that the dimensional homogeneity of the transformation equations can be preserved only if the relative acceleration between the two frames vanishes. Combining these considerations with (5.4), it follows that the required transformation must be of the form:

$$
\begin{aligned}
& x \rightarrow \bar{x}=f(x)-g(v t) \\
& y \rightarrow \bar{y}=y \\
& z \rightarrow \bar{z}=z \\
& t \rightarrow \bar{t}=f(t)+h(x / v)
\end{aligned}
$$

where $f, g, j, h$ are constant functions of their arguments. Performing the mappings $(5.5)$ on (5.1) yields:

$$
\begin{aligned}
& f^{2}(x)-2 g(v t) f(x)+g^{2}(v t)+y^{2}+z^{2} \\
= & c^{2} j^{2}(t)+2 c^{2} j(t) h(x / v)+c^{2} h^{2}(x / v)
\end{aligned}
$$

We have now simply to determine $f, 5, j, h$ such that (5.6) reduces identically to the form of (5.1). Immediate inspection suggests:

$$
f(x)=x ; j(t)=t ; g(v t)=\nabla t
$$

Substituting equations (5.7) into (5.6) yields:

$$
\begin{aligned}
& x^{2}-2 x v t+v^{2} t^{2}+y^{2}+z^{2} \\
= & c^{2} t^{2}+2 c^{2} \operatorname{th}(x / v)+c^{2} h^{2}(x / v)
\end{aligned}
$$

If we now set $h(x / v)=-v x / c^{2},(5.8)$ reduces to:

$$
x^{2}\left(1-v^{2} / c^{2}\right)+y^{2}+z^{2}=c^{2} t^{2}\left(1-v^{2} / c^{2}\right)
$$

We now find that $x$ and $t$ differ from their required values 
by a factor which is constant for any given mapping. Accordingly, we may divide by the constant factor $\left(1-v^{2} / c^{2}\right)^{\frac{1}{2}}$ to achieve the desired result. The mappings which transform uniform wave front equations into uniform wave front equations must, therefore, have the form:

$$
\begin{aligned}
& x \rightarrow \bar{x}=(x-v t) /\left(1-v^{2} / c^{2}\right)^{\frac{1}{2}} \\
& y \rightarrow \bar{y}=y \\
& z \rightarrow \bar{z}=z \\
& t \rightarrow \bar{t}=\left(t-v x / c^{2}\right) /\left(1-v^{2} / c^{2}\right)^{\frac{1}{2}}
\end{aligned}
$$

(5.10) constitute the restricted, homogeneous Lorentz transformation.

We immediately note that the preceding derivation of the Lorentz transformation did not make use of the standard heuristic devices, namely that they reduce to the Galilean transformation when $v / c \rightarrow 0$ or, in particular, that an inertial frame of special Relativity Kinematics have precisely the same significance as an inertial frame of Newtonian Mechanics. Indeed, it did emerge that the required transformation be linear, but this followed not from an analogy with Newtonian Mechanics but from the principle of relativity coupled with the formal structure of the wave front equation.

So far, as our conventional use of lower case letters indicated, we have been concerned with the establishment of a metatheorem, namely that 'the wave front equation is Lorentz covariant. Indeed, we began 
by referring to moving frames of reference, but had we regarded the situation from the four-dimensional or space-time standpoint, with $t$ as the fourth coordinate, we could have conducted the discussion solely in terms of coordinate transformations. We now turn to the level of the object theory where $x^{i}, t$ will refer to the physical coordinates of events. The results turn out to be rather interesting.

Recently a book was published on special Relativity which dealt in very large part with the twin paradox and the other so-called paradoxes of special Relativity (Prokhovnik, 1967). The author did not appear to reach any definitive resolution of the problems which he discussed. In fact, this topic would require at least one doctoral dissertation to itself. In what follows, however, we shall deal with one aspect of these problems and, we hope, resolve it to the reader's satisfaction on the basis of our general theme regarding the distinction between coordinate and frame transformations or covariance and relativity principles. We refer to the spherical wave front paradox. But first, we must prepare the ground with some comments about the relativistic phenomenon of time dilatation.

Firstly, we note that the restricted Lorentz transformations are the realization of a group, a subgroup of the complete Lorentz group, which entalls that every 
element has an inverse, which, in this case, is obtained simply by the interchange of barred and unbarred variables and the replacement of $+v$ by $-v$ in $a l l$ of its occurrences. When we deal with the object theory of Special Relativity Kinematics, the implication of the relationship between the transformations and their inverses carries an obvious but counterintuitive message. In fact, it is largely in virtue of its counterintuitive character that special Relativity aroused the interest of certain philosophers from the time of its inception, and led to so many counterrational interpretations.

In the realm of the object theory, the Lorentz transformation equations relate the physical coordinates of the wave front in one frame of reference to the physical coordinates of the same wave front in a second or 'moving' frame of reference, assuming both frames to be inertial. However, the latter is not quite so clear as many physicists who specialize in other areas are inclined to assume. In particular, there is a certain ambiguity in the expression 'same wave front'.

We have already intimated that a significant part of the philosophical literature on special Relativity has been based on misconceptions. Such philosophical misconceptions frequently aid and abet the scientific popularists, the modern day sophists, in endowing the theory with as great an air of paradox as possible in order 
to make it 'good reading'. Many of these paradoxes depend on the aforementioned nature of the relationship between a Lorentz mapping and its inverse. We now tiarn, as promissed, to a preliminary discussion of time dilatation and the so-called clock paradox, which is, incidentally, not to be confused with the twin paradox, the latter opening a veritable pandora's Box of problems. We are given two inertial frames, $K$ and $\bar{K}$, each having a clock, $C$ and $\bar{C}$. With respect to $K, \bar{C}$ is running slow in comparison with $C$. With respect to $\bar{Y}, C$ is running slow in comparison with $\bar{c}$. But that is to say that each clock is running slow with respect to the other, which is logically impossible. A facile philosophical tactic is to point out that, in fact, there is no formal contradiction since each claim pertains to a different frame of reference. So far so good. Sut then it is argued that this indicates that time dilatation is not 'real' but merely the outcome of a coordinate transformation. This is to trivialize the phenomenon by making it appear to be of no greater significance than, for example, a change of scale. It is also to make nonsense of the abiundance of experimental evidence concerning the duration of high speed particles.

Let us now indulge in one of the thoughtexperiments' of theoretical physics. Consider an inertial frame in the form of a rigid platform on which is mounted 
a clock and a light source at one end and a mirror at the other. We assume the round trip constancy of the velocity of light. Let the distance between the light source and the mirror be L. A light signal is sent out and reflected back to its source. The clock will measure the time interval between the two events as:

$$
t_{2}-t_{1}=\Delta t=2 \mathrm{~L} / \mathrm{c}
$$

We call this the proper time interval between the events. By this is meant, in this simple context, the time interval between two events at the same location. We now consider the 'same physical process' referred to a different reference frame which is moving uniformly with velocity $\vec{V}$ in the transverse direction. The length of the time interval between the beginning and end of the round trip passage of the light ray is $E$. The distance travelled by the light ray is $2\left[\mathrm{I}^{2}+(\mathrm{VE} / 2)^{2}\right]^{\frac{1}{2}}$. But, ex hypothesi, the velocity of light is constant. Hence:

$$
\begin{aligned}
& c \Delta \bar{t}=2\left[I^{2}+(V \Delta \bar{t} / 2)^{2}\right]^{\frac{1}{2}} \\
& \Delta \bar{t}=(2 L / C)\left(1-v^{2} / c^{2}\right)^{-\frac{1}{2}}=\Delta t\left(1-v^{2} / c^{2}\right)^{-\frac{1}{2}}
\end{aligned}
$$

In words, the proper time interval between two events is less than any improper time interval between them. It may appear that this result is incompatible with the principle of relativity, since it distinguishes a unique frame of reference, namely that in which a time interval is a minimum. We shall shortly dispell this. What we have just presented is the case of a 
single physical process referred to two distinct frames of reference. We now consider a different state of affairs. A similar apparatus is attached to the second or 'moving' frame, and the experiment is repeated. It will now turn out that the time interval is less in the second frame than in the first. As the twentieth century sophists would say, clocks in the second frame run both slower and faster than clocks in the first frame. This does preserve the symmetry between the two frames as required by the principle of relativity but at the apparent cost of logical consistency. In fact, the difficulty is easily resolved.

A proper frame is distinguished for a particular pair of events. In that irame, the time interval between such events will be a minimum. However, the principle of relativity is not violated, since no particular pair of events is uniquely determined or preferred. Secondly, there is no logical contradiction. A given clock which measures a proper time interval appears to 'run slow' with respect to another frame. But in the second frame a clock measures a proper time interval and appears to 'run slow' with respect to the first frame. But different pairs of events are involved in the two cases. The confusion would doubtless be alleviated if it were kept in mind that clocks do not measure the flow of a temporal substance but always and only the intervals 
between events. In this sense, time is relational rather than absolute. of course, the same could have been said Vis $\underline{\text { a }}$ is the universal time of Newtonian Mechanics. However, the fiction of absolute time could be consistently maintained therein simply because there is no quantitative difference between a proper and an improper time interval in Newtonian Mechanics.

We are now in a position to resolve the paradox of the light spheres. What we have just related in regard to the clock paradox will be common coin to most physicists who have had even passing acquaintance with Special Relativity. We thank such readers for their forbearance. What we are about to show is possibly more original and, we hope, of greater interest to the scientific reader. Consider two distinct frames of reference whose origins, 0 and $\overline{0}$, coincide at the moment that a spherical light pulse is radiated from the point of coincidence. At any later time, in accordance with the principle of relaiivity, the light wave will be a uniformly expanding sphere centred at 0 but also a uniformly expanding sphere centred at $\overline{0} \neq 0$. This, in brief, is the paradox of the uniformly expanding wave front. At the outset, let us remark that we deplore the atifude of those teachers who tell their students that this is simply an unanalyzable fact of nature, like gravitational attraction, which simply cannot be questioned. 
This is to lower science to the level of dogmatic theology. We shall make use of a greatly simplified argument, since the generalization is obviuus. We consider the case in which not only do the origins of the two frames coincide at the time of emission of the light pulse but also in which the frames are so coordinatized that the motion of $\overline{\mathrm{K}}$ is restricted to the $+\mathrm{x}$-direction with respect to K. A necessary kinematical condition for a wave front to be expanding radially from a point $O$ is that for every point on the wave front with the position vector $\vec{r}\left(t_{1}\right)$, there is a corresponding point on the same wave front whose position vector is $-\vec{r}\left(t_{1}\right)$. We shall restrict our analysis, however, to those points which lie on the $x$-axis. In what follows, we employ the term event to signify a point on a wave front at a fixed time. Accordingly, we may think of a wave front as an event surface.

In the 'stationary' frame of reference $k$, $a$ light pulse is emitted from the origin at $t=t_{0}$. At a later time $t$, an event $E_{1}$ occurs with coordinates $(X, t)$ and its simultaneous mate $E_{2}$ with coordinates $(-x, t)$. The physical coordinates of $E_{1}$ and $E_{2}$ satisfy the equation, $x^{2}=c^{2} t^{2}$. At $t=\bar{t}=t_{0}$, the origin ö of the 'moving' frame coincides with 0 . Therefore, the same light pulse must expand radially from $\bar{o}$ in the 'moving' frame. The event $E_{1}$ is recorded in the moving frame, where it has the 
coordinates $(\bar{X}, \bar{t})$. Since $\bar{K}$ is moving in the +x-direction, it is obvious that $\bar{x}<x, \bar{t}<t$. We may refer to the event in $\vec{k}$ as $\bar{E}_{1}$, but it is clear that the barred coordinates are nothing but a different label for the same event. In the language of space-time relativity kinematics, $E_{1}$ and $\bar{E}_{1}$ are simply two representations of the numerically identical point on the world line of a light ray. The relationship is given by:

$$
\left.\begin{array}{l}
x \rightarrow \bar{x}=(x-v t) /\left(1-v^{2} / c^{2}\right)^{\frac{1}{2}} \\
t \rightarrow \bar{t}=\left(t-v x / c^{2}\right) /\left(1-v^{2} / c^{2}\right)^{\frac{1}{2}}
\end{array}\right\}
$$

Just as $E_{1}(X, t)$ has the simultaneous mate $E_{2}(-X, t)$, so to $\bar{E}_{1}(\bar{X}, \bar{t})=E_{1}(X, t)$ there must correspond the simultaneous event $\bar{E}_{2}(-\bar{X}, \bar{t})$. The physical coordinates of $\bar{E}_{2}$ are given in $K$ by:

$$
\left.\begin{array}{l}
-x \rightarrow-\bar{x}=(-x+\vec{V} t) /\left(1-v^{2} / c^{2}\right)^{\frac{1}{2}} \\
t \rightarrow \bar{t}=\left(t-\vec{v} x / c^{2}\right) /\left(1-v^{2} / c^{2}\right)^{\frac{1}{2}}
\end{array}\right\}
$$

It is obvious by inspection that the coordinates

of $\overline{\bar{E}}_{1}$ given by $(5.13)$ and those of $\overline{\mathrm{E}}_{2}$ given by (5.14) both satisfy the wave front equation, $\bar{x}^{2}=c^{2} \bar{t}^{2}$. However, whereas $E_{1}$ and $\bar{E}_{1}$ were deliberately chosen to be the self-identical event, $E_{2}$ and $\bar{E}_{\hat{L}}$ are distinct events. The event $\bar{E}_{2}^{*}$ which is identical to $E_{2}$ in $\overline{\mathrm{K}}$ has the coordinates $(-\bar{x}, \bar{t})$ given by:

$$
\left.\begin{array}{l}
-x \rightarrow-\bar{x}=(-x-v t) /\left(1-v^{2} / c^{2}\right)^{\frac{1}{2}} \\
t \rightarrow \bar{t}=(t+v x / c) /\left(1-v^{2} / c^{2}\right)^{\frac{1}{2}}
\end{array}\right\}
$$

Comparing equations $(5.15)$ with $(5.14)$, we 
conclude that $\vec{E}_{2}^{*}$ is both farther from the origin and later than $\bar{E}_{2}$. (The reader will note that we have used inverse transformations whenever necessary to give all coordinate values in terms of $K$ and $S$ for purposes of comparison.) We are now able to resolve the paradox of the light spheres, not for its own intrinsic interest, but to deepen our understanding of the Lorentz transformation. The effect of the Lorentz transformation is to 'carry' light spheres into light spheres. In other words, the wave front is a symmetry or invariant of the Lorentz group. However, we are able to have a spherically symmetrical wave front in the two frames of reference, $\mathrm{K}$ and $\overline{\mathrm{K}}$, only because the transformation 'selects' different sets of events to constitute the event surfaces of the light pulse.

It is at this juncture that we find ourself in disagreement with Bunge who speaks of the Lorentz transformation as "just the relation between two different representations of events."(1967a, p. 189) In one sense he is correct, insofar as the Lorentz covariant character of the wave front equation can be determined by pure computation. However, he is wrong in supposing that the transformation is merely a formal device for representing the same physical process in different frames of reference. Moreover, since the frames of reference in question are inertial frames of special Relativity Kinematics, i.e. not 
affecting the laws of that theory, Bunge's view virtually entails that such a frame transformation is essentially the same as a coordinate transformation, but with time dependent coefficients. In fairness, we are obliged to reiterate that it depends on the meaning that one attaches to 'same physical process'. If this means the history of a certain light pulse, then Bunge's claim is essentially correct. However, a deeper view of the Lorentz transformation is that it results in representations of distinct physical processes, e.g. event surfaces, taking place in distinct frames of reference but in accordance with the same physical laws of optics as required by the principle of relativity. Since the transformation singles out different sets of events instead of representing the same events in different frames of reference, it can hardly be regarded as purely formal. Admittedly, the situation is extremely subtle as attested to by the necessity for our constant switching from lower case to upper case variables. The subtlety of this particular case arises from the fact that the specific covariance group of special Relativity Kinematics which we have studiea, namely the restricted, homogeneous Lorentz group also happens to be a relativity group of that theory. While the former is a calculable metatheorem, the latter is a testable physical hypothesis whose falsification would require a modification of the laws of optics. 
In concluding this part of our treatment of Special Relativity Kinematics, we should remark that the foregoing serves as the best possible illustration of the distinction between a pure coordinate transformation which simply applies new labels to the same physical events, and a frame transformation which, in this case, leads to the selection of different physical events in different frames of reference, but obeying the same fundamental laws. We believe that the apparent 'queerness' of the Lorentz transformation is fully accounted for by the foregoing considerations. Finally, we think it relevant to reiterate that even in the highly delimited context of special Relativity Kinematics, there is a sharp distinction to be drawn between the metatheory and the object theory.

So far, we have restricted our treatment of the Lorentz transformation to the simple case in which the origins of two coordinate systems colncide at an arbitrary initial time, the spatial axes are parallel, and the motion of the barred frame is restricted to the $+x$-direction. The resulting transformation equations (5.10) constitute the restricter, homogeneous Lorentz group of transformations. We now proceed to make certain generalizations.

Firstly, in place of the wave front equation, we consider the more general expression: 


$$
s^{2}=x^{2}+y^{2}+z^{2}-c^{2} t^{2}
$$

We define this quantity as the space-time distance, which can be shown to be the principal invariant of the homogeneous Lorentz group. In other words, a homogeneous Lorentz mapping preserves the equality:

$$
s^{2}=s^{2}
$$

Secondly, we consider the case in which the coordinatization of the two inertial frames is such that their relative velocity has three non-vanishing components. Since the latter may be reduced to the restricted case by an appropriate spatial rotation, under which $s^{2}$ remains invariant, it is obvious that the general, homogeneous Lorentz transformation is the realization of a group. However, contrary to intuition, the latter group may not be decomposed into the direct product, $R_{3} \times L_{s}$. We shall shortly explain this peculiarity of the general Lorentz transformation, although its full significance will only appear in Chapter VII, in which we formalize the Principle of special Relativity. Finally, we consider the case in which the origins of the coordinate systems do not coincide. The quantity $s^{2}$ is no longer invariant in this, the inhomogeneous case. We may settle this matter easily by rewriting the principal invariant in differential form:

$$
(d s)^{2}=(d x)^{2}+(d y)^{2}+(d z)^{2}-c^{2}(d t)^{2}
$$

which is invariant under a general, inhomogenous Lorentz mapping. It is known as the space-time interval. However, 
from the standpoint of the relativity group, (5.18) which is the differential form of $(5.16)$ is irrelevant, since the inhomogeneous term is independent of time and therefore eliminable by a pure coordinate transformation.

We remarked in Chapter II, Sec. 1 that the group property of a set of permissible mappings is by no means trivial. In the context of Classical Relativity and Special Relativity, it is possible to restrict the models of reference frames to Cartesian coordinate systems, in which case the coordinate transformations are such that their Jacobian is non-vanishing throughout all space, and it is not necessary to restrict the application to a finite region of the $V_{n}$. Hence, it is obvious that for any mapping there is an inverse, and for any succession of mappings there is a single mapping which will transform a given quantity into the original quantity.

In the case of mappings which model frame transformations, there are obvious epistemological reasons for the relativity mappings, whatever they happen to be, to possess the group property. Very simply, since, ex hypothesi, a relativity principle applies, it must be the case that if a relativistic process is referred successively to several distinct inertial frames and ultimately to the original frame, no change should be induced 'along the way' in the fundamental law of interest. To speak in terms of subjective observations, which is 
justifiable in an epistemological context, an objective process cannot be affected by the viewpoint of the observer. An objective process is characterized in relativity physics, including Newtonian, by the fact that whatever point of view, i.e. frame of reference, the observer happens to adopt, it is always possible to change that point of view back to the original one without inducing a change in the nature of the observed process. Thus, the projection of a cube on a two-dimensional surface may be a square for a particular observer. The projection will undergo a series of changes as the observer moves with respect to the cube; but whatever the situation of observer and cube at a given time, there is always possible, in principle, a single movement of the observer which will restore his original orientation with respect to the cube and such that the projection of the latter on the same two-dimensional surface will once more be a square. This reveals the objectivity of the projective properties of the cube. Clearly there is, in general, an intimate link between the objective reality of a physical pattern and its appropriate relativity group. Accordingly, since the group property of physical frame transformations is non-trivial, we should not expect to find that any set of frame transformations which happens to be mathematically representable is the realization of a group. 
The foregoing remarks apply to an interesting property of the Lorentz group which distinguishes it from the Galilean group. We have noted that the restricted Lorentz mappings are the realization of a group which is a subgroup of the complete Lorentz group. Similarly, the inhomogeneous spatial rotation group is a subgroup of the Lorentz group. However, in Newtonian Mechanics, the general Galilean transformation without rotation is the realization of a group, whereas its counterpart, the general Lorentz transformation without rotation is not. That is to say, the combination of two successive general Lorentz transformations without rotation is not necessarily equivalent to a single general Lorentz transformation without rotation. This is probably why the majority of textbooks limit their exposition to the restricted Lorentz group. The proof is rather lengthy, although not advanced, but since it is part of standard mathematics we leave it to the interested reader to try it out for himself.

In physical terms, suppose that a particle has a velocity $\vec{V}$ in an inertial frame $K$. Under a Lorentz transformation without rotation, we calculate that its image in $\overline{\mathrm{K}}$ is given by $\mathrm{k}_{1} \overrightarrow{\mathrm{V}}\left(\mathrm{k}_{1}=\right.$ const. $)$. We then make a similar Lorentz transformation to a third frame but find that the mapping from $K$ to $\overline{\bar{K}}$ does not induce the mapping, $\vec{V} \rightarrow k_{2} \vec{V}\left(k_{2}=\right.$ const. $)$. That is to say, $\overrightarrow{\bar{V}}$ will not 
generally be parallel to $\vec{V}$ but will have undergone a rotation. In general, a vector in $\mathrm{K}$ will only be parallel to its image in $\bar{B}$ when the velocity vector between $K$ and $\bar{K}$ is parallel to the velocity vector between $\overline{\mathrm{K}}$ and $\overline{\bar{K}}$. This case, however, is always reducible to the restricted Lorentz transformation by the appropriate choice of Cartesian coordinate system. The rotation of the image vector is known as the Thomas precessioil (Thomas, 1927). In short, the counterparts of what are sometimes called velocity mappings in Newtonian Mechanics are not the realization of a group in special Relativity physics.= That, of course, is not to say that velocity mappings cannot be performed in special Relativity physics. There is a well-known set of formulae for the composition of velocities in special Relativity, which are easily obtained by differentiation of the general Lorentz transformation and a little algebra. It is merely that the composition of velocities in special Relativity not only obeys a different law from the classical one but also has a different significance. As we have already remarked, we shall have occasion to refer to this matter once more in a later chapter.

We mention the Thomas precession not for the sake of completeness in our treatment of Special Relativity Kinematics, for which we are obviously not endeavouring, but to bring out the difference, alluded to sisewhere, 
between an inertial frame of Newtonian Mechanics and an inertial frame of Special Relativity Kinematics. Several writers including Einstein and Fock identify them. Einstein frequentiy alluded to Galilean frames when referring to the inertial frames of special Relativity. In fact, the most that can be said is that the total set of Galilean frames $\left\{\mathrm{K}_{\mathrm{g}}\right\}$ is coextensive with the total set of Lorentz frames $\left\{\mathrm{K}_{l}\right\}$. This will be the case if there is at least one frame which is rigorously inertial for both electrodynamics and Newtonian Mechanics. This would not, however, be a useful subject of investigation for the experimental physicists. Such an investigation would be pointless since it would presuppose that an accuracy of measurement of the second order in $\mathrm{V} / \mathrm{C}$ could be attained not only in electrodynamical experiments but also in purely mechanical ones. In the second place, however, Newtonian Mechanics is regarded only as an approximation. Consequently, a sufficiently accurate experiment would be influenced by the effects of special Relativity Mechanics. However, even if one allowed that there exist in the universe a frame which is both Galilean, in the sense of Newtonian physics, and inertial, in the sense of Special Relativity, so that in virtue of the linearity of both Galilean and Lorentz mappings, we could assert the equality $\left\{K_{g}\right\}=\left\{K_{l}\right\}$, there would still be no element to element equivalence between them. A very simple 
argument which does not depend on the Thomas precession may serve to illustrate this.

We choose an example in which all velocity vectors are parallel so that the Thomas precession cannot enter. Let us suppose that we have a 'stationary' laboratory frame $K$ with respect to which a second frame $\bar{K}$ is moving in the $+x-d i r e c t i o n$ with velocity $\vec{U}$. A particle has the velocity $\overrightarrow{\vec{V}}$ with respect to $\vec{K}$. According to Newtonian Kinematics, the magnitude of the velocity of the particle with respect to $K$ must be $|\vec{U}+\vec{V}|=|\vec{U}|+|\overrightarrow{\vec{V}}|$. According to special Relativity Kinematics, the particle will have the velocity $(\vec{U}+\overrightarrow{\bar{V}}) /\left(1+U \bar{V} / c^{2}\right)$ with respect to K. Bridgman(1962) expressed serious doubts concerning the relativistic law for the composition of velocities on the ground that it involves two distinct differential operators $d / d t$ and $d / d \bar{t}$. However, what follows would depend only on direct observation. Specifically, we introduce a third frame $\overline{\bar{K}}$ which is the rest frame of the particle. Whether or not a particle is at rest in a particular frame is, of course, determined by direct observation. By simple logic, the velocity of the rest frame with respect to $K$ must be $(\vec{U}+\overrightarrow{\vec{V}}) /\left(1+U \bar{V} / c^{2}\right)$. However, if we use the formula of Newtonian Kinematics, the rest frame of the particle will have the velocity $\vec{U}+\overrightarrow{\vec{V}}$ with respect to $K$. In short, while the equality $\left\{\mathrm{K}_{\mathrm{g}}\right\}=\left\{\mathrm{K}_{1}\right\}$ conceivably holds, the corresponding transformation 
laws relate different elements of the set of inertial

frames. In more formal language:

$\{K\}$ is the basic set of reference frames.

g(=df. kinematically equivalent up to a Galilean

transformation) is a relation which is reflexive, symmetric and transitive in $\{K\}$.

$I(=d f$. kinematically equivalent up to a Lorentz

transformation) is a relation which is reflexive, symmetric and transitive in $\{K\}$.

$\left\{K_{g}\right\}=\mathrm{df}$. $g\{K\}$ is the equivalence class of inertial frames of Newtonian Mechanics.

$\left\{K_{I}\right\}=d f . I\{K\}$ is the equivalence class of inertial frames of Special Relativity Kinematics.

$\left\{K_{g}\right\} \subset\{K\}$ is an equivalence class of $\{K\}$.

$\left\{\mathrm{K}_{I}\right\} \subset\{K\}$ is an equivalence class of $\{K\}$.

The elements of $\left\{\mathrm{K}_{g}\right\}$ are $a, b, c, \ldots$

The elements of $\left\{K_{I}\right\}$ are $\alpha, \beta, \gamma, \ldots$

$G$ is a binary operation on $\left\{K_{g}\right\}$.

I is a binary operation on $\left\{K_{1}\right\}$.

$=$ is a binary relation which is reflexive, symmetric and transitive in $\{K\}$.

$\left\langle\left\{K_{g}\right\}, G,-1, I\right\rangle$ is a group $\}$.

$\left\langle\left\{K_{I}\right\}, I,-1, I\right\rangle$ is a group $\mathscr{L}$.

$\theta^{\prime}=\alpha$

$b^{\prime}=\beta$

$a G b \rightarrow c ; \alpha \perp \beta \rightarrow \gamma$

$(a G b) \cdot \neq \gamma$

The foregoing merely proves that in spite of

the putative identity of the set of Galilean frames and 
the set of Lorentz frames, the structures $\boldsymbol{\xi}$ and $\mathcal{L}$ are of different types. Accordingly, one may not speak

indiscriminately of a Galilean frame to denote either an element of the first member of the structure $\boldsymbol{\xi}$ or an

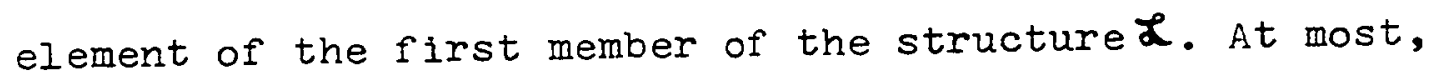
one would be entitled to speak indiscriminately of inertial reference frames, but even this is a practice which we would strongly discourage. In fine, to speak, in the manner of Einstein, of Galilean frames is to treat the relatively complex structure of a group as though it were that of a mere set.

$$
\text { If it had been the case that the relativity }
$$
group of electrodynamics were realized by non-linear transformations, the confusion between a Galilean frame and a Lorentz frame would never have arisen. In fact, however, we have already noted that frames of reference belong to the category of mechanical objects. Insofar as electrodynamics requires inertial frames, the latter must be regarded as objects which belong to the theory of electrodynamics. Thus, the foregoing discussion ceases to be of such great importance for physics, albeit of considerable methodological significance, since the mappings between mechanical inertial frames and electrodynamic inertial frames must be of the same kind. This is the rational basis for the development of the program of Special Relativity Mechanics, which we treat in the 
following chapter. This program is not based on the fixity of meaning of inertial frame, namely a frame that moves uniformly (relative to what?). It is quite conceivable that an accelerating frame could serve as an inertial frame if only the laws of nature were different. Having perused several introductory treatments of relativity physics, we are surprised that the student is not generally more confused than he actually is, since, almost without exception, there is a serious lacuna in the logical development. We quote from one of the more highly respected textbooks:

$$
\begin{aligned}
& \text { "Having obtained the Lorentz transformation } \\
& \text { to replace the incorrect Galilean } \\
& \text { transformation, we can now proceed to } \\
& \text { the second stage and require that the } \\
& \text { laws of mechanics, in common with all of } \\
& \text { physics, shall have the same form in all } \\
& \text { uniformiy moving systems." (Goldstein, 1950) } \\
& \text { But the Galilean transformation is correct! }
\end{aligned}
$$

That is to say, it is a realization of the relativity group of Newtonian Mechanics. Can the Lorentz covariance of Maxwell's laws serve to falsify the laws of Newtonian Mechanics? This is an absurdity. There is no apparent reason why different theories should not have different transformation properties. The point is that electrodynamics includes mechanical objects within its purview. Hence, Newtonian Mechanics which is inconsistent with electrodynamics must be modified. Otherwise, the theory of electrodynamics would be inconsistent. Conversely, one 
could argue that Maxwell's laws should be modified to make them Galilei covariant. In fact, both paths were pursued but only one proved fruitful. However, we shall show in Chapter VIII that from the deeper standpoint of General Relativity, the coexistence of a Galilei covariant mechanics and a Lorentz covariant electrodynamics is not only a physical but also a mathematical impossibility. 


\section{CHAPTER V \\ SPECIAL RELATIVITY MECHANICS}

At the close of the preceding chapter, we briefly indicated the rationale for the general program of Special Relativity physics. In the case of electrodynamics, this program had, in a sense, been carried out prior to the formulation of the Principle of special Relativity, insofar as the basic laws of that theory are covertly Lorentz covariant as they stand. However, Newtonian Mechanics is not a Lorentz covariant theory. Hence, in executing the program of special Relativity physics, it is natural to anticipate a modification of Newton's laws.

Fortunately, it was shown by Minkowski(1908) that a formalism could be employed in which properly constructed equations are automatically Lorentz covariant. This formalism plays a similar role vis $\underline{\mathrm{a}} \mathrm{v}$ is the Lorentz group of coordinate transformations to that played by the elementary three-vector formalism vis $\underline{\text { a }}$ vis the Galilean group of transformations. The particular version of this formalism which we shall adopt for the time being involves the replacing of the ordinary time parameter by a fourth coordinate ict $=x^{4}(i=\sqrt{-1})$. This particular version of the so-called four-vector formalism is beginning to go out of fashion. Today, many writers, 
e.g. Bunge, Fock, et al., favour the use of the real time coordinate ct. The principal objections to the original Minkowski version are that it treats of rotations through imaginary angles and, in general, involves reference to imaginary quantities, which tends to obscure the physical content of theoretical laws. Furthermore, it is of no value in the Theory of General Relativity. The principal advantages of the imaginary coordinate are that it enables one to treat the space-time manifold as though it were formally Euclidean, which leads to some notational simplification, and, moreover allows one to continue to ignore the distinction between the covariant and contravariant components of a vector. On balance, we have decided that the imaginary coordinate is well suited to our purposes. There is no danger in adopting the most convenient formalism for a particular purpose, provided only that one does not confuse the special properties of the formalism with those of physical reality.

The purpose of this chapter is quite straightforward, namely to provide supporting evidence for the thesis that the Principle of Special Relativity has physical content, i.e. that, as distinct from Lorentz covariance, per se, it belongs to the object language of physics. Our general procedure will be the rather workaday one of taking Newtonian laws and, with the aid of the four-vector formalism, of rewriting them in 
manifestly Lorentz covariant form. The point will be to show that the laws which are obtained in this way differ in physical content from those of Newtonian Mechanics. For the benefit of the reader who is unfamiliar with the four-vector formalism, we shall begin with a very brief sketch of the manner of its working. We employ a four-dimensional orthogonal coordinate system with the $x^{i}$ having their usual significance and with the fourth coordinate $\mathrm{x}^{4}=$ ict. It is then clear that the space-time interval: $(d s)^{2}=(d x)^{2}+(d y)^{2}+(d z)^{2}-c^{2}(d t)^{2}$ may be represented by the homogeneous quadratic form:

$$
(d s)^{2}=\delta_{\mu v d x^{\mu} d x^{v} \quad(\mu, v=1,2,3,4)}
$$

since our purpose is to construct geometrical objects which are analogous to three-vectors, we require that the linear, homogeneous transformation:

$$
x^{\mu} \rightarrow \bar{x}^{\mu}=\alpha_{\mu \nu} x^{\nu}
$$

be such that the space-time interval be an invariant. That is to say, under the transformation (6.2), we must have:

$$
\left(d x^{\mu}\right)^{2}=\left(d \bar{x}^{\mu}\right)^{2}
$$

Employing the transformation $(6.2)$ in $(6.3)$, we have:

$$
\alpha_{\beta \gamma} d x^{\gamma} \alpha_{\beta \delta} \delta x^{\delta}=\delta_{\mu r} d x^{\mu} d x^{\nu}
$$

or, $\quad \alpha_{\beta \gamma} \alpha_{\beta \delta} d x^{\gamma} d x^{\sigma}=\delta_{\mu v d x^{\mu} d x^{v}}$

clearly, (6.5) can only be satisfied identically when:

$$
\alpha_{\beta \gamma} \alpha_{\beta} \delta=\delta_{\gamma \delta}
$$

The superficial appearance of $(6.6)$ is that of 
one of the orthogonality conditions under a rotation in three dimensions; the only difference is that the indices run from 1 to 4 instead of from 1 to 3. Thus, we may formally treat a homogeneous Lorentz transformation as a rotation of a four-dimensional Cartesian coordinate system. However, it should be abundantly clear to the reader that we are in the mathematical universe of discourse. To forget this would be profoundly misleading. The Minkowski coordinates are useful precisely in virtue of their formal analogy with Cartesian coordinates. However, we must not forget the imaginary character of $x^{4}$. If we were using real coordinates, (6.1) could not be expressed in its present form. In particular, if real coordinates were employed, the metric quadratic form could not possibly be transformed to positive definite form by any real transformation. This is but one reason why Minkowski coordinates would be nothing but a handicap in the more complex arena of General Relativity. But at that point, in any case, we need a Riemannian as distinct from a Euclidean or pseudo-Euclidean metric.

We now define a four-vector in Minkowski space as any quantity that transforms like the $\mathrm{d} \mathrm{x}^{\mu}$. It follows from this condition that the square of the magnitude of a four-vector must be a Lorentz invariant. It is called the norm of the four-vector. In spite of the formal analogy with Euclidean geometry, differences appear as 
soon as we consider the invariant norm. As a consequence of the imaginary character of $x^{4}$, it is possible for the norm of a four-vector to be zero even though it have non-vanishing components. In seneral, we have:

$$
(d s)^{2}=\left(d x^{\mu}\right)^{2}=\left(d x^{i}\right)^{2}-c^{2}(d t)^{2}
$$

If the norm of the four-vector is greater than zero, i.e. $\mathrm{dx}^{i}$ is the dominant quantity, we have a space-like vector. If it is less than zero, i.e. $\mathrm{dx}^{4}$ is the dominant quantity, we have a time-like vector. (Employing real coordinates, we may interchange these relations.) If it is equal to zero, we have a null-vector, which is most important in special Relativity physics, since it obviously corresponds to the condition of light propagation, i.e. $\left(d x^{i}\right)^{2}=c^{2}(d t)^{2}$. In this four-dimensional context, it is customary to speak of the light-cone as defined by $(d s)^{2}=0$. This concept is intuitively useful in the following way. Suppose that we have two neighbouring events: $E_{1}\left(X^{\mu}\right)$ and $E_{2}\left(X^{\mu}+d X^{\mu}\right)$. The light-cone with $E_{1}$ as centre is given by $(d s)^{2}=0$. Now, if $E_{2}$ lies on the light-cone, $E_{1}$ and $E_{2}$ may be connected by a light signal. If $E_{2}$ is inside the region defined by the light-cone, then $\mathrm{dX}^{\mu}$ is a time-like vector. If $E_{2}$ lies outside of this region, then $\mathrm{dx}^{\mu}$ is space-like. Furthermore, it is easy to show that if a vector is time-like, there is always a permissible trarsformation which makes the spatial 'part' of the vector vanish; whereas, if a vector is space-like, 
there is always a permissible transformation which makes the temporal 'part' of the vector vanish. Intuitively, if the norm of the space-time distance is non-positive, the two events may be regarded as simultaneous, whereas if the norm of the space-time separation is greater than zero, the two events are absolutely successive.

We now define the time-track on our mathematical manifold by the definite linear functions:

$$
\xi^{i}=x^{i}\left(x^{4}\right)
$$

Next, we choose a coordinate system such that the curve $\xi^{i}$ lies along the $\mathrm{x}^{4}$-axis. With respect to such a coordinate system, we have:

$$
\mathrm{x}^{1}=0
$$

for the spatial coordinates of the time-track. Clearly, the values of $x^{4}$ correspond to the path length $s$ and also to the proper time of which we spoke in the preceding chapter. By a suitable choice of scale, we make $d x^{4}=d s$. It is obvious that the displacement ds is a time-like vector with the components $(0,0,0$, icdt). Let us replace the parameter $x^{4}$ of $(6.8)$ by the space-time distance $s$. Then we represent the curve by:

$$
\xi^{M}=x^{M}(s)=x^{M}(i c t)
$$

We define the real auxiliary variable $\tau$ by:

$$
d \tau=\frac{d s}{i c}
$$

From (6.7), (6.9) and (6.11) we deduce the equality:

$$
d \tau=d t
$$


We have just demonstrated that it is always possible to choose a coordinate system such that the real coordinate time $t$ corresponds to the proper time $\tau$. Furthermore, we have shown that in such a system the proper time interval must be orthogonal to the spatial axes. In general, however, the spatial components of the displacement ds will be non-vanishing. We now determine the relationship between $d \tau$ and at for the general case. Inserting (6.11) in (6.7), we have:

$$
-c^{2}(d \tau)^{2}=\left(d x^{i}\right)^{2}-c^{2}(d t)^{2},
$$

from which there immediately follows:

$$
d \tau=\left(1-v^{2} / c^{2}\right)^{\frac{1}{2}} d t
$$

which is Lorentz invariant.

We now have the necessary tools at our disposal to deal with Special Relativity Mechanics. We wish to underline the important point that all of the preceding discussion, although aimed at a physical application, strictly belongs to the realm of mathematics. We have lapsed into the language of physics with such terms as 'event', 'time-track', 'Iight propagation', etc., only as an aid to intuition. None of these terms were essential to the mathematical development. In short, nothing so far said has had anything to do with the physical world. We have simply constructed a formalism which may be so interpreted as to lend itself to the expression of physical laws. The laws of that formalism belong to the mathematical 
framework of physics. We now turn to the object theory of Special Relativity liechanics. We stress, once again, that our treatment of physical principles is not intended to be exhaustive but merely, we hope, instructive. In particular, to keep matters fairly simple, we Iimit our discussion to point mechanics.

As mentioned at the beginning of the chapter, we shall employ the simple (and probably simple-minded) stratagem of modifying Newton's equations in such a way that the resulting equations are Lorentz covariant. Naturaliy, we do not imply by this procedure that a Lorentz covariant formula must, a fortiori, correspond to a physically true law of nature. This would be just as unfounded as to argue that a Galilei covariant formula is necessarily the expression of a true law of nature. We do, however, intend to show that such a procedure leads to testable physical consequences. For information on the actual confirmation of such consequences, the reader should turn to any standard textbook on the subject.

It is natural to begin our account with the concept of the velocity of a point particle. The classical velocity $\mathrm{dx}^{i} / \mathrm{dt}$ is not a vector in four-space. Obviously, since time is not universal in special Relativity physics, it would be difficult to construct a four-vector with its aid. We turn, therefore, to the Lorentz invariant proper time $\boldsymbol{\tau}$. We interpret $\boldsymbol{\tau}$ very crudely and strictly 
as an aid to intuition in operationalistic terms as the time measured by a clock which is attached to the moving particle. A change of reference frame obviously cannot alter the value of $\tau$, since it does not involve the transmission of a signal but depends on the direct coincidence of events. We may express the four-velocity $U^{\mu}$ by :

$$
\frac{d x^{n}}{d \tau}=\frac{d X^{M}}{d t} \frac{d t}{d \tau}
$$

By (6.14), we may express (6.15) as:

$$
U^{\mu}=\frac{d x^{\mu}}{d \tau}=\left(1-v^{2} / c^{2}\right)^{-\frac{1}{2}}\left(\frac{d x^{i}}{d t}, i c\right)
$$

The norm of $U^{\mu}$ is easily obtained:

$$
(U)^{2}=\delta_{\mu v} U^{M} U^{v}=\left(1-v^{2} / c^{2}\right)^{-1}\left(v^{2}-c^{2}\right)=-c^{2} \text { (6.17) }
$$

In short, we reach the rather interesting result that the norm of the four-velocity is a universal invariant. Moreover, we note for future reference that $U^{M}$ is a time-like vector. The universal invariance of the norm of $U^{\mu}$ should not be regarded as too surprising, since differentiation was with respect to $\tau$ which is invariant, and the frame of reference is the rest frame of the particle in which time has been coordinatized by ict. Intuitively, the measurement of the four-velocity of the particle is relative to the velocity of light, which must be the same for all inertial frames. (Essentially the same result would have been obtained using the real coordinate ct.)

Instead of working out the components of the 
four-acceleration of a particle, which involves some tedious mathematics, we shall follow the time-honoured and easier path of deriving a Lorentz covariant formulation of the conservation of momentum for the collision of two particles. This will, in turn, lead us to the definition of the four-force, of ten called the Minkowski force. Incidentally, this type of example is one of the $f e w$ physical cases in point mechanics for which a Lorentz covariant counterpart is possible, since the nature of special Relativity rules out instantaneous action at a distance. Special Relativity fully comes into its own only in the case of field theories. The conservation of momentum in elementary point mechanics is given by the well-known formula:

$$
m_{1} \vec{U}_{1}+m_{2} \vec{U}_{2}=m_{1} \vec{V}_{1}+m_{2} \vec{v}_{2}
$$

We naturally assume that the velocities must be replaced by four-velocities and the masses by some quantities which are Lorentz invariant. Consequently, we rewrite $(6.18)$ as :

$$
\mathrm{M}_{1} \mathrm{U}_{1}^{\mu}+\mathrm{M}_{2} \mathrm{U}_{2}^{\mu}=\mathrm{M}_{1} \mathrm{~V}_{1}^{\mu}+\mathrm{M}_{2} \mathrm{~V}_{2}^{\mu}
$$

More generally, we may write:

$$
M_{n} U_{n}^{\mu}=\text { const. }
$$

Inserting $(6.16)$ in $(6.20)$, we obtain:

$$
M_{n}\left(1-v^{2} / c^{2}\right)^{-\frac{1}{2}}\left(d x^{1} / d t, 1 c\right)_{n}=\text { const. }
$$

We now define the quantity:

$$
m=d f \cdot M\left(1-v^{2} / c^{2}\right)^{-\frac{1}{2}}
$$


We call $\mathrm{m}$ the relativistic mass. Clearly, in an inertial frame in which $m$ is at rest, we have $m=M$. Consequently, $M$ is an invariant which we henceforth denote by $m_{0}$ and call the rest-mass or proper mass. It has the same value in all inertial frames and may, therefore, be employed to define a Lorentz covariant momentum given by:

$$
\mathrm{p}^{\mu}=\mathrm{df} \cdot \mathrm{m}_{0} \mathrm{U}^{\mu}=\left(\operatorname{md} \mathrm{x}^{i} / \mathrm{d} t, \mathrm{imc}\right)
$$

The direct significance of the foregoing for this dissertation is that the imposition of Lorentz covariance on the law of conservation of momentum led to a physical consequence, namely that the Newtonian or classical concept of mass must be abandoned in special Relativity Mechanics. Many writers with a similar motivation to our own prefer to say that a factual consequence of Special Relativity Mechanics is that the mass increases with the velocity, contrary to the doctrine of Newtonian Mechanics. However, while this is true, it refers to the relativistic mass, which does not occur in Newtonian Mechanics. Another frequent declaration is that the classical mass is equivalent to the proper mass of special Relativity Mechanics. But since the proper mass is always measured in its rest frame, it clearly has a different meaning from that which Newton attached to the concept of mass. The proponents of the latter view are, in effect, using a 'correspondence principle', according to which Special Relativity Mechanics reduces to Newtonian Mechanics 
as $v / c \rightarrow 0$, as a constitutive law of special Relativity physics instead of as an occasional heuristic guide. We have just employed the classicle principle of the conservation of momentum as a guide to the construction of a four-vector; but the classical principle of momentum conservation certainly does not belong to the Theory of Special Relativity. In short, it appears that when we speak either of relativistic mass or of proper mass, we are guilty of semantic confusion in identifying either one with the Newtonian quantity of matter. We prefer, therefore, the more cautious statement that the fourmomentum of a particle, defined as the product of its rest-mass and its four-velocity, is conserved. We shall shortly see that it is semantically admissible to compare Newtonian and Special Relativity Mechanics at certain points, but this is not one of them. It is philosophically more profitable to regard special Relativity Mechanics as a distinct theory from Newtonian Mechanics than as a modification of it.

By the same token, writers who state that 'PM $=(\vec{p}, i m c)$ ' signifies that the spatial components of the four-momentum vector are identical with the classical momentum are guilty of a similar, if not worse, error. This error is probably bolstered by the true but physically irrelevant statement that the three spatial components of a four-vector constitute a geometrical object which 
transforms like a three-vector. In fact, of course, such a transformation simply represents a new coordinatization and has no bearing on the principle of relativity. More significantly, the spatial components $P^{i}$ of the fourmomentum vector are given in the restricted case by:

$$
\mathrm{P}^{i}=\mathrm{m}_{\mathrm{O}} \overrightarrow{\mathrm{V}}\left(1-\mathrm{V}^{2} / \mathrm{c}^{2}\right)^{-\frac{1}{2}}
$$

Clearly, (6.24) behaves quite differently under a Galilean frame transformation from the Newtonian momentum. In particular, if $(6.24)$ is subjected to a Galilean transformation under which $c$, of course, would no longer be an invariant, it would be found that the momentum relative to the second frame would not be in the same proportion to the momentum in the first frame as the velocity of the second frame relative to the first - a result which is at complete variance with Newtonian Mechanics.

In fact, we may consider two possibilities for the Newtonian physicist, when confronted by $(6.24)$. In the first case, he might know, although failing to understand why, $c$ is a universal constant in all inertial frames of reference, so that he would leave it untouched in subjecting $(6.24)$ to a Galilean transformation. Suppose a transformation is made with respect to an inertial frame moving with velocity $\vec{U}$ in the $+x-d i r e c t i o n$ with respect to the original frame. Then:

$$
\mathrm{P}^{1} \rightarrow \overrightarrow{\mathrm{P}}^{-1}=\mathrm{m}_{0}(\overrightarrow{\mathrm{V}}-\overrightarrow{\mathrm{U}})\left[1-(\mathrm{V}-\mathrm{U})^{2} / \mathrm{c}^{2}\right]-\frac{\frac{1}{2}}{2}
$$


In accordance with Newtonian Mechanics, dividing (6.25) by $(6.24)$ we should get the identity:

$$
\begin{aligned}
& m_{0}(\vec{V}-\vec{U})\left[1-(V-U)^{2} / c^{2}\right]^{-\frac{1}{2}} \cdot\left(1-v^{2} / c^{2}\right)^{\frac{1}{3}} / m_{0} V \\
= & |\vec{U}| /|\vec{V}|
\end{aligned}
$$

But, in fact, $(6.26)$ is not satisfied identically, although it naturally approaches an identity as $\vec{U} \rightarrow 0$.

In the second case, we suppose that the Newtonian physicist is unaware of the invariance of $c$ but has measured it in a stationary frame. He will then include in the Galilean transformation the mapping:

$$
\vec{c} \rightarrow \overrightarrow{\vec{c}}=\vec{c}-\vec{U}
$$

We leave it to any reader who has time to waste to verify that the insertion of $(6.27)$ in $(6.26)$ would still be inconsistent with Newtonian Mechanics.

The most we can say of the symbol on the left hand side of $(6.24)$ is that it represents the same kind of geometrical object as the momentum of classical physics. But the two are, on no account, to be identified, as is the practice in many textbooks on the subject. The writers of such books would not, of course, represent the spatial components of the four momentum by $(6.24)$ but rather by:

$$
\left\langle\mathrm{P}^{1}, \mathrm{P}^{2}, \mathrm{P}^{3}\right\rangle=\overrightarrow{\mathrm{p}}=\mathrm{m} \overrightarrow{\mathrm{V}}
$$

This has exactly the same appearance as the Newtonian definition of momentum and, moreover, the same covariance properties under a rotation of coordinates. However, the physicist carelessly overlooks the point that ' $m$ ' in (6.28) 
denotes the relativistic mass not the Newtonian mass of the particle.

So long as we are working with Newtonian Wechanics, the meaning of ' $m$ ' must be made explicit as in (6.24). In fine, we have exposed a compound confusion, namely between covariance and relativity principles and between two distinct theories. An incidental but important moral of this is that ore should not take correspondence principles too seriously. They emphatically do not belong to physical theories, and their heuristic value is explained not on a physical basis but on an epistemological one. The difference in magnitude between the spatial components of the four-momentum vector and the Newtonian momentum is very small for low velocities and vanishes in the trivial case of the rest frame of the particle. This, plus the fact that pre-Einsteinian physicists were not completely blind in the making of measurements, explains why correspondence principles seem to work. However, we have shown that the two theories of Newtonian Mechanics and Special Relativity Mechanics are quite distinct. The concepts which occur in them, while often bearing the same name and entering analogous logical relationships, denote different entities. Moreover, the difference in the two theories which we have discussed has been exposed on the basis of their satisfying different relativity groups! To indulge in a siogan, the comparison 
of (6.24) and (6.28) entitles us to assert that 'Covariance is not enough!'

We have already mentioned that Newtonian (instantaneous, action-at-a-distance) forces cannot be incorporated into special Relativity physics. In particular, there is no Lorentz covariant generalization of 'Newton III'. However, there is a natural counterpart to 'Newton II':

$$
F^{M}=\frac{d P^{M}}{d \tau}=m_{0} \frac{d U^{\mu}}{d \tau}
$$

It has already been noted (6.17) that the norm of the four-velocity is equal to a universal constant. Rewriting (6.17) as:

$$
\left(U^{\mu}\right)^{2}=-c^{2},
$$

and differentiating with respect to $\tau$, we get:

$$
U^{\mu} \cdot \frac{d U^{\mu}}{d x}=U^{\mu} \cdot F^{M}=0
$$

Let us now ascertain the components of the

four-force. From (6.23) and (6.29), we have:

$$
\begin{aligned}
& F^{M}=\frac{d}{d \tau}(\vec{P}, i m c)=\frac{d t}{d \tau} \frac{d}{d t}(\vec{P}, i m c) \\
& F^{M}=\left(1-v^{2} / c^{2}\right)^{-\frac{1}{2}}(\vec{f}, i \dot{c} c)
\end{aligned}
$$

From (6.16), (6.31) and (6.33), we have:

$$
\left(1-v^{2} / c^{2}\right)^{-1}(\vec{v}, i c) \cdot(\vec{f}, i m c)=0
$$

Hence,

$$
\vec{V} \cdot \vec{F}=c^{2} \dot{m}=0
$$

and, $\quad(i / c) \vec{V} \cdot \vec{F}=i \dot{c} c$

Inserting (6.36) in (6.33), we have:

$$
F^{\mu}=\left(1-v^{2} / c^{2}\right)^{-\frac{1}{2}}(\vec{F},(1 / c) \vec{V} \cdot \vec{F})
$$

which must be a four-vector by virtue of (6.29). 
It is apparent from (6.37) that the 'direction' of $\mathrm{F}^{\mu}$ will depend on the ordinary velocity of the particle on which it is acting. Consequently, two four-forces which are parallel with respect to an inertial frame of reference will not, in general, be parallel in a second frame which differs from the former by a Lorentz transformation. This is one reason why there is no Lorentz covariant counterpart of 'Newton III'. However, this very fact will give us a deeper understanding of the force law of electrodynamics, which we consider in the next chapter.

In addition, an important physical consequence arises. Since the norm of $\mathrm{F}^{\mu}$ is invariant, it follows that the Newtonian force which acts on a particle must vary with the velocity of the particle. It is possible to demonstrate this result by means of a simple computation which avoids the usual complexities of subjecting Newtonian forces to Lorentz transformations. The norm of $F^{\mu}$ is given by:

$$
\begin{aligned}
(F \mu)^{2} & =\left(|\vec{F}|^{2}-\frac{|\vec{V}|^{2}|\vec{F}|^{2}}{c^{2}} \cos \theta\right)\left(1-v^{2} / c^{2}\right)^{-1} \\
& =|\vec{F}|^{2} \sin ^{2} \theta=k
\end{aligned}
$$

Since $F^{\mu}$ is a space-like vector, it is permissible to transform to the rest frame of the particle in which the time component of $F^{\mu}$ vanishes. Physically, this simply amounts to the force's acting on a particle whose instantaneous velocity is zero. The norm of $\bar{F}^{m}$ is given by:

$$
\left(\bar{F}^{\mu}\right)^{2}=|\overrightarrow{\bar{F}}|^{2}=k
$$


Equating (6.38) and (6.39), we have:

$$
|\overrightarrow{\mathrm{F}}|^{2}=|\overrightarrow{\mathrm{F}}|^{2} \sin ^{2} \theta
$$

Hence, $\quad \vec{F}>\overrightarrow{\vec{F}}$

The Newtonian case would hold rigorously only in the highly restricted circumstance in which $\vec{V}$ and $\vec{F}$ are parallel so that $\cos \theta=1$. In this case, we would have:

$$
\overrightarrow{\mathrm{F}}=\overrightarrow{\overrightarrow{\mathrm{F}}} \text {, }
$$

which is consistent with Newtonian Wechanics.

$$
\text { It may be objected that (6.41), on our own }
$$

argument, has no meaning since it pertains to Newtonian forces but was deduced from the laws of special Relativity Mechanics. Are we not guilty of the very error which we have ascribed to others? While it is true that one must avoid the 'semantic mixing' of two distinct theories, it is still possible to establish 'contacts' between them. With due semantic care, it is possible to demonstrate that one theory is incompatible with another. Thus, while (6.41) is expressed in the language of Newtonian Mechanics, it is not a theorem of Newtonian Mechanics but is a theorem of special Relativity Mechanics. Moreover, it shows a physical hypothesis of Newtonian Mechanics to be false. There is nothing in this case which is comparable with the identification of the proper mass of special Relativity Mechanics with the Newtonian mass. We wish to stress that the transformation: $F^{\mu} \rightarrow \bar{F}^{\mu}$, which was employed in our computation, belongs 
to the relativity group of point mechanics and not merely to the covariance group. That is to say, it did not correspond to a rotation of spatial coordinates but to a change of reference frame, since it involved the $\mathrm{x}^{4}$-coordinate of the Minkowski system. A velocity may be transformed away by a change of reference frame but not by a new coordinatization of the same frame. In summary, we have shown that by imposing the condition that a theory of point mechanics be Lorentz relativistic rather than Galilei relativistic, we are led to definite physical consequences. It follows that the Principle of Special Relativity must belong to the object language of physics, even though its more general metatheoretical counterpart, the Principle of Lorentz Covariance, does not. 


\section{CHAPTER VII}

\section{ELECTRODYNAMICS}

The modifications of the laws of mechanics which were brought about by making those laws Lorentz covariant may strike the newcomer to relativistic physics as rather Procrustean, and the activity of writing the new laws in the four-vector formalism as a mere mathematical exercise. In the case of Classical Electrodynamics, however, the situation is quite different. No fundamental change is brought about by writing its laws in manifestly Lorentz covariant form, although, as we shall see, greater insight is thereby achieved. In fact, the Lorentz covariant formalism turns out to be more natural than the classical formalism. In its classical form, the most unsatisfactory aspect of electromagnetic theory is the distinction between the electric and magnetic fields. What appears in one frame of reference as the manifestation of a magnetic field will appear in another as an effect of the electric field. While this does not contravene the princlple of relativity, it is epistemologically unsatisfying. For example, the magnetic force acting on a particle which moves with velocity $\vec{V}$ is given by $\vec{F}=(q / c)(\vec{V} \times \vec{B})$. This doesn't even have the form of a fundamental law, since the right hand side depends on the state of motion of the frame of reference. In particular, the magnetic force 
could be 'transformed out of existence' simply by referring it to another inertial frame, which may make one wonder whether it was really there in the first place. Despite the naturalness of the four-dimensional formalism in the case of Electrodynamics, the least fruitful approach to a genuine appreciation of the Lorentz covariance of Maxwell's laws of Electrodynamics would be to write them in their classical form and then to perform the purely formal task of rewriting them in four-dimensional, i.e. manifestly Lorentz covariant, form. In this way, the physics which underlies the Lorentz covariance of Maxwell's laws would be entirely lost, especially to a philosopher. The situation is not analogous to that of Newtonian Mechanics in which the introduction of four-dimensional formalism forces a modification of the physical content of the laws of interest.

Accordingly, before making use of the fourdimensional formalism, we shall examine some simple situations which serve to bring out the relationship between the electric and magnetic fields from the standpoint of the Principle of Special Relativity. The simplicity of the basic physical situations which we intend to examine is quite justified as a basis for generalization provided that one pays more than lip-service to the concept of the field. If the field description of electromagnetic phenomena is correct, then it is possible to 
transform a field point from one frame of reference to another regardless of the simplicity or complexity of the sources of that field. In fact, as Bunge has shown(1967a, p. 164), the field concepts are logically independent of the source concepts, so that one cannot say that the one is more fundamental than the other.

Thus, we come to the apex of our discussion of the Principle of special Relativity. The theory of Electrodynamics is the true physical basis of special Relativity physics. It was poincaré who noted in 1904 that Maxwell's equations are covariant under Lorentz transformations. Shortly thereafter, Einstein published his epochal paper: on the Electrodynamics of Moving Bodies(1905) which heralded the beginning of the Theory of Special Relativity.

There are many ways in which this chapter could be presented. Throughout this dissertation we have attempted to steer a course which would be of interest both to the physicist and to the philosopher who has some knowledge of physics. We shall continue to adhere to this course in the present chapter, which is the justification for the particular mode of presentation that we now adopt. In brier, we shall begin with a presentation of the classical version of the theory which we hope will be comprehensible to the majority of phitosphical readers. However, in presenting the classical theory, we will not 
attempt to hide our knowledge of special Relativity but shall emphasize those aspects of the physics which II Iuminate the basis of the Lorentz transformation, our purpose being to show that the latter is not simply a formal device but the representation of a fundamental aspect of physical reality.

our treatment thereafter will be more formal and, for the first time, we shall utilize tensors of the second rank. This will help to prepare the philosophical reader for the eventual confrontation with General Relativity in chapter VIII. However, to take as small a step as possible at each stage, we shall continue to employ the Cartesian coordinates of Minkowski, so that no distinction will be made between the covariant and contravariant indices. While objectionable, on some grounds, it will, at least, help to clarify the distinction between a flat Euclidean or pseudo-Euclidean manifold and a Riemannian manifold in which the aforementioned distinction is inescapable. Finally, since this is not a treatise on physics, as the reader by now knows, we shall limit the treatment to the behaviour of the electromagnetic field in a vacuum(but Cf. Bunge, 1967a, pp. 160f.). That is to say, there will be little or no talk, except heuristically, of dielectrics and conductors. In our sparse universe, we shall admit electrically neutral frames of reference, the electromagnetic field, and point charges. 
The scientifically trained reader may omit or skim over the early parts of this chapter. In the Appendix to the present chapter, we shall make up for one of our omissions by endowing our point charges with mass. This, again, will prepare the ground for the chapter on General Relativity.

There are two kinds of electric charge which are nominally designated as positive and negative. Two charged particles at rest and bearing the same kind of charge exert a mutually repulsive force, whereas two particles at rest but with opposite charge exert a mutually attractive force. With charges $q_{i}$ and $q_{j}$ separated by the distance $r_{i j}$, the foregoing is summarized in Coulomb's law, which for the vacuum and in ESU units is given by:

$$
\vec{F}_{i j}=q_{i} q_{j} \cdot \hat{r}_{i j} /\left(r_{i j}\right)^{2} \text {, }
$$

where $\hat{r}_{i j}$ is the unit vector in the direction of $\vec{r}_{1 j}$. If we consider a static distribution of charges, we are able to calculate the force that they would exert on a given test charge $q_{0}$ in a fixed location.

$$
\vec{F}_{0}=\sum_{j} \frac{q_{0} q_{j}}{\left(r_{0 j}\right)^{2}} \hat{r}_{0 j}
$$

We call $\vec{F}_{0}$ the electric field intensity or simply the electric field arising from the sources $q_{j}$ and denote $1 t$ by $\vec{E}$. Incidentally, this is not an operational definition of $\vec{E}$, since $q_{0}$ would, in fact, disturb the static configuration of $q_{j}$. Some physicists, in a self-stultifying attempt to be operationalistic, produce the non-physical, 
non-sense formula:

$$
\vec{E}=\lim _{q \rightarrow 0} \frac{\vec{F}}{q}
$$

our appraisal of this is indicated by our not bothering to number it. In general, if the system of point charges is sufficiently dense, we conventionally treat it as a continuous charge distribution and replace the sum of (7.2) by an integral. Then we have the electric field intensity at $(x, y, z)$ produced by a charge distribution over the region $\int_{V} d x^{\prime} d y^{\prime} d z^{\prime}$ given by:

$$
\vec{E}=\int_{v} \frac{\rho\left(x^{\prime}, y^{\prime}, z^{\prime}\right) \hat{r} d x^{\prime} d y^{\prime} d z^{\prime}}{r^{2}},
$$

where $\rho$ is the charge density. We now define the flux of $\vec{E}$ through an arbitrary closed surface in terms of the total charge enclosed by that surface as:

$$
\int_{s} \vec{E} \cdot d \vec{a}=\pi \pi \int_{v} \rho d v \text {, }
$$

which is known as Gauss's law. Then by the divergence theorem, we have:

$$
\nabla \cdot \vec{E}=4 \pi \rho \text {, }
$$

which is one of Maxwell's equations.

It is easily shown that the nature of the electrostatic field is such that it may be expressed as the gradient of a scalar field; that is:

$$
\vec{E}=-\nabla \phi
$$

Inserting (7.6) in (7.5), we have:

$$
\nabla^{2} \phi=-4 \pi \rho
$$

which is known as Poisson's equation. It should be noted that whereas (7.5) holds for all electric flelds, in vacuo, 
(7.7) is only applicable in the case of an electrostatic field. That is to say, if a frame of reference can be found relative to which all of the charges are at rest, then (7.7) may be applied.

There is a mass of experimental evidence, including the very existence of matter as we know it, that the charge $q$ is a relativistic invariant. Hence, (7.4) may be evaluated in any inertial frame. That is:

$$
\int_{S(t)} \vec{E} \cdot d \vec{a}=\int_{\zeta(\vec{E})} \overrightarrow{\vec{a}} \cdot d \overrightarrow{\vec{a}}
$$

A fortiori, $(7.5)$ must be relativistically covariant. In fact, we must bear in mind a certain restriction on (7.8), namely that the Gaussian surface $s$ at time $t$ encloses the same charge as $\bar{S}$ at $\bar{E} \neq t$. On the other hand, $(7.5)$ is a local relationship in which such a consideration could not arise.

Let us pause to consider the implications of charge invariance. Since charges produce flelds, the invariance of charge must have an effect on the transformation properties of the electric field. Suppose that we have two parallel sheets of charge of density $+\rho$ and $-\rho$, in the frame $k$, which are of negligible thickness and whose surface areas are very large relative to the distance between them, so that the field between them may be regarded as effectively uniform. Let us coordinatize $\mathrm{K}$ so that both sheets are parallel to the $x y$-plane. Now the magnitude of such a field is simply $4 \pi \rho$. A second 
frame $\vec{K}$ is moving in the $-\mathrm{x}$-direction with respect to $\mathrm{K}$. The length of the sheet must undergo a relativistic contraction with respect to the 'moving' frame. However, the total charge must remain constant. Hence, the charge density must be increased by:

$$
\rho \rightarrow \bar{\rho}=\left(1-v^{2} / c^{2}\right)^{-1 / 2} \rho
$$

The only component of $\overrightarrow{\mathrm{E}}$ between the sheets of charge is in the $z$-direction. Hence, by applying Gauss's law(7.4) in $\overline{\mathrm{K}}$, we have:

$$
\bar{E}_{\bar{z}}=4 \pi \bar{\rho}=\left(1-v^{2} / c^{2}\right)^{-1 / 2} 4 \pi \rho
$$

On the other hand, if the sheets of charge were oriented parallel to the $y z-p l a n e$ of $K$, the surface would not have been subject to relativistic contraction. In this case, there would be a uniform field with one non-vanishing component $E_{X}$, and with respect to $\bar{K}$ we would have:

$$
\bar{E}_{\mathrm{X}}=E_{\mathrm{X}}
$$

The example that we have employed is that of an extremely simple charge configuration. However, as we intimated earlier, if the field is of objective significance it must be independent of its sources. Hence, under any circumstances, regardless of sources, the principle of relativity should enable us to determine the nature of the field in any inertial frame, given its description in a particular frame.

Let us, once more, take a 'stationary' frame $K$, coordinatized by the system $S$ such that a point charge $Q$ 
is stationary at the origin of $S$. Let $\vec{r}$ be a radius vector in $S$. It follows as an elementary consequence of $(7.4)$ that the field of $Q$ is given by:

$$
\vec{E}=Q / r^{2}
$$

Let us restrict our considerations to the field in the $\mathrm{xz}$-plane of $\mathrm{S}$. Resolving $\overrightarrow{\mathrm{E}}$ into its components in this plane, we have:

$$
\begin{aligned}
& E_{x}=\frac{Q}{r^{2}} \cos \theta=\frac{Q x}{\left(x^{2}+z^{2}\right)^{3 / 2}} \\
& E_{z}=\frac{Q}{r^{2}} \sin \theta=\frac{Q z}{\left(x^{2}+z^{2}\right)^{3 / 2}}
\end{aligned}
$$

Now consider a 'moving' frame $\vec{k}$ having the velocity $\vec{V}$ in the -x-direction with respect to $Y$ and coordinatized by $\bar{s}$ such that $x=\bar{x}=0$ at $t=\bar{t}=0$. We now express the components $E_{X}$ and $E_{z}$ in $\bar{K}$ at $\bar{t}=0$. From (5.10), (7.10), (7.11) and (7.13), we have:

$$
E_{x} \rightarrow \bar{E}_{x}=E_{x}=\frac{\left(1-v^{2} / c^{2}\right) Q \bar{x}}{\left[\left(1-v^{2} / c^{2}\right)^{-1} \bar{x}^{2}+\bar{z}^{2}\right]^{3 / 2}}
$$

and, $E_{z} \rightarrow \bar{E}_{z}=\left(1-v^{2} / c^{2}\right)^{-1 / 2} E_{z}=\frac{\left(1-v^{2} / c^{2}\right)^{-\frac{1}{2}} Q \bar{z}}{\left[\left(1-v^{2} / c^{2}\right)^{-1} \bar{x}^{2}+\bar{z}^{2}\right]^{3 / 2}}(7.15)$ Dividing (7.15) by (7.14), we have:

$$
\overline{\mathrm{E}}_{\mathrm{z}} / \overline{\mathrm{E}}_{\mathrm{x}}=\overline{\mathrm{z}} / \overline{\mathrm{X}}
$$

Thus, the direction of the field vector $\overrightarrow{\vec{E}}$ must be in the direction of $\vec{r}$, which is the radius vector from 2 at $t=\bar{t}=0$.

It follows from the above considerations that at an 'observation point' in $\bar{K}$, say ten miles from the origin of $\bar{s}$, the field will 'appear' to be emanating from $\bar{o}$ at $\bar{t}=0$. Since we cannot have instantaneous action at 
a distance, it follows that the field at the observation point in $\bar{K}$ was determined by its source at a time prior to $\bar{t}=0$. We shall refer to the significance of this shortly.

Continuing to work in two dimensions, we now make a purely notational change by suppressing the bars of the $\bar{K}$ variables. In effect, we are now treating $\bar{K}$ as a 'stationary' frame in which a point charge is moving in the $+x-d i r e c t i o n$ with velocity $\vec{V}$. However, since the change is purely notational, the form of $(7.14)$ and (7.15) is unmodified. We now compute the strength of the field in the new 'stationary' frame $K$, our former $\bar{K}$. From $(7.14)$ and $(7.15)$, we have in the new notation:

$$
|\vec{E}|=\left[\left(E_{x}\right)^{2}+\left(E_{z}\right)^{2}\right]^{1 / 2}=\frac{\left(1-v 2 / c^{2}\right)^{-1 / 2} Q\left(x^{2}+z^{2}\right)^{1 / 2}}{\left[\left(1-v^{2} / c^{2}\right)^{-1} x^{2}+z^{2}\right]^{3 / 2}}(7.17)
$$

By (7.16), we know that the direction of the field is

given by the radius vector from the instantaneous position of the point charge to the observation point. Since the origin 0 is arbitrary, this result is general. However, there is a significant difference between the new field $\vec{E}$ and that of $(7.6)$. Taking the curl of $(7.6)$, we have:

$$
\nabla \times \vec{E}=-(\nabla \times \nabla \phi) \equiv 0
$$

This is a necessary consequence of the radial symmetry of the field of a stationary charge. We call such fields conservative, and it is this that permits us to express them as the gradient of a scalar. In general, for any 
electrostatic field:

$$
\oint \vec{E} \cdot d \vec{s}=0
$$

However, inspection of (7.17) reveals that the values of the physical coordinates $X$ and $Z$ are no longer formally interchangeable. In short, our new field, while still radial, is no longer radially symmetrical. In particular, the field at right angles to the $x$-axis, the direction of $\vec{V}$, will be stronger than the field parallel to $\vec{V} \cdot(7.19)$ will no longer hold and, accordingly, no static charge configuration could produce it. In differential form:

$$
\nabla \times \vec{E} \neq 0
$$

or, $\oint \vec{E} \cdot d \vec{s} \neq 0$

At first sight, the principle of relativity seems to have been violated. We arrived at the condition (7.20) via a transformation from one inertial frame to another. Surely, the field equations should be covariant under such a transformation. For the moment, we shall content ourselves with a partial resolution of this problem. Firstly, the situation is somewhat analogous to the issue of the proper time interval which was discussed in Chapter V. The non-covarlance of (7.19) is based on the selection of a privileged point charge, namely one that is at rest in the 'stationary' frame. If we considered a similarly distinguished point charge in the 'moving' frame, we would find that the effect is completely reciprocal between the two frames. Thus, the discrepancy 
between ( 7.19$)$ and $(7.21)$ does not provide a basis for distinguishing one inertial frame from another or, in particular, for distinguishing a privileged frame. On the other hand, it is true that we have contrived a velocity dependent force field. The velocity dependent factor must be worked into the field equations to make them covariant. The study of the covariance properties of the electrostatic field does not reveal a direct violation of the principle of relativity, which would falsify our thesis since the one is formal while the other is physical, but does reveal that electrostatics is an incomplete theory. Ultimately, we shall find that a complete relativistic theory must have as its primary object the electromagnetic field, of which the electrostatic field is a very special case. However, we must first return to a piece of unfinished business.

We noted in our last physical example that at an observation point ten miles from the point charge, the field vector will be in the radial direction. This implied that the source had produced a field prior to its reaching $O$ at $t=E=0$. Suppose, now, that we have a frame of reference $K$ in which the point charge is in uniform motion in the $+x$-direction. When it reaches the arbitrary origin 0 it is brought instantaneously to rest at $t=0$. At the observation point $(x, z)$ at $t=0$, the field must be given by $(7.17)$, i.e. the field of a moving 
charge at the origin. However, at any other observation point, at a distance $s<$ ct from 0 , the field will be that of a motionless charge. Hence, there must be a sudden change in both the magnitude and direction of the field. Let us take two observation points: $P_{1}\left(x_{1}, z_{1}\right)$ in the field of the stationary charge and $\mathrm{P}_{2}\left(\mathrm{x}_{2}, z_{2}\right)$ in the field of the moving charge. The direction of $\vec{E}$ at $P_{1}$ is measured at the same time as the direction of $\vec{E}$ at $P_{2}$. If $\theta$ is the angle which $\vec{E}_{(1)}$ makes with the $x$-axis and $Q$ is the angle which $\vec{\Xi}_{(2)}$ makes with the $x$-axis, then a shrewd application of Gauss's law, which we need not explain, will yield the relation:

$$
\tan \phi=\left(\tan \theta X 1-v^{2} / c^{2}\right)^{-\frac{1}{2}}
$$

Thus, to conform to the principle of relativity, it is necessary that the direction of the electric field underso a precession determined by a change of velocity relative to an inertial frame. At a later point in this chapter, we shall find that such effects have profound significance in the formulation of the Principle of Special Relativity. We have, so far, considered the force field generated by a uniformly moving charge. We now consider the case of the force exerted on a charge which moves uniformly in the field of an existing charge configuration. In the case of an electrostatic field, the answer is virtually at hand. Given a point charge with velocity $\vec{v}$ with respect to $k$, transform to the rest frame. of the 
charge $\bar{K}$. The force on the point charge will be given by:

$$
\vec{F}=q \vec{E}
$$

We then find the transformation law for the force. We shall omit the mathematical computation, since we have not yet introduced the convenient four-vector formalism in this chapter. However, we state the result:

$$
\overrightarrow{\vec{F}} \rightarrow \vec{F}=g \overrightarrow{\mathbb{E}}
$$

In words, the force of an electrostatic field on a moving charge is independent of the velocity of the charge. Relativistic factors enter the computation of the components of $\vec{F}$ and $\vec{E}$, but they cancel out to give (7.24). To consider the force on a charge which is moving in the field of a non-static configuration, it is necessary to turn at last to the magnetic field. We shall begin by considering the idealization of the magnetic field which is produced by steady currents and constant charge densities. This aspect of electrodynamics is often, in analogy with electrostatics, given the unfortunate title of magnetostatics which, at least from the Amperian point of view, is actually a contradiction in terms. It is a well-established empirical fact that moving charges exert forces on each other whose magnitude is velocity dependent and whose direction is at right angles to the velocity and a unique spatial direction. It is possible to represent this relatively complicated force by utilizing a vector field $\vec{B}$ given by: 


$$
\vec{F}=(q / c)(\vec{V} \times \vec{B})
$$

For example, current-carrying parallel wires will attract or repel each other depending on whether the currents are respectively in the same or opposite directions. When a charge density $\rho$ is in motion it constitutes a flow of charge. We define the flow density or current density by:

$$
\vec{J}=\rho \vec{V}
$$

The total charge passing through a surface per unit of time is the electric current, given by:

$$
I=\int_{s} \vec{J} \cdot d \vec{a}
$$

Consider an inertial frame $K$ with a currentcarrying wire which is aligned with the $x$-axis of $S$. The positive charges are fixed in the wire while some of the negative charges are moving in the +x-direction with velocity $\vec{V}$. Let us suppose that $\rho_{+}=-\rho_{-}$, so that the wire is electrically neutral and generates no electric field. At a distance $r$ from the wire, a negative point charge $q$ is moving parallel to the wire with a velocity $\vec{V}_{0}$ in the $+x-d i r e c t i o n$. Consequently, the moving charge must be deflected towards the wire under the action of the magnetic force given by $(7.25)$. We now transform to $\bar{K}$ in which $g$ is at rest. The charge can no longer experience a magnetic force since its velocity is now zero. However, the principle of relativity requires that the charge still be deflected towards the wire. We assume that there must be an electric force acting on the charge. With respect 
to $\overline{\mathrm{K}}$, the wire is now moving in the $-\mathrm{x}$-direction, with the velocity $\vec{v}_{0}$. By $(7.9)$, the charge densities $\rho_{+}$and $\rho_{-}$ must undergo a relativistic change. The positive charges are now moving with velocity $\vec{V}_{0}$ in the -x-direction, while the negative charges are moving with velocity $\vec{v}-\vec{v}_{0}=\vec{v}_{1}$ in the -x-direction. Hence, the new charge densities with respect to $\bar{K}$ are given by:

$$
\left.\begin{array}{l}
\bar{\rho}_{+}=\rho_{+}\left(1-v_{0}^{2} / c^{2}\right)^{-1 / 2} \\
\bar{\rho}_{-}=\rho_{-}\left(1-v_{1}^{2} / c^{2}\right)^{-1 / 2}
\end{array}\right\}
$$

Clearly, the wire is now positively charged and produces an electric field $\vec{E}$ which exerts a force on the stationary charge towards the wire. The scientific reader may be disturbed by the qualitative nature of the foregoing account. However, we merely wish to interpret in an intuitive way the interrelationship between the electric and magnetic fields from the standpoint of the principle of relativity.

We now state the general relationship between the magnetic field $\vec{B}$ and the current density $\vec{J}$. The total current enclosed by a curve $C$ is equal to the flux of $\vec{J}$ through a surface which spans $C$. Thus:

$$
\int_{c} \vec{B} \cdot d \vec{s}=\frac{4 \pi}{c} \int_{s} \vec{J} \cdot d \vec{a}
$$

3y Stokes' theorem, we have:

$$
\int_{C} \vec{B} \cdot d \vec{s}=\frac{4 \pi}{C} \int_{S}(\nabla \times \vec{B}) \cdot d \vec{a}
$$

Whence:

$$
\nabla \times \vec{B}=\frac{4 \pi}{c} \vec{J}
$$


Finally, the magnetic field is determined by the further condition:

$$
\nabla \cdot \vec{B}=0
$$

That is to say, unlike the electric field, the magnetic field has no sources, i.e. there are no magnetic charges. We may now summarize the basic equations of electrostatics and magnetostatics:

$$
\left.\begin{array}{l}
\nabla \cdot \vec{E}=4 \pi \rho \\
\nabla \cdot \vec{B}=0 \\
\nabla \times \vec{E}=0 \\
\nabla \times \vec{B}=\frac{4 \pi}{c} \vec{J}
\end{array}\right\}
$$

However, when there are changes in the currents and charges, the time derivatives of the fields must be included. Then the general equations of the electromagnetic field are given by inaxwell's equations:

$$
\left.\begin{array}{l}
\nabla \cdot \vec{E}=4 \pi \rho \\
\nabla \cdot \vec{B}=0 \\
\nabla \times \vec{E}=-\frac{1}{c} \frac{\partial \vec{B}}{\partial t} \\
\nabla \times \vec{B}=\frac{1}{c} \frac{\partial \vec{E}}{\partial t}+\frac{4 \pi}{c} \vec{J}
\end{array}\right\}
$$

The field equations are, of course, to be supplemented by the force law:

$$
\vec{F}=q \vec{E}+(q / c)(\vec{V} \times \vec{B})
$$

We conclude this part of the presentation with two further elementary points. Just as the electrostatic field could be expressed in terms of the gradient of a scalar potential, so the magnetic field may be expressed 
in terms of the curl of a vector which is called the vector potential:

$$
\vec{B}=\nabla \times \vec{A}
$$

secondly, we wish to comment on the significance of the first term on the right hand side of the last of the equations $(7.34)$.

Since charge is invariant, the amount of charge leaving an enciosed volume must precisely equal the decrease in charge in that volume. This appears to be a tautology but actually has physical significance. Accordingly, we may express the relationship between current density and charge density by a so-called equation of continuity. Incidentally, while charge invariance is a sufficient condition, it is not necessary for charge conservation.

$$
\nabla \cdot \vec{J}=-\frac{\partial \rho}{\partial t}
$$

But in the case of a 'stationary' current, we have:

$$
\nabla \times \vec{B}=\frac{4 \pi}{c} \vec{J}
$$

In the case of a varying charge distribution, by (7.37):

$$
\nabla \cdot \vec{J} \neq 0
$$

However, the divergence of the curl of an arbitrary vector vanishes identically. Hence, by (7.33), we have:

$$
\nabla \cdot(\nabla \times \vec{B})=\frac{4 \pi}{c} \nabla \cdot \vec{J} \equiv 0
$$

obviously, (7.39) is incompatible with (7.37) and, since we cannot doubt the truth of ( 7.37$)$, it follows that (7.33) cannot hold for varying currents. In fact, even - $\nabla \cdot \vec{J}=0^{\prime}$ is a gross idealization in magnetostatics. In any 
event, it is clear that another term must be added to (7.33) in the physically real case of varying currents. According to Faraday's law, a changing magnetic field is accompanied by an electric field.

$$
\nabla \times \vec{E}=-\frac{1}{c} \frac{\partial \vec{B}}{\partial t}
$$

It is natural to assume, and this is strictly heuristic, that a changing electric field 'induces' a magnetic field. Thus, by analogy with $(7.34)$, we rewrite $(7.33)$ as:

$$
\nabla \times \vec{B}=\frac{1}{c} \frac{\partial \vec{E}}{\partial t}+\frac{4 \pi}{c} \vec{J}
$$

This is not necessarily a physically true law, but by virtue of (7.37) it does satisfy the identity (7.39), so that the contradiction is resolved. The new term $\frac{1}{c} \frac{\partial \vec{E}}{\partial t}$ is called the displacement current. Faraday failed to discover the displacement current, since for relatively slow changes in the field, the convection (and conduction) currents alone suffice to account for observed magnetic effects. After a lengthy discussion, which was trying for the philosopher and excruclatingly boring for the physicist, we are now ready for the payoff which begins with the rewriting of Classical Electrodynamics in manifestly Lorentz covariant form. It is well known to the student of elementary vector analysis that when one performs a coordinate transformation, for example from Cartesian to polar coordinates, it is not only the vectors but also the vector operators that must be appropriately modified. Similarly, in the four-vector formalism, it is 
normal to expect the four-vector operators to differ from their three-vector counterparts. However, so long as we continue to use Minkowski coordinates, the modification is the most simple and natural one possible. We simply replace $\nabla$ by $\square$ given by:

$$
\square=d f \cdot \frac{\partial}{\partial x^{1}}+\frac{\partial}{\partial x^{2}}+\frac{\partial}{\partial x^{3}}+\frac{\partial}{\partial x^{4}}
$$

Similarly, the four-vector counterpart of the Laplacian, known as the d'Alembertian, is given by:

$$
\square^{2}=d f \cdot \frac{\partial^{2}}{\partial x^{1} \partial x^{1}}+\frac{\partial^{2}}{\partial x^{2} \partial x^{2}}+\frac{\partial^{2}}{\partial x^{3} \partial x^{3}}+\frac{\partial^{2}}{\partial x^{4} \partial x^{4}}
$$

We shall, however, make use of the comma notation to signify four-dimensional differential operations. Thus, the four-divergence of an arbitrary vector $V^{\mu}$ is given by:

$$
\square V^{\mu}=d f . \quad V^{\mu}, \mu
$$

The equation of continuity (7.37) may be rewritten as:

$$
\nabla \cdot \vec{J}+\frac{\partial \rho}{\partial t}=\square J^{M} \equiv J^{M}, \mu
$$

(7.44) is Lorentz covariant provided that $\mathrm{J}^{\mathrm{M}}$ is a fourvector. Now $\mathrm{J}^{\mu}$ may be written as:

$$
J^{M}=(\vec{J}, i c \rho)=(\rho \vec{v}, i c \rho)=\rho\left(1-v^{2} / c^{2}\right)^{1 / 2} U^{M} \text { (7.45) }
$$

$U^{\mu}$ is the already familiar four-velocity, and we gather from (7.9) that:

$$
\rho\left(1-v^{2} / c^{2}\right)^{1 / 2}=\rho_{0}
$$

is the proper charge density. Hence, $J^{M}$ is the four-current density given by:

$$
J^{M}=\rho_{0} U^{M} \text {, }
$$

which is manifestily Lorentz covariant. $J^{\mu}$ is a useful 
theoretical quantity, since $J^{i}$ gives the current density while $J^{4}$ gives the charge density.

It is well known in Classical Electrodynamics that whereas the vector potential $\vec{A}$ determines the field $\vec{B}$, the converse does not hold. Since the curl of a gradient is identically zero, it is always permissible to add such a quentity to $\vec{A}$ without affecting $\vec{B}$. However, to preserve the consistency of the equations, it is necessary to make a corresponding.chamge in the scalar potential. Thus, we have the so-called gauge transformation:

$$
\left.\begin{array}{l}
\vec{A} \rightarrow \vec{A}^{*}=\vec{A}+\nabla \psi \\
\phi \rightarrow \phi^{*}=\phi-\frac{\partial \psi}{\partial t}
\end{array}\right\}
$$

$\psi$ is known as the gauje function. If it is made to satisfy the condition:

$$
\nabla \cdot \vec{A}+\frac{1}{c} \frac{\partial \theta}{\partial t}=0
$$

which is called the Lorentz gauge, there follows:

$$
\nabla^{2} \vec{A}-\frac{1}{c^{2}} \frac{\partial^{2} \vec{A}}{\partial t^{2}}=-\frac{4 \pi}{c} \vec{j}
$$

and, $\nabla^{2} \phi-\frac{1}{c^{2}} \frac{\partial^{2} \phi}{\partial t^{2}}=-4 \pi \rho$

Defining the four-vector potential $A^{M}$ by:

$$
A^{M}=d f . \quad(\vec{A}, i \phi)
$$

we may combine $(7.50)$ and $(7.51)$ in the form:

$$
A^{M}, v V=-\frac{4 \pi}{C} j^{M}
$$

Given the values of $\vec{A}$ and $\phi$ in an inertial frame $K$, the corresponding values of $\vec{E}$ and $\vec{B}$ are given by:

$$
\begin{aligned}
& \vec{E}=-\nabla \varphi-\frac{1}{c} \frac{\partial \vec{A}}{\partial t} \\
& \vec{B}=\nabla \times \vec{A}
\end{aligned}
$$


Let us now see how $(7.54)$ and $(7.55)$ may be combined by way of the four-vector potential. By (7.52), $(7.54)$ and (7.55), we may work out a sample component of $\vec{E}$ and one of $\vec{B}$.

$$
\left.\begin{array}{rl}
-i E_{1} & =A^{4}, 1-A^{1}, 4 \\
B_{1} & =A^{3}, 2-A^{2}, 3
\end{array}\right\}
$$

In general, we define the electromagnetic field tensor:

$$
F^{M V}=A^{2}{ }_{1 M}-A^{M}, V
$$

where $\mathrm{F}^{\mathrm{MV}}$ is given by the matrix:

$$
F^{M V}=\left(\begin{array}{cccc}
0 & B_{3} & -B_{2} & -i E_{1} \\
-B_{3} & 0 & B_{1} & -i E_{2} \\
B_{2} & -B_{1} & 0 & -i E_{3} \\
i E_{1} & i E_{2} & i E_{3} & 0
\end{array}\right)
$$

Faxwell's two sets of equations may now be written as a single 'source' equation and a single 'internal' equation.

$$
\left.\begin{array}{l}
F^{\mu v}, V=\frac{4 \pi}{c} J^{\mu} \\
F^{\mu \nu, \sigma}+F^{v \sigma}, \mu+F^{\mu \mu}, V=0
\end{array}\right\}
$$

So far we have shown that Maxwell's equations assume a simple and elegant form in which their Lorentz covariance is manifest. Moreover, we perceive that the peculiarities of the field $\vec{B}$, in particular its being a so-called axial vector, are simply consequences of its actually forming part of an antisymmetric tensor. Moreover, in the course of our presentation, we have attempted to show that the Lorentz covariance of $(7.58)$ 
has a physical basis of which the tensor formalism is the mere expression. A mathematician confronted by ( 7.58$)$ needs merely to be told that they are four-tensor equations to be able to assert their Lorentz covariance. However, that $(7.58)$ represents a real field is another matter. To know this, one must understand the physical basis which justifies the claim that $(7.58)$ are laws of nature. It is not the case that one can ascribe geometrical properties to the representations of physical quantities ad libitum. That linear momentum is a polar vector, that angular momentum is an axial vector are not conventions of Classical Mechanics but the mathematical representation of physical reality. By the same token, that the electromagnetic field is an antisymmetric space-time tensor is not a formal thesis of mathematics but an expression of 'the way things are'.

our next step is to formulate the force law in terms of the electromagnetic field tensor. We shall find that this has particular philosophical significance. We remind the unmathematical reader that a four-tensor equation that holds in one inertial frame must hold in every inertial frame. (This will be generalized in Chapter VIII, where we introduce Riemannian tensors.) To construct a tensor equation, a common stratagem is to choose a frame of reference, in this context inertial, in which it acquires its simplest form. It is then guaranteed 
to hold in any other inertial frame. Our task, now, is to determine the force exerted on a moving charge in the electromagnetic field $F^{\mu \nu}$. Following the aforementioned stratagem, we choose a frame $K$ in which the charge is instantaneously at rest. Let $\vec{E}$ be the electric field intensity at the location of the charge in $K$. It follows from (6.37) that the four-force exerted on the charge $q$ must be:

$$
F^{\mu}=(q \vec{E}, 0)
$$

The four-velocity of the charge in $K$ is given by:

$$
U^{\mu}=(0,0,0, i c)
$$

We now form the quantity:

$$
F^{\mu \mho} \cup=\text { ? }
$$

The only non-vanishing component of $U^{V}$ is $U^{4}$. Hence, (7.61) reduces to:

$$
F^{M+} U^{4}=\text { ? }
$$

Referring to the matrix array $(7.57 a)$ of $F^{\mu v}$, we may write:

$$
F^{\mu U_{U}}=-i c\left(i E_{1}, i E_{2}, i E_{3}, 0\right)=c\left(E_{1}, E_{2}, E_{3}, 0\right)(7.63)
$$

By $(7.59)$ and $(7.63)$, we have:

$$
(\mathrm{q} / \mathrm{C}) \mathrm{F}^{\mathrm{M}} \mathrm{U}^{4}=\mathrm{F}^{\mathrm{M}}
$$

In general, therefore:

$$
(q / c) F^{\mu v} U^{v}=F^{\mu}
$$

The reader may verify that by letting the indices run from 1 to 3, the familiar Lorentz force equation is obtained. With $\mu=V=4$, the equation vanishes identically. However, we are not interested in the classical 
form of the Lorentz force, but directly in (7.65). The reader will recall that our definition of a fundamental law precluded it from containing terms which depend essentially on a particular frame of reference. It was for this reason that the laws of sound propagation could not possibly be regarded as fundamental. But, by the same token, we would be obliged to exclude the force equation of electrodynamics, since it contains a velocity dependent part which depends essentially on the frame of reference. one might even go so far as to say that the classical Lorentz force equation is only covariant under a pure coordinate transformation, in particular, a rotation of the spatial coordinates, since the magnetic force (although not the field) could be 'transformed away' by a Iorentz frame transformation. On the other hand, the tensor equation (7.65) contains the four-velocity $U^{\mu}$ which has the same value, i.e. norm, in all inertial frames. It is, of course, true that the spatial components do not vanish in every frame, but the values of the components are merely incidental just as in the case of three-vectors. We conclude, therefore, that the four-dimensional formalism and, in particular, the combined electromagnetic field tensor is not merely a more elegant way of representing electrodynamics but the only philosophically acceptable way.

Such considerations, however, do not apply to all 
tensorial laws. One can learn little from an examination of the mathematical formalism of a theory, per se. One must also understand the physics which it purports to represent. The distinction between $\vec{E}$ and $\vec{B}$ in Maxwell's equations is frame dependent and can, accordingly, have Iittle to do with the structure of the global patterns of nature. Such considerations add even further support to our distinction between a Galilean frame and a Lorentz frame, namely that the latter must include some sort of clock, which, in the former is regarded as a test instrument, ancillary to the spatial frame of reference. our treatment, to this point, has merely scratched the surface of the intriguingly rich theory of electrodynamics. In particular, we have said nothing of the action of the electromagnetic field on matter. In the Appendix we rapidly cover certain more advanced topics, particularly by taking into account the mechanical mass of our point charges. However, we have exposed enough of the theory to deal in a precise fashion with the Principle of special Relativity and its connection with Lorentz covariance.

In the first place, it is clear that the restricted Lorentz transformation $L_{S}$ is a subgroup of the complete group and is, moreover, a relativity group of electrodynamics. The inhomogeneous spatial rotation group $\mathrm{R}_{13}$ is a subgroup but not a relativity subgroup. 
When dealing with the Galilean group, we pointed out that the general Galilean transformations were the realization of the direct product $R_{i 3} \times G_{S}$. One would expect that the general Lorentz transformation could be represented by the direct product $\mathbb{R}_{13} \times L_{s}$. However, such is not the case. The proof, which is not difficult but too long to include here, is given by [iller(1952, pp. 118ff.). Specifically, if the element of $R_{13}$ is the identity element, then the resultant of two successive transformations:

$$
x^{M} \rightarrow \bar{x}^{M}=\alpha_{\text {pur }} x^{v} \text { and } \bar{x}^{M} \rightarrow \overline{\bar{x}}^{M}=\alpha_{\mu v} \bar{x}^{v}
$$

which is given by:

$$
x^{\mu} \rightarrow \overline{\bar{x}}^{\mu}=\bar{\alpha}_{\mu \rho} \alpha_{\rho v} x^{v}=\overline{\bar{\alpha}}_{\mu v} x^{v}
$$

is not, in general, a Lorentz transformation without rotation.

In simple terms, if $s$ and $\bar{s}$ are fixed systems of coordinates of $K$ and $\bar{K}$ with parallel axes, and $\bar{S}$ is the coordinatization of $\overline{\bar{K}}$ which differs from $\bar{K}$ by a restricted Lorentz transformation, then the image of an arbitrary vector $\overrightarrow{\mathrm{V}}$ in $\overline{\overline{\mathrm{K}}}$ will not, in general, be parallel to $\overrightarrow{\mathrm{V}}$. This is nothing but a manifestation of the Thomas precession effect which we have already mentioned. Furthermore, it may be shown that this effect is independent of the coordinatization. That is to say, it is a time dependent effect. Hence, to preserve the content of the fundanental equations of electrodynamics, it is necessary that the inertial frames undergo spatial rotations. Of course, these 
need not be continuous rotations, in which case the frames would not be inertial. If we think of an abstract vector space in which inertial frames are moving uniformly in all directions, then the transformation (7.67) would not relate $a k$ to a $\overline{\bar{K}}$ according to the laws of vector addition. The angle of precession will depend on the relative velocities and directions of the inertial frames. We have already come across an example of such an effect in (7.22) which revealed that the direction of the field vector $\vec{E}$ depended on the velocity of its source with respect to an inertial frame. Other examples exist to confirm that this is a physical effect which is independent of the coordinatization of the frames of reference. Hience, it must be taken into account in determining the relativity Eroup of jpecial Relativity physics. Actually, this consideration sheds considerable light on the difference between a restricted, in our sense, Galilean transformation and a restricted Lorentz transformation. The extent to which this is ignored by the authors of textiooks on physics is as remarkable as it is deplorable. In elementary texts on Newtonian Wechanics, the restricted Galilean transformation is usually given with the correct, although of ten unstated, implication that the general Galilean transformation is physically the same as the restricted one but with a different coordinatization. It so happens that when three Galilean 
frames are in relative motion with non-parallel relative velocities, the relationship between the first and the third is representable by a restricted Galilean transformation. But when the analogous situation arises vis à vis three Lorentz frames, the relationship between the first and the third will senerally involve a rotation of axes. The unsuspecting student who graduates to special Relativity physics will be presented with the restricted Lorentz transformation, e.s. in Bergmann(1942), and presume that it is simply a particular way of representing the general Lorentz transformation, a perfectly natural but false supposition for which the author of the text is responsible. In fine, when we speak of the restricted lorentz transformation as a subgroup, we imply that the relative velocities of all the elements of the subgroup are parallel, which is a real physical restriction. In the Galilean case, 'restricted' is simply a figure of speech and a formal simplification attained by the most convenient choice of coordinates.

The covariance group of Newtonian Mechanics is $\mathrm{G}_{\mathrm{S}} \times \mathrm{R}_{\mathrm{i} 3} \times \mathrm{T}$, while its relativity group is the subgroup $\mathrm{G}_{\mathrm{S}} \cdot$ The covariance group of special Relativity physics is the complete Lorentz group $I_{C}$. The restricted Lorentz group, in 1ts true meaning, is a relativity group but not the complete relativity sroup. We cannot represent the complete relativity group as $\mathrm{I}_{S} \times \mathrm{R}_{3}$, since this would 
entail that when $L_{S}$ is the identity element, $B_{3}$ is a relativity subsroup when, in fact, it is only a covariance subsroup. 'Thus, we are forced to represent the relativity group of special Relativity physics simply as $I_{r}$, the Eroup of general Lorentz transformations with rotation. Its only relativity subgroup is $I_{S}$. We are now, at last, in a position to formulate the Principle of special Relativity. $\{L\}$ is the set of fundanental laws of a given theory. $\{K\}$ is the set of possible frames of reference. $\{T\}$ is the set of possible frame transformations modelled by elements of the transformation group $I_{r}$.

For any $I \in\{L\}$ and any $t \in\{T\}$, there exist at least two distinct $k_{i}, k_{j} \in\{k\}$, such that 1 w.r.t. $k_{i} \in\{k\}$ $\leftrightarrow t(I)=$ s. $\bar{I}$ w.r.t. $k_{j} \in\{K\}$.

It is interesting to compare this with the formal statement of the Principle of Classical Relativity. In the first place, it is more general since it is, in principle, applicable to any physical theory. In the second place, it clearly shows the necessity for distinguishing between a Galilean frame and a Lorentz frame, since the aistinguished subset $\{I\} \subset\{K\}$ differs in the two principles by virtue of the profound difference between the signification of $\{\mathrm{T}\}$ in the two cases. As to the greater generality of the frinciple of special Relativity, it could be objected that Classical Relativity could have been endowed with the same degree of generality had $\{I\}$ 
been interpreted with the same degree of freedom in the two cases. In fact, in his original paper, Einstein(1905) seems to have been motivated partly by philosophical considerations in applying the Principle of special Relativity to the laws of nature in general. We have already remarked, however, that attempts to broaden the application of Classical Relativity, for example in extending it to electrodynamics, failed to withstand experimental testing. It is true that in virtue of its generality, special Relativity may play a heuristic role in the construction of new theories and the modification of existing ones, but this in no way supports its conventionalistic interpretation. The Principle of special Relativity may be of heuristic value in virtue of its generality, but the basis of its generality is its factuality.

Finally, we should mention the so-called reality conditions on the transformation matrix $\alpha_{\mu r}$, since they must equally be kept in mind in the context of General Relativity. In the form in which we now state them, they depend on the Minkowski coordinates, but a corresponding set exists for real coordinates. It is obvious that $\alpha_{i j}$ must be real since they are the coefficients of a pure spatial rotation. Similarly, $\alpha_{44}$ must be real. It is equal to $\left(1-v^{2} / c^{2}\right)^{-\frac{1}{2}}$, and the square root must be taken as positive. This is the necessary condition for:

$$
\frac{\partial E}{\partial t}>0 ; \frac{\partial t}{\partial E}>0
$$


In words, it is impossible to go backwards in time, which is why the one-parameter group of time translations cannot be part of the relativity group of any theory. We refer to the condition (7.68) as restricting the Lorentz group to the orthochronous subgroup. The reader should be clear concerning the distinction between so-called time reversibility in which time is treated as an independent parameter, and the group of time translations in which time plays the role of a coordinate variable. Time reversibility simply means that it is possible to change the sign of the time parameter throughout an entire theory without altering the significance of that theory. It is a purely mathematical peculiarity which has nothing to do with science fiction. On the other hand, the time translation group entails the possibility of going both ways which is Why it is excluded from the relativity group of every theory.(Cf. Bunge, 1959, Ch. 10.)

To return to the Lorentz matrix, $\alpha_{i_{4}}$ and $\alpha_{4 i}$ must be imaginary. These conditions entail that there is no permissible Minkowski rotation isomorphic to a Lorentz transformation which transforms a spatial axis into a temporal one or vice versa. Moreover, it follows that a time-Iike vector cannot be transformed into a space-like vector or vice versa by a Lorentz transformation. 


\section{CHAPTER VIII}

\section{THE PRINCIPLE OF GENERAL RELAT'IVITY*}

Before dealing specifically with those aspects of General Relativity which pertain to this dissertation, namely the significance of peneral covariance and its relationship to the Principle of General Relativity, we shall permit ourself the luxury of setting the scene in that rather discursive manner that few philosophers are able to resist. Since few authorities appear to agree on even the very meaning of the theory; on whether, for example, it is primarily a theory of gravitation or something much broader, we indicate what the theory signifies to the present writer - a time for considerable temerity.

Let us first review some of our principal points so far. We have shown that both the Principle of Galilei Covariance and the Principle of Lorentz Covariance are strictly formal. It is sheer nonsense to regard them as rival hypotheses or even as rival metatheoretical theorems. From a formal standpoint, each is correct vis à vis its particular referent. The metastatement:" The laws of Newtonian Hechanics' are Galilei covariant," happens to be true. By the same token, the metastatement: "The laws of electrodynamics' are Lorentz covariant," also happens

* The non-mathematical reader should turn to the Appendix before reading the present chapter. 
to be true. There is absolutely no contradiction, implied or explicit, between these metastatements.

on the other hand, we distinguished two principles of relativity: Classical and special. These have been interpreted to be incompatible physical hypotheses. However, contrary to widespread opinion, their incompatibility is not prima facie. The one asserts that the fundamental laws of mechanics have the same content with respect to a distinguished class of reference frames which are called Galilean frames, while the other asserts that the content of the fundamental laws of electrodynamics have the same content in a distinguished class of reference frames which we have chosen to call Lurentz frames. Superficially, the two claims appear to be compatible as, indeed, logically they are. However, the Lorentz frame is a mechanical object and, as such, is subject to the laws of mechanics. The physical coordinates that occur in electrodynamics refer not only to the position of charges, wave fronts, etc. but also to the material particles which compose the frames of reference. Ultimately, therefore, either the laws of mechanics would have to be modified to satisfy the Principle of special Relativity or those of electrodynamics made to satisfy the principle of Classical Relativity. As it happened, the former course led to confirmable consequences while the latter did not. The outcome of the clash between the two hypotheses and 
the consequent overthrow of one was the birth of special Relativity Mechanics. That is to say, the Principle of Special Relativity led to new physics, confirming our contention that its status is that of a physical hypothesis, i.e. an object statement. (Later we shall find a deeper but more formal reason for the necessity of subsuming both mechanics and electrodynamics under a single principle of relativity.)

As orifinally expounded by Einstein, the Theory of General aelativity is a natural extension of the Theory of special Relativity, which was dictated by epistemological considerations. Very simply, since physical processes are indifferent to the method of coordinatizing the space-time manifold, the fundamental laws of physics should be indifferent to an arbitrary choice of coordinates, subject only to some obvious formal restrictions, the most important of which were mentioned in Chapter II. In particular, this entails that the time dependent transformations which model frame transformations need not be restricted to the linear transformations of special Relativity but may be chosen arbitrarily. Hence, the Theory of General Relativity would not distinguish a privileged class of inertial frames. A frame of reference which is accelerating with respect to a Lorentz frame would be just as 'inertial', from the standpoint of General Relativity, as the Lorentz frame, itself. 
While the present writer admits to a strons prejudice against any attempt to derive matters of fact from epistemic premises(Kantianism), there is no doubt that such reasoning may be suggestive, albeit the same could be said of dreams and visions. However, Einstein might have provided an equally heuristic but philosophically more palatable introduction to his new theory on methodolopical grounds rather than epistemological ones. From a methodological standpoint, the most unsatisfactory aspect of any restricted principle of relativity is the occurrence of so-called inertial forces. They are frequently referred to as 'fictitious' forces, with the implication that they have arisen simply from an incorrect choice of reference frame. They provide considerable grist for the mill of the conventionalists, who picture them as mere book-keeping entries which enable us to retain the simple but otherwise valueless $\cdot \vec{F}=m \vec{a} \cdot$. In fact, in this modern age, very few of us have the opportunity to occupy even a quasi-inertial frame for very long and, consequently, have had all too intimate an acquaintance with such 'fictions', which we know to be only too real. Accordingly, Einstein might just as well have satisfied his philosophical comtemporaries by arguing that the recognition in the Theory of special Relativity of a privileged class of inertial frames was unsatisfactory on the ground of its requiring the relezation 
of a large class of physical phenomena to the statis of quasi-occult entities. That is to say, in a methodologically (and ontologically?) sound theory, all phenomena within the scope of that theory should have the same status of reality. Even if we could, unlike the present writer, comprehend the concept of desrees of reality (as in neo-latonism, scholasticism and ixistentialism), one must at least accord the same reality status to entities or phenomena of a sinilar kind. That is to say, it is unintellifible, at least to the present writer, to sneak of some forces' having a greater degree of reality than that of others. Thus, Einstein might have argued that a 'complete' law of nature should incorporate the so-caller inertial forces. Accordinaly, such a law would hold with respect to frames of reference in which inertial forces occur as well as with respect to those in which they do not occur. Admittedly, many of the subsequent remarks of Einstein amounted to a recognition of precisely what we have been saying, although often on the basis of the unclear and unnecessary Hach's Principle. The point we wish to make is not that Einstein was ignorant of the foregoing considerations but merely that they constitute a more 'physical' philosophical motivation than the frinciple of General Covariance, which Einstein, himself, subsequently came to regard as purely formal. The argument based on the Frinciple of General Covariance is possibly more 
appropriate to the doctrine of kinematical relativity which was a more or less crude intuitive precursor to the Theory of General Relativity.

Such considerations lead us naturally to the celebrated Principle of Equivalence. Actually, there are three so-called principles of equivalence connected with General Relativity: viz. the equivalence of gravitational and inertial mass, which has at last been 'committed to the flames' by Bunge(1967a, pp. 207ff.), and the two theorems of General Relativity to the effect that there is at least one coordinate system with respect to which a static homogeneous gravitational field vanishes and that there is at least one reference frame in which the four-acceleration of a test particle vanishes. (Cf. Bunge, ㅇp. cit., pp. 231-232.) The last two may be combined in the more familiar statement of the equivalence of gravitational and inertial fields of force. It is to the latter that we now refer. In popular treatments, this principle is usually misrepresented as asserting that a gravitational field may be transformed away by the selection of a suitably accelerating frame of reference which is attached to a particle which is moving under the influence of such a field, just as inertial forces may be transformed away by the selection of a Lorentz frame. Hence, it is held to follow that gravitational forces must have the very same status as 'fictitious' forces, since all frames 
of reference are equivalent. In fact, of course, this is not true. While it would be possible to attach a reference frame to a single particle whose trajectory is a free-fall, it would not be possible to attach a single frame to two such particles in different regions of space. To be more precise, such a transformation could only hold locally in the case of real, non-uniform gravitational fields.

Even in reputable treatises on the subject of relativity physics, the analogy is often made between the transforming away of a magnetic field and that of a gravitational field. The analogy is extremely weak on two grounds. In the first place, it only works for electrostatic configurations and, in the second place, even in the electrostatic case, the electric field will persist. From the deeper standpoint which postulates the existence of a single electromagnetic field, $F^{\mu v}$, nothing is transformed away in any case but certain components of a tensor. In short, we transform away nothing, but merely give a different representation of the self-identical persisting field.

However, the Principle of Equivalence is a very valuable, albeit qualitative, gulde to the approach to the Theory of General Relativity, so long as it is interpreted to mean only that inertial forces are similar in kind to gravitational forces, insofar as both produce 
accelerations which are independent of the mass of the accelerating body. As such, the principle is strictly true, and this is how we interpret it. Since this is the case, it would seem plausible to attempt to construct laws in which the 'fictitious' terms may be assimilated as special cases of gravitational terms, just as the electrostatic field, with its magically disappearing magnetic aspects, is a special case of the electromagnetic field. Accordingly, a theory of general relativity could be realized in the form of a generally relativistic set of gravitational field equations.

To anticipate the mathematical apparatus which we shall shortly introduce, it is fairly easy to show that if the kinetic energy of a mechanical system be identified with the quadratic form: $T=\frac{1}{2} g_{i k} \dot{x}^{1} \dot{x}^{k}$, the covariant Lagrange equation of motion may be written in the form:

$$
\ddot{x}^{i}+\left\{i_{k}^{i}\right\} \dot{x}^{l} \dot{x}^{k}=F^{i}
$$

where the $\left\{e^{i} k\right\}$, known as the Christoffel symbol, constitute a system of differential coefficients whose significance will be explained later. The classical approach to inertial forces would be to ldentify them with $-\left\{e_{k}^{i}\right\} \dot{x}^{k} \dot{x}^{I}$ which is a term arising from the use of the wrong frame of reference. Alternatively, we may treat all forces as equivalent and write Newton's law as:

$$
F_{C}^{1}=\ddot{x}^{1}
$$

where $F_{C}^{1}=F^{1}-\left\{_{k}^{i}\right\} \dot{x}^{k} \dot{x}^{p}$. However, the combined force $F_{c}^{1}$ 
would obviously depend on the frame of reference. The relativistic approach is, in a sense, the diametric opposite of the latter, insofar as it endeavours to incorporate the external forces in the Christoffel symbol. By virtue of the Principle of Equivalence, such an approach should be successful, at least in the case in which the external forces are exclusively gravitational. Thus, the relativistic approach would be to reduce (8.1) to the form:

$$
\ddot{x}^{i}=-\left\{e^{i} k\right\} \dot{x}^{l} \dot{x}^{k}
$$

which is the equation of motion in the configuration space of the system of interest.

Unfortunately, the program of General Relativity is not realizable by the simple expedient of generalizing the classical equations of motion in this way. Specifically, (8.3) could not possibly serve as a generally covariant law of gravitation, since it would entail that the acceleration of a particle under the influence of a gravitational field would depend quadratically on its velocity in the chosen frame of reference. Moreover, the principal referent of (8.3) is not the field but the point particle. We merely wish the reader to contrast $(8.2)$ with (8.3) to illustrate the method of approach which is adopted in General Relativity, which does not seem to be generally understood.

Two further points which we might have raised in this preamble pertain to the relationship between 
General Relativity and Special Relativity, and the sense in which or the extent to which General Relativity constitutes a 'geometrization' of physics. Both of these are contentious issues, but we shall discuss them only in the context of the more precise presentation which now follows. Again, in recognition that our audience is partly non-mathematical, we begin with a skeletal mathematical exposition which the physicist should skip over. The reader with no training in applied mathematics should begin by rereading Chapter II, Sec. 3. For the moment, the only additional notational convention will be in the formal recognition of the distinction between the covariant and contravariant components of a tensor, which will be indicated by the use of subscript and superscript indices, respectively.

As most readers will know, A Euclidean manifold is defined by the global condition:

$$
(d s)^{2}=d x^{1} d x^{1}
$$

A general Riemannian manifold is one in which the theorem of Pythagoras (8.4) is replaced by the more general condition:

$$
(d s)^{2}=g_{1 k} d x^{i} \mathrm{dx} x^{k}, \quad(1, k=1,2, \ldots, n)(8.5)
$$

In this case, the $x^{i}$ are arbitrary curvilinear coordinates, and the $\delta_{i k}$, known as the metric tensor, may be assigned arbitrarily, subject only to the condition that (8.5) be invariant. If a tensor is attached to every point of a 
Riemannian manifold we have a tensor field, which is an obvious generalization of the vector field of elementary analysis. However, when we attempt to define the derivative of a Riemannian tensor field, we encounter difficulties. Specifically, one cannot compare a tensor(we shall now drop the specification Riemannian which will be taken for granted.) at one point of the manifold with the same tensor at another point. In elementary vector analysis, two vectors are equivalent at different points just in case they have the same Cartesian components. But in the more general Riemannian space, such a definition breaks down. To surmount this difficulty, it is necessary to introduce a specific law of vector displacement which enables us to define the derivative of a tensor. Such a law will enable one to compare a vector $v^{1}$ at $x^{1}$ with the vector $\mathrm{V}^{i}+d \mathrm{~V}^{i}$ at $\mathrm{x}^{i}+d \mathrm{x}^{i}$. The increment in the components of a vector under an infinitesimal displacement is a bilinear function of the components $V^{1}$ and the displacement $d x^{k}$ tangent to the curve along which the displacement takes place. The law of displacement is determined by:

$$
\Gamma_{s t}^{r}=\frac{\partial \bar{x}^{r}}{\partial x^{i}} \frac{\partial x^{j}}{\partial \bar{x}^{3}} \frac{\partial x^{k}}{\partial \bar{x}^{k}} \Gamma_{j k}^{i}+\frac{\partial^{2} \bar{x}^{r}}{\partial x^{j} \partial x^{k}} \frac{\partial x^{j}}{\partial \bar{x}^{3}} \frac{\partial x^{k}}{\partial \bar{x}^{k}}(8.6)
$$

Such an object is known as an affinity or affine connection. The reader will note that its law of transformation is Inear but not homogeneous. However, since the inhomogeneous term is arbitrary, the difference between two affinities 
is a tensor. Moreover, it is obvious that under a Inear transformation (8.6) reduces to the transformation law of a tersor. Thus, the $\Gamma_{j k}$ may be regarded as affine tensors.

It may be shown that the necessary and sufficient condition for the existence of a coordinate system in which the components of a vector are unaltered by an infinitesimal displacement is that the affinity be symmetric in its subscripts. Under this condition, it is possible to choose a local coordinate system in which the affinity vanishes. Such a coordinate system is described as a geodesic system with respect to the given affinity. As in the case of Cartesian tensors, which we have already encountered, a tensor equation is greatly simplified when expressed in terms of geodesic coordinates and, moreover, will hold in any other system.

So far, we have imposed conditions on the manifold similar to those imposed in the latter part of Chapter IV. We now impose the further requirement that the scalar product of two vectors and, \& fortiori, the interval (ds) ${ }^{2}$ be invariant under displacement. This strong requirement renders the system of coefficients $\Gamma{ }_{j k}$ far from arbitrary. It is now called the metric affinity. The condition of the invariance of the scalar product uniquely determines the affine coefficients in terms of the metric tensor. That is to say: 


$$
\frac{d}{d s}\left(g_{i k} v^{i} V^{k}\right)
$$

leads after expansion and various algebraic manipulations to:

$$
\Gamma_{j k}^{i}=\frac{1}{2} g^{2 i}\left(\frac{\partial g_{j l}}{\partial x^{j}}+\frac{\partial g_{\ell k}}{\partial x^{i}}-\frac{\partial g_{i k}}{\partial x^{l}}\right)
$$

For ease of expression, we define the Christoffel symbol of the first kind by:

$$
[i k, l]=d f \cdot \frac{1}{2}\left(\frac{\partial g_{j \ell}}{\partial x^{j}}+\frac{\partial g l k}{\partial x^{i}}-\frac{\partial g j k}{\partial x^{l}}\right)
$$

and the Christoffel symbol of the second kind is given by:

$$
\left\{i_{k}^{j}\right\}=d f . g j l[i k, l]
$$

Whence:

$$
\Gamma_{j k}^{i}=\{j k\}
$$

It is important to note that, contrary to the practice of several elementary expositions, the symbol of affine connection is not simply a different notation for the Christoffel symbol of the second kind.

$$
\text { We now adopt the comma notation to indicate }
$$
ordinary differentiation and the semicolon to indicate covariant differentiation. It is easily shown that the covariant derivative of a contravariant vector is given by:

$$
V_{j j}^{i}=V^{i}, j+\left\{j_{\ell}\right\} V^{l}
$$

Similarly, the covariant derivative of a covariant vector is given by:

$$
V_{i j j}=V_{i, j}-\left\{i_{i}{ }_{j}\right\} V_{l}
$$

The generalization to tensors of arbitrary rank and type follows naturally from (8.12) and (8.13). We also note that in a geodesic system the covariant derivative is 
identical with the ordinary derivative since the affinity and, therefore, the Christoffel symbols vanish in such a system. The covariant derivative of the metric tensor is zero. Unlike ordinary differentiation, covariant differentiation is not commutative, i.e., in general, $T$; Im $\neq \mathrm{T}$; ml , where $T$ is an arbitrary tensor.

All of the foregoing belongs to standard mathematics and has been stated without proof. We do not expect the philosophical reader who is not conversant with higher mathematics to have fully understood all of our exposition, due to its highly compressed character. However, it at least has the virtue of indicating what he needs to know as the barest minimum of mathematics to make sense of the Theory of General Relativity. For such a person, we highly recommenend the leisurely and readable, yet fairly rigorous, treatment of these matters by Schrodinger (1950).

We are now prepared to lay the foundation for the gravitational equations of General Relativity. The n-dimensional Riemannian manifold is now specialized to the four-dimensional space-time manifold. The use of the imaginary coordinate, which enabled us to treat space-time as formally Euclidean, ceases to be of any use and we now abandon it. We let Greek space-time indices run from 0 to 3 and Latin space indices from 1 to 3 . Thus $x^{0}=c t$. We define the Lorentz metric $g_{\mu V}=g_{\mu v}^{(L)}$ by $\operatorname{diag}(1,-1,-1,-1)$. 
We spoke earlier of the disputatious question concerning the relationship between special and General Relativity. While it is not the purpose of this dissertation to consider the finer details of this matter, it is obvious that the two theories are not unrelated. In previous chapters, we have examined the fundamental laws of physics under the assumption that gravitational forces could be neglected. And it is certainly the case that in comparison with electromagnetic forces they are very minute. Consider that a tiny magnet in picking up a piece of iron is able to overcome the gravitational force exerted on the iron by the massive earth. We would assume that in the complete absence of gravitational forces, the laws of special Relativity physics would hold rigorously. Hence, the field equations of General Relativity must entail a global Lorentz metric for gravity free space. This is not simply a heuristic correspondence principle but, in our opinion at least, a theoretical necessity. Since the Lorentz metric is intimately connected with the law of light propagation, in vacuo, a solution of the field equations for gravity free space which did not admit the Lorentz metric globally would require a fundamental revision of the laws of optics and, mutatis mutandis, those of electrodynamics. For this reason, an axiomatization of a high level theory such as General Relativity must be treated with caution. Sometimes the 
heuristic scaffolding may be thrown away when primitive concepts are introduced, but at other times the scaffolding is not merely heuristic but also constitutive. Bunge(1967a, p. 218) is not quite as incisive as we would have liked Vis $\underline{\text { à }}$ Vis his statement concerning the connection between the two theories, but appears to regard it largely, if not wholly, in terms of an heuristic correspondence principle. One thing, at least, is certain. General Relativity is not simply the generally covariant formulation of special Relativity. That is to say, the casting of the laws of special Relativity physics in generaily covariant form does not yield the physical consequences of General Relativity. We shall say a little more on the subject shortly.

An important property of a globally constant metric, Lorentz or otherwise, is that it may be reduced to canonical form throughout the entire manifold. Thus, one can always find a permissible mapping in which the affinity and, a fortiori, the Christoffel symbols are everywhere zero. As we know, in such a system covariant differentiation reduces to ordinary differentiation and is, therefore, commutative. This provides a basis for a sufficient condition for the manifold to admit a globally Lorentz metric. For an arbitrary covariant vector $\mathrm{T}_{\mu}$, we have:

$$
T_{\mu ; \alpha \beta}-T_{\mu ; \beta \alpha}=0
$$


Although the consequence of (8.14) is standard, it is of such great importance that we shall perform the indicated operation step by step for the benefit of the nonmathematical reader. By following each step, he will considerably enhance his appreciation of the tensor notation. We begin with the first term on the left hand side of $(8.14)$.

$$
\begin{aligned}
& T_{\mu ; \alpha \beta}=\left(T_{\mu ; \alpha}\right) ; \beta=\left(T_{\mu ; \alpha}\right), \beta-\left\{\gamma_{\mu \beta}^{\gamma}\right\} T_{\gamma ; \alpha}-\left\{\alpha \gamma \beta T_{\mu j \gamma}\right. \\
& =\left(T_{\mu, \alpha}-\left\{\begin{array}{l}
\gamma \\
\mu \alpha
\end{array}\right), \beta-\left\{q_{\mu \beta}^{\gamma}\right\}\left(T_{\gamma} \alpha-\left\{q_{\gamma \alpha}^{\rho}\right\} T_{\rho}\right)-\left\{\alpha_{\alpha}^{\gamma}\right\}\left(T_{\mu, \gamma}-\left\{\sigma_{\mu}^{\sigma} \gamma\right) T_{\sigma}\right.\right. \\
& =T_{\mu, \beta \alpha}-\left\{\frac{\gamma}{\mu \alpha}\right\}, \beta T_{\gamma}-\left\{\gamma_{\mu \alpha}^{\gamma}\right\} T_{\gamma, \beta}-\left\{\gamma_{\mu \beta}^{\gamma}\right\} T_{\gamma, \alpha} \\
& +\left\{\begin{array}{c}
\gamma \\
\mu \beta
\end{array}\right\}\left\{\begin{array}{c}
\rho \\
\gamma \alpha
\end{array}\right\} T_{\rho}-\left\{\gamma_{\beta}^{\gamma}\right\} T_{\mu, \gamma}+\left\{\begin{array}{c}
\gamma \\
\alpha \beta
\end{array}\right\}\left\{\begin{array}{c}
\sigma \\
\mu
\end{array}\right\} T_{\sigma}
\end{aligned}
$$

By interchanging $\alpha$ and $\beta$ in (8.15) and subtracting, we get af ter relabeling dumm indices:

$$
\begin{aligned}
& T_{\mu ; \alpha \beta}-T_{\mu ; \beta \alpha}=\left\{\begin{array} { c } 
{ \rho } \\
{ \mu \beta }
\end{array} \left\{\left\{\begin{array}{c}
\gamma \\
\rho \alpha
\end{array}\right\} T_{\gamma}-\left\{\begin{array}{r}
\gamma \\
\mu \alpha
\end{array}\right\}, T_{\gamma}-\left\{\begin{array}{c}
\rho \\
\mu \alpha
\end{array}\right\}\left\{\begin{array}{c}
\gamma \\
\rho \beta
\end{array}\right\} T_{\gamma}\right.\right. \\
& \text { Defining: } \\
& +\{\underset{\mu \beta}{\gamma}\}, \alpha T_{\gamma}
\end{aligned}
$$

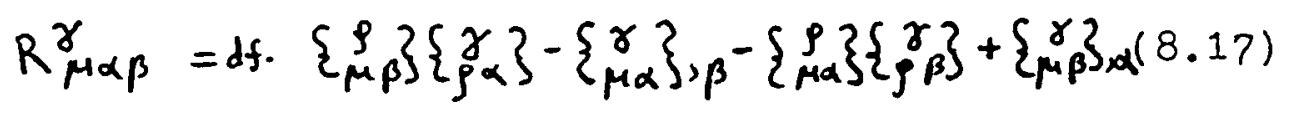

(8.16) reduces to:

$$
T_{\mu j \alpha \beta}-T_{\mu ; \beta \alpha}=R_{\mu \alpha \beta}^{\gamma} T_{\gamma}
$$

since the left hand side of $(8.18)$ is a tensor, it follows that (8.17) is a tensor of the fourth rank. It is known as the Riemann-Christoffel tensor, and in the pure theory of tensor analysis plays the role of the commutator of covariant differentiation. We now have the following line 
of reasoning. If the metric is constant throughout space, then covariant differentiation reduces to ordinary differentiation in which case the former must be commutative and the commutator must vanish. Hence, the condition ( 8.14 ) for a flat manifold may be expressed as:

$$
R_{\mu \alpha \beta}^{\gamma}=0
$$

It should be noted that although we have used Christoffel symbols, we have not exploited their metric properties. Consequently, they could have been replaced throughout the preceding computation by symmetric affine symbols. Thus, our result applies to an affine as well as to the more specialized metric manifold. Furthermore, we have nowhere presupposed the Lorentz metric. Consequently, (8.19) must be regarded as purely mathematical.

In spite of the notational simplicity of (8.19), as a four-dimensional tensor of the fourth rank, it corresponds to a system of 256 second order partial differential equations. However, if we assume a metric connection, we may form the associated tensor of (8.17) by:

$$
g_{\delta \gamma} R_{\mu \alpha \beta}^{\gamma}=R \delta_{\mu \alpha \beta}
$$

which is still in the realm of pure mathematics. It is called the covariant Riemann-Christoffel tensor. By: exploiting the many symmetry properties of $R \delta \mu \alpha \beta$, it is possible to show that of the 256 components, only 20 are independent and non-vanishing.

our original motivation for constructing the 
Riemann-Christoffel tensor was to find a generally covariant field equation which admitted the Lorentz metric as a possible solution. In fact, (8.19) is sufficient but not necessary for the latter. Two points should be noted. (8.19) is the condition for a flat metric of any signature. The (L) Lorentz metric $g_{\mu v}^{(L)}$ a particular case which satisfies (8.19). However, as soon as we specify $g_{\mu v}^{(L)}$ as the particular solution of (8.19) we move from the level of mathematics to that of the interpreted object theory. The Lorentz metric is introduced for physical reasons. It is interesting to consider whether these reasons are simply to preserve the distinction between space and time or the stronger condition that the Lorentz metric is dictated by Special Relativity physics. Schrodinger has shown(op. cit., p. 85) that a global metric in which there is more than one time-like coordinate is analytically as well behaved as the standard $(3+1)$ coordinatization. Hence, we suspect that the physically interpreted counterpart of (8.19) depends on the full factual content of electrodynamics in which the Iorentz metric, as distinct from any other canonical matrix, plays a special role. For this reason, we believe that foundation workers should tackle the difficult task of axlomatizing General Relativity in such a fashion that the laws of Special Relativity would not be the basis of a correspondence principle to the effect that General Relativity must reduce to them in the degenerate 
case of gravity free space; but rather such that special Relativity physics would be a constitutive part of the higher level Theory of General Relativity.

It is clear that (8.19) is far too strong a restriction for a field equation in matter free space, since its interpreted counterpart is a law which holds globally for gravity free space. It is the precise mathematical representation of the void of Democritus. It represents a 'world completely devold of variety or content, a world of maximum uniformity. Nevertheless, it is important to us since we deliberately designed it to admit the Lorentz metric as a possible solution. Obviously, we must seek a generalization of (8.19) which satisfies our initial requirement but is sufficiently flexible to provide the basis for a covariant theory of gravitation. The next step in the formation of the covariant gravitational field equations is well known to anyone who has a passing knowledge of the Theory of General Relativity, namely the contraction of the Riemann-Christoffel tensor. However, there is some room for disagreement in regard to the basis or motivation for this essential step. It could, of course, be argued that we may ignore the matter since the heuristic criteria for the construction of a theory have no bearing on the ultimate success and acceptability of that theory in terms of its providing a basis for correct predictions, etc. However, virtually 
all writers base this step on some form of correspondence principle to the effect that the gravitational equation(s) must reduce to the Newton-Poisson equation for slow moving particles in weak fields, and since this may be achieved to a first approximation by a second order linear equation in $9_{00}$, it is assumed that the exact covariant field equation would be of second order in $g_{\mu v}$. In other words, there would be ten equations in place of the single Newton-Poisson equation, corresponding to the ten independent components of the $g_{\mathrm{mv}}$. (In fact, there can be only six such components.)

The present writer has already indicated his belief that correspondence principles are vacuous, and for this reason finds it interesting to explore metatheoretical indications for the contraction of the Riemann-Christoffel tensor. So let us pretend that we know nothing of the classical law of gravitation and see, briefly, how far metatheoretical considerations will take us. However, we emphasize that we are seeking indications and not an a prior1 derivation of Einstein's law of gravitation.

In the first place, if (8.19) were an adequate description of the field (or lack of it), say before the Creation, then the entire spirit of General Relativity would be violated, since (8.19) depends essentially on a fixed fundamental tensor gav which would take over the role of Newton's absolute space and time. Crudely speaking, 
if such a theory were possible, the gmv would play the same role in the metrical quadratic form as $\delta_{i x}$ in the theory of Newton. The geometry of space-time would be absolute although hyperbolic rather than Euclidean. In the second place, it has been shown by Hilbert(1915, Cf. also Bergmann, 1942, p. 178) that the field equations must satisfy four additional identities over and above the symmetry conditions of the curvature tensor. These correspond to the arbitrary choice of coordinates and are necessary for the attainment of a generally covariant metric. Hence, we know on formal grounds that the vanishing of the Riemann-Christoffel tensor is a necessary and sufficient condition for the manifold to be globally flat in gravity free space and that the 20 independent equations must be reduced by at least four. Furthermore, we know that (8.19) could not be a special case of the field equation in the sense, for example, in which Laplace's equation is a special case of Poisson's equation, since it is incompatible with the very idea of a principle of general relativity. It is natural, therefore, as a tentative step, to contract the Riemann-Christoffel tensor. Fortunately, there is but one such contraction that does not vanish identically, namely:

$$
R_{\mu \alpha \beta}^{\alpha}=R_{\mu \beta}
$$

which is called the contracted curvature tensor. It is a symmetric tensor with ten independent components. However, 
by virtue of the four coordinate conditions, the contraction of (8.19):

$$
R_{\mu \rho}=0
$$

is a system of six second order differential equations which are quasi-linear in the gur. It may be shown, although actually it is obvious, that (8.22) admits a flat space solution but does not require it. Thus, something like (8.22) would be a reasonable candidate for a system of gravitational equations in matter free space. We were able to arrive at $(8.22)$ by formal reasoning; but this is as far as we can go. There is no way in which metatheoretical considerations could lead us to the Lorentz metric, which must be postulated as the gravity free solution of (8.22) on physical grounds. This may seem to be very little in the way of physics and a great deal in the way of mathematics, but we repeat our conviction that the privileged role of the Lorentz metric is based on the full weight of Special Relativity physics and, in particular, on electrodynamics.

We now mention, without proof, the important mathematical result employed by Einstein. From the contracted curvature tensor, it is possible to construct another tensor of the same rank which has a vanishing divergence, which we know to be of particular significance in a space-time manifold. The Einstein or Ricci tensor is given by: 


$$
G_{\mu \nu}=R_{\mu \nu}-\frac{1}{2} g \mu \nu R^{\alpha} \alpha
$$

It was shown by Cartan(1922) that the most general second rank tensor which may be constructed wholly from the metric tensor and its derivatives is given by:

$$
B^{\alpha \gamma}=G^{\alpha \gamma}+\Lambda g^{\alpha \gamma}
$$

However, at least for the non-cosmological form of General Relativity, $\Lambda$ may be taken to be zero on physical grounds. Thus, the gravitational field equation in the absence of matter may be written as

$$
G_{\mu r}=0
$$

Finally, the more general version of (8.25) may be constructed on the assumption that the complete energymomentum tensor(See Appendix) includes every form of matter and energy apart from gravitational energy, and we may write the general law of gravitation in the form:

$$
G_{\text {Miv }}=K T_{\mu \nu} \text {. }
$$

where $\mathrm{k}$ is a universal constant. The special significance of the divergenceless character of $T^{\mu r}$ is that if any quantity is omitted from it, it would be manifested as a force and $T^{\mu r}$ could not, in that case, be divergenceless. That is to say, the total energy and momentum in the universe as represented by $T^{\mu v}$ would not be conserved even locally.

To complete our exposition of the basic laws of General Relativity, we may add a ponderomotive equation to the field equations. The equation of motion of a mass 
point moving in a gravitational field is well known to define a geodesic trajectory. This is the famous geodesic postulate which links the effects of the gravitational field to the geometry of the space-time manifold via the first and second derivatives of the metric tensor. In words, given the space-time geometry, the equation of motion may be derived from the variational principle:

$$
\delta \int d s=0
$$

In fact, however, the equation of motion may be derived as a theorem(Bunge, 1967a, p. 231; Fock, 1964, p. 240) from the condition:

$$
T^{M v}{ }_{j v}=0
$$

That is to say, the equation of motion may be derived from the field equations of which $(8.28)$ is a direct consequence. If $(8.28)$ is written out in explicit form and integrated over the volume at whose boundaries $T^{\mathrm{mr}}$ disappears, we are led by a straight computation to the ponderomotive equation of a mass point:

$$
\frac{d^{2} x^{\nu}}{d \tau^{2}}+\left\{\alpha^{\nu} \beta\right\} \frac{d x^{\alpha}}{d \tau} \frac{d x^{\beta}}{d \tau}=0
$$

As Bunge has pointed out, this does not constitute a geometrization of physics. In fact, it is neither more nor less geometrical than 'Newton I', which states that the trajectory of a free mass point is a geodesic in a Euclidean space-time manifold. This completes our brief exposition of the basic laws of General Relativity, and we are now prepared to consider its philosoph1cal implications. 
The subject of tensor analysis or manifold geometry to which the reader was given a cursory introduction is a strictly formal theory in which the variables $x^{i}$ have no physical significance. For example, the indices may run over any number $n$. A ten-dimensional differentiable manifold has the same reality, for the mathematician, as, for example, a two-dimensional Euclidean plane. However, when manifold geometry is applied to physical space-time, the situation is altogether different. In the first place, we are normally restricted to the consideration of a four-dimensional differentiable manifold. In the second place, there is a distinguished variable, $x^{0}=$ ct. Thirdly, the signature of the metric is \pm 2 , depending on convention. Fourthly, the mathematically allowable transformations must be restricted by the so-called reality conditions. From these considerations alone, it should be obvious that when it is said that the basic laws of General Relativity are generally covariant in virtue of their being expressible as tensor or pseudo-tensor equations, such a claim is misleading.

From the standpoint of the pure mathematician, a permissible transformation in Riemannian geometry is of the form:

$$
x^{M} \rightarrow \bar{x}^{\mu}=f_{\mu}\left(x^{1}, x^{2}, \ldots, x^{n}\right) \quad(\mu=1,2, \ldots n)
$$

where $f_{\mu}$ are arbitrary functions of class $C^{m}$, where $m$ may be any integer subject to the whim or design of the 
mathematician. Confronted by a tensor equation of physics, the mathematician could transform it into a form which violates the most fundamental (protophysical) requirements of any physical theory. Since, in physics, the $f_{m}$ are not arbitrary functions, it is incorrect, under any circumstances, to speak of physical laws as generally covariant, even though the geometrical objects which we employ to express those laws may, indeed, constitute a basis for the realization of a generally covariant group of transformations. Thus, at the outset, we declare that the general covariance of physical laws is a myth, one of the many engendered by the confounding of mathematical theories with the physical theories which they are employed to represent. To give a naive analogy, as every philosopher knows, there is an infinitude of sentences of English which are syntactically permissible but devoid of literal significance. Nevertheless, English syntax is still a useful system for constructing meaningful sentences. A similar relationship holds between physical laws and the mathematical formalism which is employed to express them. It should be noted that the foregoing has nothing to do with our distinction between covariance groups and relativity groups. We do not forbid time independent transformations which serve many useful computational ends. We have merely declared them to be devold of physical significance. To extend our analogy, they correspond to 
the useful but vacuous laws of logic which enable us to deduce semantically equivalent sentences from each other. In fine, what we have been saying cuts across the distinction between coordinate transformations and frame transformations, with which we shall deal later in the chapter.

The protophysical conditions, of which we have spoken, which the transformed variables must satisfy are that the $\bar{x}^{1}$ be spatial coordinates while $\bar{x}^{0}$ be temporal. Furthermore, for two points $P_{1}\left(x^{\mu}\right)$ and $P_{2}\left(x^{\mu}\right)$ : if $P_{1}\left(x^{i}\right)=$ $\mathrm{P}_{2}\left(\mathrm{x}^{1}\right)$ and $\mathrm{P}_{1}\left(\mathrm{x}^{0}\right) \neq \mathrm{P}_{2}\left(\mathrm{x}^{0}\right)$, then $\mathrm{P}_{1}\left(\overline{\mathrm{x}}^{0}\right) \neq \mathrm{P}_{2}\left(\overline{\mathrm{x}}^{0}\right)$. Moreover, the time order of $P_{1}$ and $P_{2}$ is fixed. If the time interval is infinitesimal, then the points will have temporal coordinates $\mathrm{P}_{1}\left(\mathrm{x}^{0}\right)$ and $\mathrm{P}_{2}\left(\mathrm{x}^{0}+d \mathrm{x}^{0}\right)$ and:

$$
8_{00} \mathrm{dx}^{0} \mathrm{dx}^{0}>0 \text {, }
$$

whence $\delta_{00}>0$. Similarly, given $\mathrm{P}_{1}\left(\mathrm{x}^{1}\right)$ and $\mathrm{P}_{2}\left(\mathrm{x}^{1}+\mathrm{d} \mathrm{x}^{1}\right)$ such that $P_{1}\left(x^{0}\right)=P_{2}\left(x^{0}\right)$, then:

$$
g_{1 \mathrm{k}} d \mathrm{x}^{1} d \mathrm{x}^{\mathrm{k}}<0
$$

Therefore, (8.32) must be negative definite. These conditions entail that in any infinitesimal region of $V_{4}$, there is always a permissible mapping such that $g_{\mu r}=$ diag $(1,-1,-1,-1)$ which we call the canonical form of the Lorentz metric or simply the Lorentz metric. It is also called the hyperbolic metric, since under the condition that a permissible transformation carries the entire $V_{4}$ into $\operatorname{dag}(1,-1,-1,-1)$, the geometry of the manifold has 
the so-called hyperbolic structure of Lobatchewski. (In fact, it is of some metatheoretical interest to note that by assuming a hyperbolic manifold, it is possible to express the laws of Special Relativity physics in Newtonian form - a point which is widely misinterpreied by the conventionalists) (Angel, 1961, 1962; Rougier, 1914)

Within this delimited framework, there is no question that certain types of coordinate systems play an outstanding role in General Relativity. For example, the time independent, spherically symmetrical system of Schwarzschild has served as a basis for virtually all of the computations of testable consequences of General Relativity physics. However, such outstanding coordinate systems have no direct bearing on the Principle of General Relativity, per se, but only on the confirmation of the field equations. They play a comparable role to that of spherical coordinates in computing the solution of Laplace's equation.

In general, it is impossible to discover a coordinate system for General Relativity which plays, for example, a similar role to the Cartesian system in Newtonian Mechanics; that is to say, a coordinate system which is preeminently qualified to provide a model of $a$. frame of reference. In general, in an accelerating frame of reference, the spatial geometry, not to speak of the space-time geometry, is constantly changing. Even on the 
assumption that an acceleration, per se, has no effect on geometrical measurements, a variable velocity must entail a time dependent Lorentz contraction. For these reasons, it is impossible to distinguish a privileged subset of inertial frames of reference in General Relativity. The so-called Einstein boxes or lifts are mere heuristic metaphors which are suited only to the unacceptable theory of kinematical relativity. In General Relativity, an Einstein box would enclose an infinitesimal volume and could not, therefore, be realistically regarded as a frame of reference but merely as a particle in free-fall. Bunge(1967a, p. 231) provides a definition of an inertial frame of General Relativity which is indeed consistent with the postulates of the theory. However, such frames are only vacuously realised insofar as the definition refers to static gravitational fields which do not exist in the physical world.

Nevertheless, it would be instructive to attempt to construct a privileged type of reference frame which has the appearance of being intuitively preferable to various alternatives. However, we warn the reader that our attempt will not succeed. However, the writer has discovered that in the exploration of the foundations of physics failure is often as instructive as success. We naturally take for granted the standard restrictions, already mentioned, on the four-dimensional 
differentiable space-time manifold. Let us consider a three-dimensional hypersurface $s_{3}$ of $V_{4}$ such that any vector normal to $s_{3}$ satisfies the condition:

$$
t^{\mu} t_{\mu}>0
$$

In virtue of the conditions (8.31) and (8.32), $t^{\mu}$ is $a$ time-like vector and $S_{3}$ is spatially oriented. Let $P^{*}\left(x^{i}\right)$ be a variable point in $S_{3}$. By a well-known theorem, through any $P^{*}\left(x^{i}\right)$ there is one and only one geodesic which is orthogonal to $s_{3}$. In a finite region of $s_{3}$, such geodesics will form a congruence of curves. It is clear that along any curve of the congruence, the coordinates $x^{i}$ will be fixed. The fourth coordinate of a fixed point $P$ may be given by the arc length $P^{*} P=\Delta x^{0}$. The distance along a curve of the congruence will be given by:

$$
(d s)^{2}=\left(d x^{0}\right)^{2} \text {, }
$$

whence $8_{00}=1$. Furthermore, any vector with components: $\left(0, x^{1}\right)$ must be orthogonal to the unit tangent vector $(1,0,0,0)$ at the same point, whence $\delta_{01}=g_{10}=0$. The line element on the hypersurface $s_{3}$ must, therefore, have the form:

$$
(d s)^{2}=\left(d x^{0}\right)^{2}+8_{i k} d x^{i} d x^{k}
$$

This is an intuitively satisfying metric, since it separates the spatial geometry from the time. However, we have yet to determine whether such a metric can be extended throughout the $V_{4}$. In terms familiar to the non-mathematician, it is as though we had just discovered how to coordinatize a Euclidean plane and express distances by the theorem of 
Pythagoras, and now desire to discover whether the technique may be extended to three-dimensional space. As we know, the equation of a geodesic is:

$$
\frac{d^{2} x^{\alpha}}{d s^{2}}+\left\{\begin{array}{c}
\alpha \\
\mu v
\end{array}\right\} \frac{d x^{\mu}}{d s} \frac{d x^{\nu}}{d s}=0
$$

The parameter $s$, in our case, is obviously $x^{0}$. Since the $\mathrm{d} \mathrm{x}^{i}$ are constant along the geodesic, we must have:

$$
\left.\begin{array}{l}
\left\{\begin{array}{ll}
i & i \\
0 & 0
\end{array}\right]=0 \\
{\left[\begin{array}{ll}
0 & 0, i
\end{array}\right]=0}
\end{array}\right\}
$$

Let us write the second of (8.37) explicitly:

$$
[00, i]=\frac{1}{2}\left(\frac{\partial 90 i}{\partial x^{0}}+\frac{\partial 9 i 0}{\partial x^{0}}-\frac{\partial 900}{\partial x^{l}}\right)
$$

since 800 is constant, $\frac{\partial 900}{\partial x^{i}}$ is identically zero. Hence, we deduce that:

$$
\frac{\partial 90 i}{\partial x^{0}}=0
$$

throughout at least a finite region of the $V_{4}$. That is to say, any hypersurface must be orthogonal to the timelike geodesics. Thus, their status is similar to that of the surfaces of simultaneity of Newtonian space-time. Moreover, since our manifold has a metric affinity, it follows that the time-like separations between two given hypersurfaces are comparable along all of the time-like geodesics. Thus, we have a universal, although not absolute, time parameter. We now construct a frame of reference in which the time coordinate lies along one of the time-like geodesics, which is to say that a standard clock will give a proper time reading, and the spatial axes will be oriented so that they are orthogonal to the time-like axis. 
Thus, the coordinate system with the line element (8.35) would model such a frame of reference. The reader will note that we have done the reverse of the usual procedure by inventing a system of coordinates and then hypothesizing a frame of reference which they represent.

So long as we continue to heed the requirement that $\delta_{i j} \mathrm{dx}^{i} \mathrm{dx}{ }^{j}$ be negative definite, we are free to perform any purely spatial coordinate transformations that we choose. However, we can no longer claim general covariance for the space-time metric $\delta_{\mu v} d x^{\mu} d x^{\nu}$, even in the restricted sense. Moreover, since the proper time $\mathrm{x}^{0}=c \tau$ is uniquely detiermined for different accelerating bodies, not only would the geometry of the hypersurface be relatively complex, but the transformation equations between distinct time orthogonal frames of reference would be a mathematical Chinese torture. Add to this the fact that every distinct accelerating body would require its own reference frame, and we see how fruitless our attempt has been to discover a system of coordinates which could play a preeminent role in General Relativity. Such coordinatizations, like that of schwarzschild and the harmonic coordinates favoured by Fock, are useful for obtaining solutions of specially simplified physical problems such as that of planetary motion in the gravitational field of the sun, which is treated as a one body problem. These cases are invaluable for the important 
task of testing Einstein's field equations; but while they help us to confirm the theory of gravitation, they shed no light on the broader implications of the Principle of General Relativity. By the same token, it is possible to introduce new coordinatizations of accelerating frames of reference(Cf. Whller, 1952, pp. 250ff.) which are akin to the gauge transformations of electrodynamics, such that the gravitational potentials vanish over a finite region. However, such mathematical tricks only work in the case of non-permanent fields.

Fock has argued that a privileged system of coordinates exists in the context of General Relativity, the so-called harmonic coordinates, albeit, as we shall subsequently see, he rejects the Principle of General Relativity, itself. We shall first consider the harmonic coordinates from a mathematical standpoint and then discuss their physical and methodological significance. As we know, the covariant derivative of an arbitrary contravariant vector is given by:

$$
A^{M} ; V=A^{M}, V+\left\{\begin{array}{c}
M \\
\alpha V
\end{array}\right\} A^{\alpha}
$$

Accordingly, the four-divergence of $A^{\mu}$ is given by:

$$
A^{v} ; v=A^{v}, v+\left\{\alpha^{v} v\right\} A^{\alpha}
$$

But $\{\alpha v\}=\frac{1}{2} \operatorname{gmv} \frac{\partial g^{v} \mu v}{\partial x^{\alpha}}$

is a result of standard mathematics. Moreover, if $g$ is the determinant of $8 \mu v$, then:

$$
\left\{\alpha^{v} r\right\}=\left(\frac{1}{2 g}\right) g, v=\ln (-g)^{1 / 2}, v
$$


Inserting (8.43) into (8.41), we have:

$$
A^{v}{ }^{v} v=\frac{1}{(-g)^{1 / 2}}\left[(-9)^{1 / 2} A^{v}\right], v
$$

Suppose, now, that we have a vector $A_{\mu}$ which is the gradient of a scalar $\$$. That is:

$$
A_{\mu}=\phi_{, \mu}
$$

Then,

$$
A^{V}=\operatorname{gmV} Q, M
$$

and

$$
\operatorname{Div} A^{V}=A^{V} ; V=\frac{1}{(-g)^{1 / 2}}\left[(-g)^{\frac{1}{2}} \operatorname{gMr}^{M} Q, M\right] \text { ir }
$$

which is the $d \cdot A l$ embertian of $\phi$. It should be noted that if the $x^{H}$ are four solutions of

$$
\square^{2} \phi=0
$$

then $\left\{\begin{array}{c}v \\ v\end{array}\right\}$ will vanish identically. Although this condition is seemingly trivial, the reader may verify that it leads to the non-trivial consequence that the covariant divergence will be equivalent to the ordinary divergence. In more robust terms, each axis of the coordinate system would represent a possible world line of a light ray. It is obvious that the Cartesian coordinates of special Relativity are a special case of harmonic coordinates. The reader will recall that it is necessary to supplement the $\delta_{\mu v}$ by four coordinate conditions to ensire the general covariance of the metric form. Fock's point is that instead of letting the coordinate conditions be arbitrary we make them unique by requiring them to satisfy (8.48). It could be argued that such a move would require our abandoning the requirement of general covarlance. However, this would not be a fair criticism, since Fock does not argue for the 
mathematical necessity of harmonic coordinates but for their privileged physical role. Naturally, if we restrict the expression of physical laws to special systems of coordinates, such expressions cease to be generally covariant; but this in no way precludes our returning to the use of arbitrary coordinates if we should so desire.

It is well known to the student of electrodynamics that the equation for the propagation of an actromagnetic wave front is a special case of electromagnetic propagation which corresponds to a discontinuity of the field. Obviously, the surfaces on which the field is discontinuous must coincide with the advancing wave fronts. such surfaces, in electrodynamics, are known as the characteristic surfaces of Maxwell's equations. Expressing the wave front equation implicitly in the form:

$$
\omega(x, y, z, t)=0
$$

we express the law of wave propagation in vacuo in the form:

$$
\frac{1}{c^{2}}\left(\frac{\partial \omega}{\partial t}\right)^{2}-(\nabla \omega)^{2}=0
$$

which is the equation of a characteristic of Maxwell's laws. By the same token, Fock(1964, p. 194) is able to show that employing harmonic coordinates and expressing the equation of a gravitational wave front implicitly as:

$$
\omega\left(x^{0}, x^{1}, x^{2}, x^{3}\right)=\text { const. }
$$

one is able to express the law of gravitational propagation in the form:

$$
\operatorname{gmv} \frac{\partial \omega}{\partial x^{\mu}} \frac{\partial \omega}{\partial x^{\nu}}=0
$$


which is in conformity with the speciel relativistic requirement of the existence of a limiting velocity. It should be clear that Fock's introduction of harmonic coordinates as essentially privileged is quite contrary to the point of view of General Relativity, which is not to say that such systems have no practical value. It is noteworthy that in the application of such coordinates to physical problems, one is restricted to the case of insular mass distributions (one body problems) and particular boundary conditions, the most important of which is that the gravitational waves die off at infinity, so that the metric must assume the Lorentz form over the boundary surface of the 'universe'. We may interpret this condition either as an example of physically untested, and probably untestable, cosmological speculation or as an artificial simplification designed for the purpose of achieving computational results, i.e. solutions of the field equations à la schwarzschild. Physically, the harmonic coordinates are possible models of unaccelerating frames containing or constituting a very large insular mass. Fock argues that one of the principal methodological advantages of such models is that they distinguish permanent from impermanent gravitational fields. From our point of view, this is a philosophical disadvantage since it forces the reintroduction of the concept of fictitious force which is unsuited to a satisfactory theory of the real, 
i.e. non-fictitious, world. A methodologically sound theory should have no recourse to fictions of any kind, with the possible exception of purely mathematical ones. In fine, we regard Fock's viewpoint as a rather Procrustean one, which endeavours to squeeze the theory of gravitation into the restricted framework of special Relativity, while missing the entire point of the Principle of General Relativity which does not require the conjuring up of a 'universe' which obviously does not correspond to reality. It is unfortunate that a man of Fock's intellectual stature, attested to by several important insights such as the rejection of operationalism, should fall prey to one of the common vices of the physicist, namely that of attempting to justify a set of special assumptions which facilitate the solving of equations by destroying an entire theory that honestly endeavours to represent the nature of physical reality. A position similar to our own, but less harsh, is taken by Bunge in his axiomatization of the Theory of General Relativity. Commenting on the harmonic coordinates, he writes:

\footnotetext{
"... though particular coordinate systems should be irrelevant at the level of principles, they become conspicuous at the level of theorems, both for the latter's statement and for their physical interpretation. But it does not show that Nature wears one kind of coordinates preferably to others." (1967a, p. 230. The italics are ours.)
} 
In fine, we conclude that it is impossible to find a distinguished subset of physically realizable frames of reference which play the role analogous to that of the inertial frames associated with a restricted principle of relativity. In this regard, the adjective general is well chosen to describe Einstein's principle of relativity. But, of course, liberty is not to be confused with license, which, in this case, means that General Relativity is not to be interpreted as General Covariance. It is true that the field equations are generally covariant. But that does not imply that we may take advantage of that formal property to introduce coordinate systems which model physically unrealizable frames of reference. We wish to underline, however, that such formal manipulations are nevertheless permissible on the basis of the structure of the theory of tensors. Hence, while the Principle of General Relativity does not distinguish a subset of inertial frames of reference, it does distinguish a subset of physically permissible space-time transformatiors. Moreover, the frames of reference which are physically attainable do form an equivalence class of a kind, although, as one should expect, not of the same kind as that which we associate with restricted principles of relativity.

We may define a physically realizable spatial frame of reference as one which, while not necessarily rigid, which is, strictly speaking, excluded even by the 
Theory of special Relativity, is such that no point of reference may have a velocity greater than the velocity of light with respect to any other point of reference belonging to the same frame. Thus, the tangential velocity of a distant star may exceed the velocity of light with respect to the rotating earth but the earth and the star do not belong to the same frame of reference. A second condition for a physically realizable frame of reference, which may not be independent of the first, is that it is a frame with respect to which the time track of a light ray is a null geodesic. Since the time track is null in a Lorentz frame, a condition which may be expressed by:

$$
(d \tau)^{2}=g_{\mu \nu} d x^{\mu} d x^{2}=0
$$

and since $(8.56)$ is a tensor equation, the time track must be null in every permissible frame of reference. These conditions may easily be shown to be equivalent to the following definition of a physically permissible frame of reference.

A physically permissible frame of reference is one which may be so coordinatized that for every infinitesimal region of the manifold, there exists a permissible mapping such that the metric coeficients may be transformed to $g_{M v}^{(c)}=\operatorname{diag}(1,-1,-1,-1)$. That the metric of any Riemannian manifold may be transformed to sylvester canonical form by a non-singular transformation is a formal condition that follows from the theory of symmetrical 
matrices. That the canonical form in question be $g_{m}^{(c)}$ is not a peculiarity of Riemannian manifolds but of the physical space-time manifold. We shall call the class of reference frames which satisfy this special property of the manifold Einstein frames. Any theory which can be expressed in a form, not necessarily by means of the tensor calculus, that is indifferent to the set of Einstein frames will be termed generally relativistic.

En passant, we are now in the position to understand the deeper reason, to which we alluded earlier, for the incompatibility of Classical and special Relativity. The canonical form of the gmv in an infinitesimal region of the space-time manifold, i.e. the signature of the metric, is a peculiarity of the given manifold which is either imposed arbitrarily or on the basis on physical considerations. While the mapping which transforms the metric to canonical form is not unique, the canonical form, Itself, is. The signature of the usual classical(GalileoNewton) space-time metric is $(0,1,1,1)$. Thus, the incontrovertible reason for the impossibility of a Galilei covariant Nawtonian Mechanics and a Lorentz covariant electrodynamics is that it would entail that the spacetime manifold simultaneously carry two different metric signatures, a mathematical impossibility. The present writer has no idea whether this has previously been pointed out but finds it curious that it is not mentioned in any 
of the standard treatises with which he is familiar. We may now speak more precisely of the relationship between General Relativity and special Relativity. It would be simplistic to argue, as some writers do, that General Relativity is simply a generalization of special Relativity, despite its being the probabie motivation for Einstein's entertaining the idea of General Relativity in the first place. It is equally wrong to suggest that Lorentz frames in the form of so-called Einstein boxes constitute the equivalence class of inertial frames of General Relativity. Such frames only occur in the special, and non-existent, case of the field of an infinitely extended sheet of matter of uniform density. They belong to Einstein's speculations during the perlod between the special and General theories in which he thought of a general theory of relativity as a kinematical generalization of Special Relativity on the basis of the Principle of Equivalence. It is well known that such heuristic reasoning led to qualitative predictions which were most remarkable but, nevertheless, Inaccurate to the order of as much as $50 \%$.

Conversely, we should consider the status of Special Relativity physics in the light of the General Theory. Altnough Special Relativity supplied General Relativity with an essential ingredient, namely $g_{\mathrm{mv}}^{(\mathrm{h})}$, we are bound to admit that the laws of special Relativity 
physics must now be treated as approximations, al though their approximate character may doubtless be ignored in the realm of strong interactions. To take the two basic theories which we have considered in earlier chapters, namely point mechanics and electrodynamics, their respective positions $\underline{v i s}$ à $\underline{\text { vis }}$ General Relativity are not absolutely identical. We would suggest that whereas Special Relativity Mechanics is strongly incompatible with General Relativity, Special Relativity Electrodynamics is only weakly incompatible with it. A special relativistic theory rests on the assumption of a flat metric in finite, if not global, regions of the space-time marifold. This, in turn, entails the absence of permanent gravitational fields. Now, since material bodies are sources of the gravitational field, Special Relativity Mechanics is rendered inconsistent in the light of General Relativity. In the case of electrodynamics the situation may be somewhat different. Bunge has argued(1967a, pp. 200ff.) that the celebrated $' E=m c^{2}$, is a theorem of special Relativity liechanics, and should not be interpreted as implying that radiant energy has inertia. In that case, it is conceivable that electromagnetic fields are not $\theta+\infty$ $\therefore$ isints Shetein $\because 2 \tan _{2}+2$ $\sin \sin$ sources of gravitational fields, in which case the matter tensor should be appropriately modified. In that case, one could conceive of a unlverse populated only by electromagnetic flelds which produce no gravitational

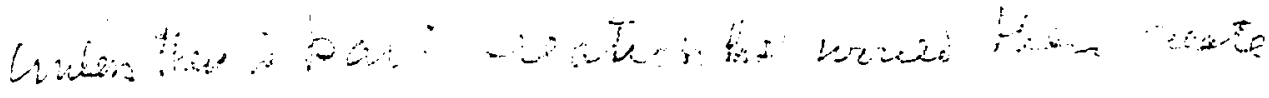

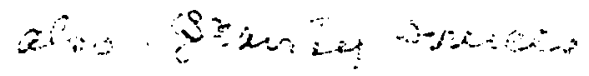

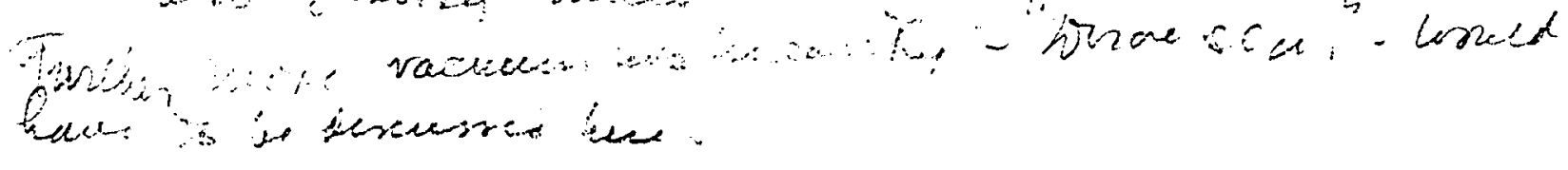


effects, and which obey Maxwell's laws rigorously in the large. In that case, electrodynamics would be a logically consistent theory, rigorously compatible with the field equation:

$$
G_{\mu v}=0
$$

Thus, special relativistic electrodynamics could be the basis for a consistent description of a non-existent universe. Special relativistic mechanics, on the other hand, is strictly incompatible with General Felativity. We hope that our scientific readers will forgive the foregoing speculative remarks which, in any case, play no essential role in this dissertation. We made them only as a suggestion concerning one of the many matters to be taken into account in what we regard as the still open foundation problem concerning the relationship between Special Relativity and General Relativity. Moreover, it rests on the shoulders of the experimental physicist to determine definitively whether the electromagnetic field $\therefore$ is indeed a gravitational source.

Let us now deal with Fock's criticism of the Einsteinian interpretation of the Principle of General Relativity. Fock correctly argues that general covariance is a formal principle which holds that any putative law of nature must be independent of the coordinatization. He treats it, therefore, as a correct consistency requirement on the basic laws of physics but as nothing more. 
Furthermore, following Cartan(1927), he argues for the vacuity of the general covariance of the metrical quadratic form on the ground that one has complete freedom in the choice of the transformed coefficients $\bar{g}_{\mu \nu}$. The reader will recali that given a function $f$ of a certain argument $A$, the function is covariant under a given group of transformations if and only if the transformed function of the transformed argument $\bar{f}(\bar{A})$ is of the same form as the original. This is indeed the case for the laws of Newtonian Mechanics under the Galilean group and for the laws of electrodynamics under the Lorentz group. For example, when we employ the imaginary coordinate ict, the Lorentz metric has the form $\delta_{\mu v} d x^{\mu} d x^{\nu}$, which under an

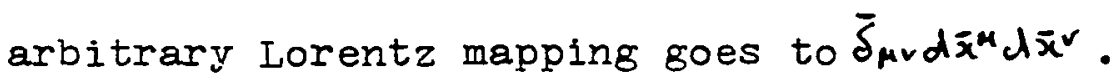

Moreover, Fock argues that the existence of an equivalence class of frames of reference, namely inertial frames, is directly related to the degree of uniformity of the manifold. Specifically, in an n-dimensional manifold, the maximum uniformity of a geometrical object is attained when that object is mapped onto itself under a group of $\frac{1}{2} n(n+1)$ independent parameters. For example, the surface of a sphere is a two-dimensional manifold, and the largest group of transformations which map every point of the spherical surface onto points of the same surface, 1.e. map the sphere onto itself, is a three-parameter group. Similarly, the space-time manifold is four-dimensional. 
Therefore, the most general group which maps the manifold onto itself must be a ten-parameter group, which is precisely the order of the Lorentz group. Accordingly, Fock argues, it is impossible to generalize on the transformation theory of Special Relativity which employs a manifold of maximum uniformity. In particular, the expression 'General Relativity' is, according to Fock, a contradictio in adjecto. Einstein's second theory should not be called the Theory of General Relativity, but simply the theory of gravitation. (It is presumably for this reason that Fock sees so much significance in the harmonic coordinates.)

Let us begin by pointing out that we are in accord with Fock on at least one point, namely that one should not be seduced by the highly compressed notation of the tensor formalism. The mapping:

$$
g_{\mu v} d x^{\mu} d x^{v}=\bar{g}_{\mu v} d \bar{x}^{\mu} d \bar{x}^{v}
$$

certainly appears to be covariant. However, whether it is truly covariant can only be determined when the ten mappings represented by (8.58) have been expressed in explicit form. We have already remarked that the formal notion of covariance is ambiguous to the extent to which it depends on the notation which happens to be employed. The practical value of the tensor formalism is that it enables one to write equations which are independent of any particular system of coordinates. We have seen, in fact, 
that even in the case of Galilean and Lorentz transformations, the question as to whether the covariantly transformed laws have the same physical content as the original expressions requires careful physical analysis. on the other hand, Fock's argument is really rather trivial. On the basis of Fock's premisses, Einstiein is beaten before he begins. The requirement that the transformed function of its transformed argument be isomorphic with the original function of the original argument could only be satisfied under a group of linear transformations and, hence, only by a restricted principle of relativity. If we took fock seriously, we would be taking undue liberties in carrying out so elementary a transformation as that from Cartesian to spherical coordinates, which is non-linear. We would violate physical principles in expressing the divergence and curl operators of elementary vector analysis in spherical coordinates. But, as every physicist surely knows, it would be possible to expound the entire compass of special Relativity physics in terms of curvilinear coordinates without altering the content of the theory in any way. In fact, we prefer Cartesian coordinates precisely because they permit the Lorentz transformation to be expressed as a linear transformation.

Actually, Fock has fallen into the very trap which he set for Einstein, whom he accuses of falling to 
understand his own theory. On the one hand, he argues that the Principle of General Covariance is a purely formal requirement which any theory can be made to satisfy and indeed, for logical reasons, should satisfy, while a principle of relativity is a physical matter; but, on the other hand, he claims that there can be no principle of general relativity on the basis of a purely formal criterion. In fact, whether or not there is a principle of general relativity, that is to say whether or not all physically realizable frames of reference are equivalent is a matter which can be settled only be experiment. This is the case for restricted and general principles alike. We are justified in claiming that Maxwell's equations satisfy the Principle of Special Relativity not because it is possible to write them in manifestly Lorentz covariant form, but are justified in writing them in such a form because it has been experimentally determined that they satisfy the Principle of Special Relativity. Crudely speaking: if several scientists performed appropriate experiments in distinct Lorentz frames, they would all agree that the results of their respective experiments were in conformity with the predictions of Maxwell's laws of electrodynamics. Then they would find it convenient to employ the four-vector formalism which is indifferent with respect to Lorentz frames. By the same token, if several scientists performed 
appropriate experiments, either mechanical or optical, in distinct Einstein frames, i.e. physically realizable but otherwise arbitrary frames, and found that in all cases the gravitational effects were compatible with Einstein's field equations, they would be justified in claiming that Einstein's laws were in conformity with the Principle of General Relativity. Then they could claim a physical justification for writing those laws in generally covarlant form which, among other things, is indifferent with respect to Einstein frames. It is unfortunate, of course, that the tensor formalism is indifferent to any frame including those which are physically unrealizable.

of course, the excessive latitude of the tensor formalism may be remedied simply by stating the necessary restrictions on the $g_{\mu v}$. However, It would be a useful task for the applied mathematician to attempt to axiomatize a restricted theory of space-time tensors whose formal structure would be based on the local Lorentz character of the manifold. They would be geometrical objects whose covariance group corresponded to the general relativity group. We might call such objects either steinors or framors. The first step would be to work out the theory of representations of the group which maps Einstein frames into Einstein frames. The rest should be easy. (our assumption that such a group exists rests on physical 
intuition and epistemological considerations.) Such a suggestion, incidentally, would have no significance for Newtonian Mechanics in which three-vectors are quite adequate geometrical objects. It is true that we make a distinction between the relativity group and the covariance group of Newtonian liechanics. However, apart from time reversal, the transformations which are excluded from the relativity (sub)group are only so excluded on the bas is of their triviality. In the case of General Relativity, the situation is different. Transformations exist which are mathematically permissible, in terms of the formalism, but must be excluded from the relativity group on the ground of their physical impossibility. If we may be permitted briefly to psychologize, we would suggest that Fock, like many other physicists, has been seduced into regarding the Iinear relationships between inertial frames as given a priori, merely because the two known principles of relativity, apart from the General Principle, happen to pertain to frames of reference that are linearly related. However, there is nothing self-contradictory in the concept of an accelerating inertial frame unless, I1ke Fock, one chooses to define such frames out of existence. We repeat our earlier implication that Fock's argument is specious because it is irrefutable and no physical hypotheses are irrefutable. In a nutshell, Fock rejects the Principle of General 
Relativity on the ground that it contravenes a requirement that only a restricted principle of relativity could possibly satisfy.

While we contend that the Principle of General Relativity is a genuine physical hypothesis, we are bound to admit that its experimental support is very slight and only indirect. We spoke hypothetically of performing experiments in distinct Einstein frames. Unfortunately, the only significant case of an Einstein frame in which we may perform experiments is the solar system. It would be pointless to attempt to test Einstein's field theory in a man-made Einstein frame such as an accelerating rocket, since all of the experimental results would be in conformity with the classical law of gravitation as well as with the Einsteinian law. It would seem that a satisfactory: test of the Principle of General Relativity must await the journey by some future Astronomer Royal to another star system.

On the other hand, there is considerable presumptive evidence in support of the Principle of General Relativity insofar as the generall: covariant fleld equations, albeit in the special Schwarzschild case, have met with remarkable predictive success. We refer, of course, to the red-shift, the precession of Mercury, and the deflection of starlight. It is worthwhile to remark at this point on the distinction between the experimental 
confirmation of a theory and the experimental confirmation of a relativity principle which that theory putatively satisfies. This further supports our contention that a principle of relativity is not a metalaw but an independent physical hypothesis. No physical hypothesis is capable of absolute verification, but a principle of relativity is, in principle, as testable as any other object statement of science.

It is interesting to compare the points of view of Bunge and Fock vis à vis General Relativity. According to Fock, such a principle is physically impossible since it necessarily fails to conform to certain formal requirements of the space-time manifold. In other words, he rejects what he regards as a physical hypothesis on certain formal grounds, which we have held to be appropriate only to restricted principles of relativity. On the other hand, he accepts the gravitational field equations, while treating their general covariance as a mere consistency requirement that every fundamental law must satisfy. Bunge, in contrast, does not reject the Principle of General Relativity but incorrectly identifies it with the Principle of General Covariance, thus relegating it to the metatheoretical level.

Let us now state our own position in capsule form to contrast it with those of Bunge and Fock. Firstiy, we hold that it is essential to distinguish between the 
relativity group of a theory and its covariance group. The Principle of General Relativity belongs to a different level of scientific discourse from that of the Principle of General Covariance. It is obvious that the verification of the general covariance of Einstein's field equations is, as Bunge would argue, a paper and pencil operation. The testing of the Principle of General Relativity, on the other hand, requires observation and experiment in at least two distinct Einstein frames. Finally, in any case, no physical theory could strictly satisfy the Principle of General Covariance, even in the formal sense, since every physical theory must satisfy the reality conditions which are imposed on the $g_{\mathrm{mv}}$ but which are not part of the formal structure of tensor theory. For example, per impossibile, if the velocity of electromagnetic propagation were infinite, then the equations of electrodynamics would be Galilel covariant. But this would in no way preclude their being written in generally covariant form, i.e. as tensor equations. Equivalently, the demonstrations by Cartan and others that classical mechanics may be written in generally covariant form should not be interpreted as trivializing the Principle of General Relativity (à la Kretschmann) but as confirming its status as an object law rather than as a metalaw. In fine, we must distinguish between the form of general covariance and the substance of general relativity. 
As our grand finale, we offer our formal

statement of the Principle of General Relativity.

$\{I\}$ is the set of fundamental laws of a given theory.

$\{K\}$ is the set of physically realizable frames of

reference, i.e. Einstein frames.

$\{T\}$ is the set of mathematically admissible coordinate transformations.

For any $I \in\{L\}$, any $t \in\{T\}$ and any $k_{i}, k_{j} \in\{K\}$ :

I w.r.t. $k_{i} \in\{K\} \leftrightarrow t(I)=s$. $\bar{l}$ w.r.t. $k_{j} \in\{K\}$.

The reader is urged immediately to compare the

foregoing with the formal statement of the Principle of

Special Relativity. He should note the following fundamental

distinctions between the two. $\{T\}$ is here restricted

only by the weakest formal requirements on coordinate transformations in general. On the other hand: the denotation of $\{K\}$ places definite limits on the $t_{1} \in\{T\}$ which would, in fact, be applicable. The generality of the above principle is reflected in its ranging over the entire $\{K\}$ as distinct from a distinguished subset of the latter, which was the case for both of the restricted principles. Finally, it should be noted that the latitude in the interpretation of $\{I\}$ reflects our view that the Theory of General Relativity is not merely a theory of gravitation but rather one in which gravitational laws necessarily play a fundamental role. 


\section{APPENDIX TO CHAPTER VII}

This appendix merely serves to add some physical information to Chapter VII. The mathematical machinery is simply that which is required for special Relativity physics. However, our purpose is to construct a new tensorial quantity which plays an essential role in the Theory of General Relativity. The following treatment is purely physical, and absolutely no philosophical considerations will arise. For details, the reader should consult a textbook on relativity physics. What follows is simply a sketch which is adequate for the purposes of this dissertation. Its only virtue is its brevity. Given a continuous distribution of incoherent

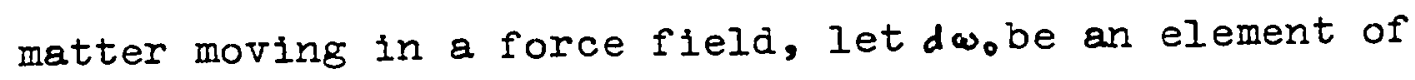
the proper volume of the mass. We grite the defining equation:

$$
F^{M}=d f \cdot D^{M} d \omega_{0}
$$

We call $D^{\mu}$ the four-force density. In component form, it may be written as:

$$
D^{M}=(\vec{d}, i / c \vec{d} \cdot \vec{v})
$$

Now we consider the case of a supposedly continuous charge distribution given by $J^{M}$. If d $\omega_{0}$ is an element of proper volume of the charge distribution and $\rho_{0}$ is the proper charge density, then by (7.46) and (7.65), we have:

$$
F^{M}=\rho_{0} / C F^{M V} U^{V} d \omega_{0}=\frac{1}{c} F^{M V} J^{V} d \omega_{0}
$$


Hence, by (9.1), we may define the four-force density of the electromagnetic field by:

$$
D^{M}=\frac{1}{c} F^{M V} J^{v}
$$

Substituting the first of (7.58) in (9.4) gives:

$$
D^{M}=\frac{1}{4 \pi} F^{M V} F^{\sigma V}, \sigma
$$

We now wish to show that $(9.5)$ is the negative of the divergence of a particular symmetric tensor which we call the energy-momentum tensor of the electromagnetic field. We define the latter tensor by:

$$
S^{\mu v}=\frac{1}{4 \pi} F^{\mu \sigma} F^{v \sigma}-\frac{1}{16 \pi} \delta_{\mu v} F^{\sigma \rho} F^{\sigma \rho}
$$

Taking the divergence of (9.6). We have:

$$
S^{\mu v}, v=\frac{1}{4 \pi} F^{\mu \sigma}, F^{v \sigma}+\frac{1}{4 \pi} F^{\mu \sigma} F^{v \sigma_{1 V}}-\frac{1}{8 \pi} \delta_{\mu v} F^{\sigma} P F^{\sigma} \sigma_{, V} \text { (9.7) }
$$

By virtue of the antisymmetry of $F^{A v}$ and the second equation of (7.58), a few algebraic manipulations will yield:

$$
S^{M V}, V=\frac{1}{4 \pi} F^{M \sigma} F^{V \sigma}, V=-\frac{1}{4 \pi} F^{M \sigma} F \sigma_{, V}=-D^{M}
$$

The reader may ascertain the physical significance of $S^{\mu V}$ by substituting the values of $F^{M V}$ from the matrix array (7.57a). We merely mention the more significant results. $S^{i j}$ is the negative of Maxwell's stress tensor, which by virtue of the suppression of $s^{14}$ and $s^{41}$ is only a tensor in an inertial frame with a fixed coordinatization. $s^{i 4}=s^{4 i}$ is Poynting's vector $\vec{s}$, multiplied by a factor $1 / c$. Finally,

$$
S^{44}=-\frac{1}{8 \pi}\left(|\vec{E}|^{2}+|\vec{B}|^{2}\right)=-U
$$

which is the energy density of the electromagnetic fleld. 
In summary :

$$
S^{\mu v}=\left(\begin{array}{c|c}
t^{i j} & \vec{S} / i_{c} \\
\hline \vec{S} / i c & U
\end{array}\right)
$$

We have, thus far, ignored the mass of the point charges. However, if we assume a system of point charges which are not interacting mechanically, by virtue of the constancy of their proper mass, one may easily write an equation of continuity for proper mass. That is to say, if $S$ is a closed surface bounding a volume $V$, then the rate at which proper mass is flowing through $S$ must be equal to the decrease of proper mass in $V$. If da is an element of volume of the charge distribution with respect to $K$, we may write the proper mass of the element as $\mu_{0} d \omega$. We know this quantity to be Lorentz invariant. But dw is not an invariant, hence $\mu_{0}$ cannot be an invariant. It is actually the density of proper mass with respect to $K$. We may write our equation of continulty in the form:

$$
-\frac{d}{d t} \int_{v} \mu_{0} d \omega=\int_{s} \mu_{0} \vec{v} \cdot d \vec{s}
$$

By Gauss's theorem, the right hand side of (9.11) is given by:

$$
\int_{\vec{s}} \mu_{0} \vec{v} \cdot d \vec{s}=\int_{v} \nabla \cdot M_{0} \vec{v} d \omega
$$

By (9.11) and (9.12), we have:

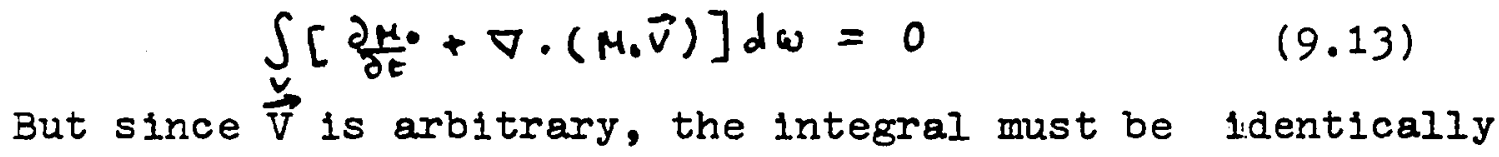
zero; whence:

$$
\frac{\partial \mu_{0}}{\partial t}+\nabla \cdot \mu_{0} \nabla=0
$$


Let $\mu_{0}$ be the proper density of the proper mass. Then:

$$
\mu_{00} d \omega_{0}=M_{0} d \omega
$$

Clearly, $\mu_{0}$ must be Lorentz invariant. Hence, by (9.15):

$$
\mu_{00}=\left(1-v^{2} / c^{2}\right)^{1 / 2} \mu_{0}
$$

Therefore, the equation of continuity may be written in manifestly covariant form as:

$$
\mu_{00} U^{\mu}, M=0
$$

The reader should compare (9.17) with (7.44). By (9.1), the equation of motion may be formed as:

$$
D^{M}=\mu_{00} \frac{d U r}{d \tau}
$$

Furthermore, by virtue of $(9.17)$ and an easy computation, it may be shown that:

$$
\left(\mu \circ U^{v} U^{\mu}\right)_{\nu \mu}=D^{M}
$$

We now define a symmetric tensor $\theta^{\mu v}$ by:

$$
\theta^{M V}=d f \text {. Moo } U^{M} U^{v}
$$

Whence:

$$
D^{H}=\theta^{M v}, v
$$

We may leave it to the reader to work out the significance of the components of $\theta^{\mu v}$, known as the kinetic energymomentum tensor, on the basis of its being the counterpart for matter of the energy-momentum tensor $S^{\mu s}$. By (9.8) and $(9.21)$, it is obvious that:

$$
-S^{\mu V}, r=\theta^{M v}, V
$$

It should be emphasized that (9.22) is not an identity but a physical hypothesis. Finally, we construct the tensor: 


$$
T^{M v}=S^{M v}+\theta^{M r}
$$

By (9.22), it follows that:

$$
T^{M v}, r=0
$$

$T^{\mu \nu}$ is the complete energy-momentum tensor which comprises the energy contributions of both the electromagnetic field and matter.

We have crammed into a single quantity, albeit on the basis of several simplifications, every type of energy contribution except gravity. (We, of course, forget about nuclear forces.) (9.24) at least has the appearance of a continuity equation. Sometimes $T^{\mu \nu}$ is called the matter tensor. In a covariant theory of gravitation, we should expect $\mathrm{T}^{\mathrm{\mu \nu}}$, or something analogous, to play the role of the source of the gravitational field.

We apologize for the terseness of this appendix, which was really just an interlude between special Relativity and General Relativity. At least, the lay reader will now have a limited conception of the physics of General Relativity which is treated in a limited way in Chapter VIII, which is of a far different order from. having the ability to follow its mathematical formalism. 


\section{BIBLIOGRAPHY}

A complete bibliography, broadly interpreted, would have required tens of thousands of entries. Narrowly interpreted, a complete bibliography would have required no more than about twenty entries. The following is a compromise consisting of those works which have had some effect, not necessarily positive, on this dissertation.

Adler, R., M. Bazin, H. Schiffer. Introduction to General Relativity. New York, McGraw Hill, 1965 .

Anderson, J.I. Principles of Relativity Physics. New York, Academic Press, 1967.

Angel, R.B. Henri Poincaré's Theory of Conventionalism. Unpublished M.A. thesis, McGill University MS., 1961.

Angel, R.B. "Poincaré and Reichenbach on the Relativity of Physical Space," in Thought, Toronto,W.J. Gage, 1962.

Bateman, H. The Transformation of the Electrodynamical

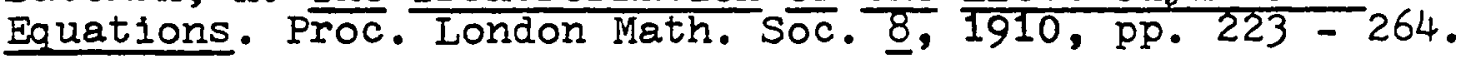
Bazin, M. See Adler, R. et al.

Bergmann, P.G. Introduction to the Theory of Relativity. Englewood Cliff, Prentice-Hall, 1942.

Bergmann, P.G. "The Special Theory of Relativity," Handbuch der Physik, IV, Berlin,Springer Verlag, 1962, pp. $109-202$. Bergmann, P.G. "The General Theory of Relativity," ditto, pp. $203-272$.

Boya, L.J. A Note on Time-Reversal Invariance. Am. J. Phys., 33, 1965, pp. 139ff.

Bridgman, P.W. A Sophisticates Primer of Relativity. Connecticut, Harper, 1965. (Reprint of Wesleyan U.P. edition, 1962) 
Broad, C.D. Scientific Thought. New Jersey, Littlefield, Adams, 1959. (Reprint of Routledge and Kegan Paul edition, 1923.)

Bunge, M. Metascientific Queries. Springfield, N.J., Ch. C. Thomas, 1959.

Bunge, M. Laws of Physical Laws. Am. J. Phys., 29, 1961, pp. $518-\overline{529}$.

Bunge, M. The Myth of Simplicity. Englewood Cliffs, N.J., Prentice-Hall, 1963.

Bunge, M. Foundations of Physics. Springer Tracts in

Natural Philosophy, v. 10, New York, Springer Verlag, 1967a

Bunge, M. Scientiflc Research. (2 vols.) New York, Springer Verlag, $1967 \mathrm{~b}$.

Cartan, E. Sur les équations de la gravitation d'Einstein. J. Math. pur. et appl. 1, 1922, $\overline{\mathrm{pp}} \cdot \frac{141-203 .}{141}$

Cartan, E. Sur les variétés à connexion affine et la théorie de la relativité gériẻralisée. Ann. Ecole Norm. 40, 1923, pp. $3 \overline{25}=412 ; 41,1924, \mathrm{pp} \cdot 1-25 ; 42,1925, \mathrm{pp}$. 17-28.

Cartan, E. La théorie des groupes et la géométrie.

L'enseign. Math.,26, $1 \overline{927}, \frac{\mathrm{pp} .200}{2} \mathrm{22} 5$.

Cartan, E. La cinématique newtonienne et la théorie des espaces réglées à connexion euclidienne. Ass. France Avencem. Sci., $193 \overline{3, \mathrm{pp} .19}=20$.

Dewitt, B.S. "Dynamical Theory of Groups and Fields," in Relativity, Groups and Topology. (edd. C. Dewitt and B. Dewitt) New York, Gordon and Breach, 1964, pp. 587 - 820 .

Dicke, R.H. Gravitation without a Principle of Equivalence. Rev. Mod. Phys., 29, 1957, pp. $363-376$.

Dicke, R.H. The Theoretical Significance of Experimental Relativity. See Dewitt(1964)

Eddington, A. The Philosophy of Physical Science. Cambridge U.P., 1939.

Einstein, A. 'On the Electrodynamics of Moving Bodies," in The Principle of Relativity. See Einstein et al., 1923.(1905)

Einstein, A. "On the Influence of Gravitation on the Propagation of Iight," ditto. (1911) 
Einstein, A. "The Foundation of the General Theory of Relativity," ditto. (1916)

Einstein, A. Prinzipielles zur allgemeinen Relativitatstheorie. Ann. der Phys., 55, 1918, pp. $241-244$.

Einstein, A. The Meaning of Relativity. (5th. edition), Princeton U.P. 1954. (First edition, 1922)

Einstein, A., H.A. Lorentz, H. Minkowski, H. Weyl. The Principle of Relativity. New York, Dover, n.d. (Reprint of viethuen edition, 1923.)

Einstein, A. "Autobiographical Notes," See Schilpp(1949).

Eisenhart, I.P. Continuous Groups of Transformations: New York, Dover, 1961. (Reprint of Princeton U.P. edition, 1933)

Fock, V. Three Lectures on Relativity Theory. Rev. Mod. Phys. 29, 1957 , pp. $325-333$.

Fock, V. The Theory of Space, Time and Gravitation. (2nd. edition) $\overline{\mathrm{New}}$ York, Pergamon, 1964.

Fourès-Bruhat, Y. See Lichnerowicz(1962).

Goldstein, H. Classical Mechanics. Mass. Addison-Wesley, 1950

Granbaum, A. "Conventionalism in Geometry," in The Axiomatic Method.(edd. I. Henkin, P. Suppes, A. Tarski), Amsterdam, North-Holland, 1959.

Grunbaum, A. Philosophical Problems of Space and Time. New York,Knopf, 1963.

Gupta, S.N. Einstein's and other Theories of Gravitation. Rev. Mod. Phys. 29, 1957, pp. 334-336.

Havas, P. "General Relativity and the Special Relativistic Equations of Motion of Point Particles," in Recent Developments in General Relativity. New York Mackillan, 1962.

Havas, P. "Foundations Problems in General Relativity," in Delaware Seminar in the Foundations of Physics. (ed. M. Bunge),

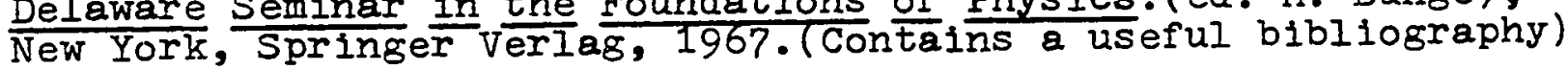

Hesse, M.B. Forces and Fields. New Jersey, Littlefield, Adams, 1965. (Reprint of Philosophical Library edition, 1961.) 
Higman, B. Applied Group-Theoretic and Matrix Methods. oxford U.P., 1955.

Hilbert, D. Die Grundlagen der Physik(I). Nachr. kgl. Ges. Wiss. Gottingen, 15,1915 , pp. 395 - 407 .

Hill, E.L. On the kinematics of uniformly accelerated motions and classical electromagnetic theory. Phys. Rev., 72, $1947, \mathrm{pp} .143-149$.

Hill, E.L. The deformation of moving coordinate systems in relativistic theories. phys. Rev., 84, 1951, pp. 1165-1168.

Infeld, L. and A. Schild. On the Motion of Test Particles

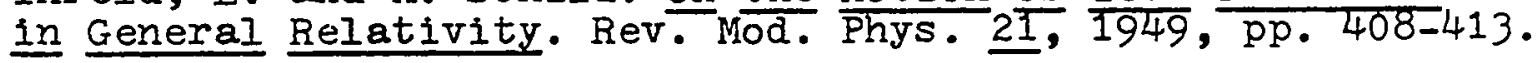

Infeld, L. Equations of Motion in General Relativity Theory and the Action Principle. Rev. Mod. Phys., 29, 1957, pp. $398-411$.

Klein, 0. "Mach's Principle and Cosmology in Relation to General Relativity." See Havas (1962)

Kretschmann, $E$. ther den physikalischen sinn der Relitivitutspostulate. Ann. der Phys. 53, 1917, pp. $575-614$.

Kursunoglu, B. Correspondence in the Generalized Theory of Gravitation. Rev. Mod. Phys. 29, 1957, pp. 412 - 416.

Lawden, D.F. An Introduction to Tensor Calculus and Relativity. London, Methuen, 1962.

Lemaltre, G. Cosmological Application of Relativity.

Rev. Mod. Phys., 21, 1949, pp. 357-366.

Lichnerowicz, A. and Y. Fourès-Bruhat. "Problèmes Mathématiques en Relativité." See Havas(1962)

Loedel, E. Geometric Representation of the Lorentz Transformation. Am. J. Phys., 25, 1957, pp. 327ff.

Margulies, G. Remark on Kinematically Preferred Co-ordinate Systems. Proc. Nat. Acad. Sc1. U.S.A., 42, 1956, pp. 1521.

McKinsey, J.C.C. and P. Suppes. Transformations of systems of classicle particle mechanics. J. Rat. Mech., $\underline{\underline{2}}$, 1953; pp. $\frac{91933-289 .}{273}$

Minkowsk1, H."Space and Time." See Einstein et al.(1923) (1908) 
Møller, C. The Theory of Relativity. Oxford, Clarendon Press, 1952 .

Murnaghan, F.D. The Theory of Group Representations: New York, Dover, 1963. (Reprint of Johns Hopkins edition, 1938)

Noonan. T.W. See Robertson(1968).

Pathria, R.K. On Transformations in Relativistic Mechanics. Am. J. Phys., 24, 1956, pp. 411ff.

Post, E.J. Formal Structure of Electromagnetics. Amsterdam, North-Holland, 1962.

Post, E.J. "General Covariance in Electromagnetism," See Havas (1967).

Prokhovnik, S.J. The Logic of Special Relativity. Cambridge U.P., $1 \overline{967}$.

Purcell, E.M. Electricity and Magnetism. New York, McGrawHill, 1963.

Robb, A. The Absolute Relations of Time and Space. Cambridge U.P., 1941.

Robertson, H.P. Transformations of Einstein Spaces. Proc. Nat. Acad. Sci. U.S.A., 11, 1925, pp. $590-592$.

Robertson, H.P. Dynamical Space-Times winich Contain a Conformal Euclidean 3-Space. Trans. Amer. Math. Soc., 29, 1927, pp. 481 - 496.

Robertson, H.P. Groups of Motions in Spaces Admitting

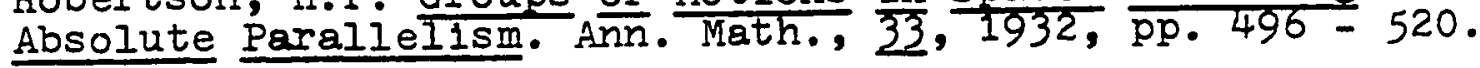
Robertson, H.P. "Geometry as a Branch of Physics," See Schilpp(1949)

Robertson, H.P. Postulate Versus observation in the Special Theory of Relativity. Rev. Mod. Phys., 1949 a, 21, pp. $378=382$.

Robertson; H.P. and T.W. Noonan. Relat1V1ty and Cosmology. Toronto, W.B. Saunders, 1968.

Rosen, N. A Farticle at Rest in a Static Gravitational Field. Rev. Mod. Phys., 21, 1949, pp. 503-505. 
Rosen, N. and H. Shamir. Gravitational Field of an Axially Symmetric System in First Approximation. Rev. Mod. Phys., 29, 1957, pp. $429-431$.

Rosenthal-Schneider, I. "Presuppositions and Anticipations in Einstein's Physic," in Schilpp(1949).

Rougier, I. De I'utilisation des Géométries non-euclidiennes dans la physique de la relativité. I'Enseign. Math, 16, $1914, \overline{p p} \cdot 5-18$.

Schiffer, M. See Adler et al. (1965).

Schild. A. See Infeld(1949).

Schilpp, P.A. (ed.) Albert Einstein: Philosopher-scientist. Library of Iiving Philosophers, Evanston, Tudor, 1949.

Schlegel, R. Time and the Physical World. New York, Dover, 1968. (Reprint of Michigan U.P. edition, 1961.)

Schrolinger, E. Space-Time Structure. Cambridge U.P., 1950.

Schwartz, H.M. Introduction to Special Relativity.

Toronto, McGraw-Hili, 1968.

Shamir, H. See Rosen(1957).

Souria, J.M. Géométrie et relativité. Paris, Hermann, 1964.

Stoll, R.R. Set Theory and Logic. San Francisco,

W.H. Freeman, 1961.

Suppes, P. See Mckinsey(1953).

Synge, J.I. Relativity: The General Theory. Amsterdam, North-Holland, 1960 .

Synge, J.L. Introduction to General Relativity. See Dewitt(1964).

Synge, J.L. Relativity: The Special Theory.(2nd. edition) Amsterdam, North-Holland, 1965.

Thomas, L.H. The Kinematics of an Electron With an Axis. Phil. Mag., 3, 1927.

Thomas, T.Y. Kinematically preferred co-ordinate systems. Proc. Nat. Acad. Sc1. U.S.A., 41, 1955, pp. $762-770$. 
Trautman, A. "Foundations and Current Problems of General Relativity," in Lectures on General Relativity. New Jersey, Prentice-Hall, $1 \overline{965 .}$

Van Dantzig, D. The fundamental equations of electromagnetism: independent of metrical geometry. Proc. Cambridge Phil. Soc., 30, 1934, pp. $4 \overline{21-427}$.

Van Dantzig, D. Über das Verhältnis von Geometrie und Physik. c. r. Congr. Int. Math. oslo(1936), $\underline{2}, 1937, \mathrm{pp} .2 \overline{25}-\frac{227}{2}$

Veblen, 0 . Invariants of quadratic differential forms. Cambr. tracts math. and math. phys., No 24, Cambridge, 1927.

Weatherburn, C.E. An Introduction to Riemannian Geometry and the Tensor Calculus. Cambridge U. P., 1938.

Weinstock, R. Derivation of the Lorentz-Transformation Equations Without a Iinearity Assumption. Am. J. Phys., 32, 1964, pp. $260 \mathrm{f}$.

Weyl, H. Space-Time-Matter. (4th. edition). New York, Dover, 1950 . (Reprint of 1921 edition).

Weyl, H. The Classical Groups, their Invariants and Representations, Princeton U.P., 1946.

Whitrow, G.J. The Fitzgerald-Lorentz contraction phenomenon and theories of relativity of Galilean frames.

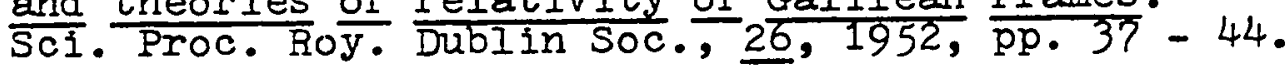

Whitrow, G.J. The Natural Philosophy of Time. New York, Harper, 1963. (Reprint of Thomas Nelson edition, 1961.) 


\section{Errata}

The author is indebted to Dr. David Salt of McGill University, whose careful reading of this dissertation uncovered the following errors and mental lapses.

p. 19: In fact, the group operation is not defined for every pair of elements. Hence, the set of mathematically admissible coorainate transformations is the realization of a groupoid or pseudo group but not of a group.

p. 62: The status of mass as a scalar object is merely sufficient but not necessary for the covariance of Newton's first law.

p. 62: Delete constant from the penultimate line.

p. 79: (4.30) is generally covariant. It is the equation of an affine geodesic.

p. 82: The second paragraph is incorrect. Time translations do not involve time reversal.

p. 88, line 13: $f, 8, j, h$ are, of course, linear functions not constant functions.

pp. 161 \& 190: The reality conditions imposed on the $g_{i k}$ are only required on the assumption that a reference frame is of the standard type having three spatially oriented axes and one time-like axis. However, the utilisation of a different kind of reference frame would not have any effect on the metric signature of the manifold.

p. 197: The sentence which begins on Iine 14 is incorrect and should be deleted. 